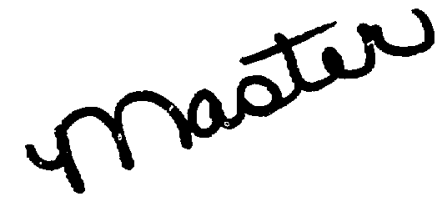

\title{
SOLA-STAR:
}

\section{A One-Dimensional}

\section{ICED-ALE Hydrodynamics Program for Spherically Symmetric Flows}

\author{
L. D. Cloutman
}

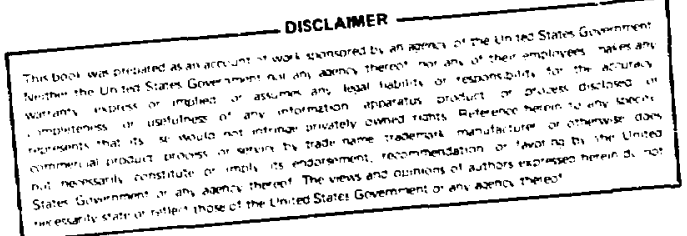


SOLA-STAR: A ONE-DIMENSIONAI, ICED-ALE HYDRODYNAMICS

PROGKAM FOR SPHERICALLY SYMMETRIC FLOWS

by

L. D. Cloutman

\begin{abstract}
This report describes a simple, generalpurpose, and efficient algorithm for solving one-dimensional spherically symmetric, transient fluid-dynamics problems using a variation of the ICED-ALE technique. Included are the finite difference equations, three test problems that illustrate various capabilities of the program, and a complete code description, including a listing, sample data decks and output, a summary of important variable names, and hints for conversion to other operating systems.
\end{abstract}

\title{
I. INTRODUCTION
}

Several. years ago we reported a technique for implementing the ICED-ALE methodology in a form sulcable for numerically simulating a wide variety of spherically symmetric fluid flows. ' An experimental computer progran, VEGA, vas written to test this methodology and was applied to the star formation problem. Although that technique was designed for astrophysical applications, it is by no mearis limited to them. In the interim, Group $T-3$ has developed the SOLA series of simplified numerical fluid dynamics programs ${ }^{2,3}$ speciflclally for public distribution. In response to requests for copies of the VEGA program, we are presenting a simplified version, SOLA-STAR, in this report. This code follows the phijosophy of the SOLA series inasmuch as the code is easy to understand and use, it can be used by persons with little numerical fluid dynamics experience, it is easily modif led to include more complicated physics, and it is useful both as a teaching device and a serious research tool. 
The numerical algorithm used in the present program is basically the same as reported in Ref. 1, so no derivation of the difference equations will be presented in this report. The derivation is based on a volume integration of the governing equations, and the interested reader can find the details in Refs. I and 4-6. The algorithm consists of two phases. Phase I is a partially implicit Lagrangian time step. In Phase II, the solution is rezoned (if desired) in a physically motivated manner that conserves mass, momentum, and internal energy. The only stability requirement is that

$$
\frac{|\mathbf{u}| \delta t}{\delta \mathbf{r}}<1
$$

everywhere on the mesh, where $u$ is the velocity, $\delta t$ is the time step, and $\delta r$ is the width of a computational mesh cell. This 1 imit requires that the fluid moves less than one cell width each computational cycle. Observance of this $1 \mathrm{imit,}$ proper choice of the donor cell parameter $\alpha$ (to be described later), and choosing the time step such that no variable changes its value by more than some small amount, say $20 \%$, has been sufficient to achieve numerical stability for all of the problems that we have run. A more detailed discussion of stability of the method (and of many other aspects of the method) can be found in Ref. 1 .

\section{EQLATIONS FOR SPHERICALLY SYMMETRIC FLOWS}

To simplify the program and minimize both computing time and core requirements, SOLA-STAR assumes a single-component ideal gas. The differential equations that we model are

$$
\begin{aligned}
\frac{\partial \rho}{\partial t}+\frac{1}{r^{2}} \frac{\partial}{\partial r}\left(r^{2} \rho u\right) & =0, \\
\frac{\partial \rho u}{\partial t}+\frac{1}{r^{2}} \frac{\partial}{\partial r}\left(r^{2} \rho u^{2}\right) & =-\rho g-\frac{\partial p}{\partial r}+\frac{1}{r^{2}} \frac{\partial}{\partial r}\left(r^{2}(2 \mu+\lambda) \frac{\partial u}{\partial r}\right)-\frac{2 u(2 \mu+\lambda)}{r^{2}} \\
& +\frac{2 u}{r} \frac{\partial \lambda}{\partial r},
\end{aligned}
$$


and

$$
\begin{aligned}
\frac{\partial \rho I}{\partial t}+\frac{1}{r^{2}} \frac{\partial}{\partial r}\left(r^{2} \rho u I\right) & =-p \frac{1}{r^{2}} \frac{\partial}{\partial r}\left(r^{2} u\right)+\frac{1}{r^{2}} \frac{\partial}{\partial r}\left(r^{2} K \frac{\partial T}{\partial r}\right)+2 \mu\left[\left(\frac{\partial u}{\partial r}\right)^{2}\right. \\
& \left.+\frac{2 u^{2}}{r^{2}}\right]+\lambda\left[\frac{1}{2} \frac{\partial}{\partial r}\left(r^{2} u\right)\right]^{2},
\end{aligned}
$$

where $t$ is time, $r$ is radius, $\rho$ is the density, $u$ is the radial velocity, $p$ is the pressure, $\mu$ is the coefficient of viscosity, $\lambda$ is the second coefficient of viscosity, $g$ is the gravitational acceleration, $I$ is the specific internal energy, $K$ is the conductivity, and $T$ is the temperature. Normally we use

$$
\lambda=-\frac{2}{3} \mu,
$$

which is accurate for an ideal monatomic gas. If experimental values of $\lambda$ are available for polyatomic gases, they can be used. However, the program will need modification. The graviational acceleration is computed from a difference approximation to

$$
g=\frac{4 \pi G}{r^{2}} \int_{0}^{r} \rho x^{2} d x
$$

where $G$ is the gravitational constant. This procedure is more accurate than solution of the Poisson equation for the gravitational potential. The set of equations is closed by the equation of state, which we assume to be

$$
p=(\gamma-1) \rho I,
$$

where $\gamma$ is the rat io of specific heats. 


\section{DIFFERENCE EQUATIONS}

The SOLA-STAR difference equations are written in terms of the primitive variables $p, \rho, I, r$, and $u$. Furthermore, simple averages are used to $f$ ind values of variables at points other than those where they are defined. Transformations of variables are frequently advocated as a means of achieving better accuracy on a given computational mesh. However, as discussed in the SOLA-ICE report, ${ }^{3}$ this approach has a number if disadvantages and pitfalls for generalpurpose programs. First, it is much easier to create conservative difference schemes in the primitive variables. Second, transformations commonly introduce transcendental functions such as square roots and exponentials into the equations, and these functions are expensive to compute. Third, the transformed equations are usually more complicated, resulting in more debugging effort and increased execution $t$ ime. Fourth, the transformation that gives the best accuracy is problem dependent and, in general, unknown. Heuristic arguments that lead to particular transformations are at best unreliable. Finally, if good resolution is used, all well-behaved transformations will give the same results as the primitive variables.

Advancement of the variables in time is accomplished in two phases. Phase I consists of a partially implicit Lagrangian time step, and Phase II consists of the rezoning procedure. The velocity is defined at cell edges, (or vertices) as shown in Fig. 1, and all other quantities are defined at cell centers.

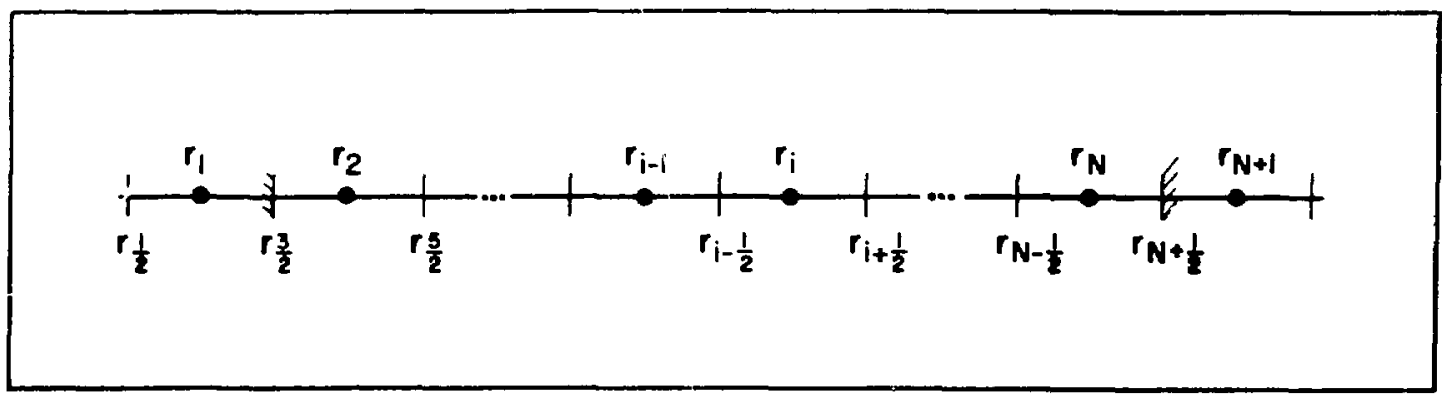

FIg. 1 .

The SOLA-STAR computing mesh. Cell centers have integer subscripts, and cell edges have half-integer subscripts. We specify the cell edge positions and define the cell center positions by $r_{i}=0.5 *\left(r_{i+\frac{1}{2}}+r_{i-\frac{1}{2}}\right)$. Ceils 1 and $N+1$ are
fictitious cells. 
The spatial difference approximations for Phase I are derived by integrating the dynamical equations over a control volume taken to be a spherical shell coincident with the computational mesh. This procedure has been adequately described elsewhere, $1,4,5,6$ so it will not be repeated here. The equations are written as fully implicit, and then they are made 1 inear in the advanced time quantities. The 1 inearization is illustrated by the equation of state:

$$
p_{j}^{n+1}=(\gamma-1)_{j}^{n+1} \rho_{j}^{n+1} I_{j}^{n+1} \doteq(\gamma-1)_{j}^{n}\left(\rho_{j}^{n+1} I_{j}^{n}+\rho_{j}^{n} I_{j}^{n+1}-\rho_{j}^{n} I_{j}^{n}\right)
$$

where the superscript denotes the time level, and the subscript denotes the spatial computational cell. We have computed protostellar models through central hydrogen dissociation and ionization (where the $\gamma$ of the gas changes radically) with no sign of instability from the use of the explicit value of $\gamma$. In such a case we define $\gamma$ not as the ratio of specific heats, but as $\gamma-1 \equiv$ P/ II. The function $\gamma-1$ is constant over much of the $(\rho, T)$ plane, so a bilinear interpolation is accurate. In the regions of the $(\rho, T)$ plane where $(\gamma-1)$ is not constant, this interpolation scheme may be preferred over many of the higher order schemes advocated in the literature, including both second and third order polynomials and splines. Indiscriminant use of some of these schemes can introduce spurious oscillations into $\gamma-1$, leading to inaccurate numerical solutions.

Let us write the continuity equation as

$$
\frac{d \rho}{d t}+\rho D=0,
$$

where

$$
D=\frac{1}{r^{2}} \frac{\partial}{\partial r}\left(r^{2} u\right)
$$


Define the quantity

$$
\cdot d_{j}^{n}=-\frac{\delta t}{1+\delta t D_{j}^{n}}
$$

where

$$
D_{j}^{n}=\frac{u_{j+\frac{1}{2}}^{n}-u_{j-\frac{1}{2}}^{n}}{r_{j+\frac{1}{2}}-r_{j-\frac{1}{2}}}+2 \frac{u_{j+\frac{1}{2}}^{n}+u_{j-\frac{1}{2}}^{n}}{r_{j+\frac{1}{2}}+r_{j-\frac{1}{2}}}
$$

Then Eq. (9) is approximated by

$$
\rho_{j}^{n+1}-d_{j}^{n} \rho_{j}^{n} D_{j}^{n+1}=\rho_{j}^{n},
$$

which may be expanded to

$$
\begin{aligned}
& \rho_{j}^{n+1}-u_{j+\frac{1}{2}}^{n+1} \rho_{j}^{n} d_{j}^{n}\left(\frac{2}{r_{j+\frac{3}{2}}+r_{j-\frac{1}{2}}}+\frac{1}{r_{j+\frac{1}{2}}-r_{j-\frac{3}{2}}}\right)-u_{j-\frac{1}{2}}^{n+1} \rho_{j}^{n} d_{j}^{n}\left(\frac{2}{r_{j+\frac{1}{2}}+r_{j-\frac{3}{2}}}\right. \\
& \left.\quad-\frac{1}{r_{j+\frac{1}{2}}-r_{j-\frac{1}{2}}}\right)=\rho_{j}^{n} .
\end{aligned}
$$

As with all our difference equations, the geometric quantities (that is, $r, A$, and $V$ ) are the old-time values. The left-hand side of Eq. (9) is differenced directly because the control volume integration procedure would provide no immediate information on new densities. It would merely give us the trivial fact that the mass in a cell does not change. The advanced-time cell volume, necessary to compute the advanced time density from the cell mass, is not directly avallable. 
The equation for the specific internal energy may be written as

$$
\frac{\partial \rho I}{\partial t}+\frac{1}{r^{2}} \frac{\partial}{\partial r}\left(r^{2} \rho u I\right)=-p D+\frac{1}{r^{2}} \frac{\partial}{\partial r}\left(r^{2} k \frac{\partial T}{\partial r}\right)+\Phi .
$$

For numerical reasons we have found it expedient to define the flux

$$
F=K \frac{\partial T}{\partial r}=K \frac{\partial}{\partial r}\left(\frac{I}{c_{v}}\right)
$$

and carry along this extra equation. For normal gases, $K=\mu c_{p} / P r$, where $\operatorname{Pr}$ is the Prandtl number and $c_{p}$ is the specific heat at constant pressure. For stellar problems where we are modeling radiation diffusion, $K$ is the radiative conductivity. The quantity $c_{v}$ is defined as $I / T$, so it is not always the usual specific heat. It is tabulated and treated numerically the same as $\gamma-1$ for the general case. Incidentally, the turbulent conductivity defined by Eq. (28) of Ref. 1 did not work well and was replaced by an estimate based on the mixing length theory. The diffusion term in Eq. (15) is replaced by

$$
\frac{1}{r^{2}} \frac{\partial}{\partial r}\left(r^{2} F\right)
$$

Carrying the additional flux equation is necessary wherever $\chi \equiv$ $\log _{10}\left(K \delta t / \rho c_{v} \delta r^{2}\right)$ approaches or exceeds the number of digits carried in the calculation, because the coefficient matrix has a term like $1+2 \times 10^{X}$. The one gets lost in round off if $X$ is too large, and the matrix package cannot successfully recover the one in the course of solving the linear system.

The expression for the viscous dissipation term in $\mathrm{Eq}$. (15) is 


$$
\Phi \equiv 2 \mu\left[\left(\frac{\partial u}{\partial r}\right)^{2}+\frac{2 u^{2}}{r^{2}}\right]+\lambda\left[\frac{1}{r^{2}} \frac{\partial}{\partial r}\left(r^{2} u\right)\right]^{2}
$$

The difference approximation to Eq. (15) is derived by integrating over the spherical shell between $r=r_{j-\frac{1}{2}}$ and $r=r_{j+\frac{1}{2}}$, using the procedure described in Ref. 1. Define normalized cells volumes

$$
v_{c, j} \equiv r_{j+\frac{1}{2}}^{3}-r_{j-\frac{1}{2}}^{3}
$$

and normalized vertex areas

$$
A_{j-\frac{1}{2}}=3 r_{j-\frac{1}{2}}^{2}
$$

Then

$$
\begin{aligned}
I_{j}^{n+1} & =I_{j}^{n}+\delta t\left\{\frac{V_{c, j}}{M_{c, j}} \Phi_{j}+\frac{1}{M_{c, j}}\left[A_{j+\frac{1}{2}} F_{j+\frac{1}{2}}^{n+1}-A_{j-\frac{1}{2}} F_{j-\frac{1}{2}}^{n+1}\right]\right. \\
& -\frac{1}{2 M_{c, j}}\left[P_{j}^{n+1}\left(A_{j+\frac{1}{2}} u_{j+\frac{1}{2}}^{n}-A_{j-\frac{3}{2}} u_{j-\frac{1}{2}}^{n}\right)+p_{j}^{n}\left(A_{j+\frac{1}{2}} u_{j+\frac{1}{2}}^{n+1}\right.\right. \\
& \left.\left.\left.-A_{j-\frac{1}{2}} u_{j-\frac{1}{2}}^{n+1}\right)\right]\right\}
\end{aligned}
$$

where 


$$
M_{c, j}^{n}=\rho_{j}^{n} v_{c, j},
$$

and

$$
\begin{aligned}
\Phi_{j} & =u_{j}{ }^{n}\left\{2\left[2\left(u_{j+\frac{1}{2}}^{n+1}-u_{j-\frac{1}{2}}^{n+1}\right)\left(u_{j+\frac{1}{2}}^{n}-u_{j-\frac{1}{2}}^{n}\right)-\left(u_{j+\frac{1}{2}}^{n}-u_{j-\frac{1}{2}}^{n}\right)^{2}\right]\left(r_{j+\frac{1}{2}}-r_{j-\frac{1}{2}}\right)^{-2}\right. \\
& +2\left[4\left(u_{j+\frac{1}{2}}^{n+1}+u_{j-\frac{1}{2}}^{n+1}\right)\left(u_{j+\frac{1}{2}}^{n}+u_{j-\frac{1}{2}}^{n}\right)-2\left(u_{j+\frac{1}{2}}^{n}+u_{j-\frac{1}{2}}^{n}\right)^{2}\right]\left(r_{j+\frac{1}{2}}+r_{j-\frac{1}{2}}\right)^{-2} \\
& -\frac{2}{3\left(v_{c, j}\right)^{2}}\left[2\left(A_{j+\frac{1}{2}} u_{j+\frac{1}{2}}^{n+1}-A_{j-\frac{1}{2}} u_{j-\frac{1}{2}}^{n+1}\right)\left(A_{j+\frac{1}{2}} u_{j+\frac{1}{2}}^{n}-A_{j-\frac{1}{2}} u_{j-\frac{1}{2}}^{n}\right)\right. \\
& \left.\left.-\left(A_{j+\frac{1}{2}} u_{j+\frac{1}{2}}^{n}-A_{j-\frac{1}{2}} u_{j-\frac{1}{2}}^{n}\right)^{2}\right]\right\} .
\end{aligned}
$$

The $v$ iscous dissipation term is positive-definite if all velocities are at the same time level, but we lose this physical characteristic by using velocities from a mixture of time levels. This is probably not serious, but it should be noted.

The flux equation is

$$
F_{j+\frac{1}{2}}^{n+1}=\frac{2 K_{j+\frac{1}{2}}^{n}}{r_{j+2 / 3}-r_{j-\frac{1}{2}}}\left(\frac{I_{j+1}^{n+1}}{c_{v, j+1}^{n}}-\frac{I_{j}^{n+1}}{c_{v, j}^{n}}\right) .
$$

The momentum equation is given by

$$
\begin{aligned}
\frac{\partial \rho u}{\partial t}+\frac{1}{\mathbf{r}^{2}} \frac{\partial}{\partial \mathbf{r}}\left(\mathbf{r}^{2} \rho \mathbf{u}^{2}\right)= & -\rho g-\frac{\partial p}{\partial \mathbf{r}}+\frac{1}{\mathbf{r}^{2}} \frac{\partial}{\partial \mathbf{r}}\left[\mathbf{r}^{2}(2 \mu+\lambda) \frac{\partial u}{\partial \mathbf{r}}\right] \\
& -\frac{2 u(2 \mu+\lambda)}{\mathbf{r}^{2}}+\frac{2 u}{\mathbf{r}} \frac{\partial \lambda}{\partial \mathbf{r}} .
\end{aligned}
$$


The Lagrangian form of Eq. (24) is differenced by integrating over a spherical shell (momentum control volume) between $r_{j-1}=0.5\left(r_{j-3 / 2}+r_{j-\frac{1}{2}}\right.$ ) and $r_{j}=$ $0.5\left(r_{j+\frac{1}{2}}+r_{j-\frac{1}{2}}\right)$.

$$
\begin{aligned}
& \frac{M_{, j-\frac{1}{2}}^{n}}{\delta_{t}}\left(u_{j-\frac{1}{2}}^{n+1}-u_{j-\frac{1}{2}}^{n}\right)=-M_{v, j-\frac{1}{2}}^{n} g_{j-\frac{1}{2}}-2 v_{v, j-\frac{1}{2}} \frac{\left(p_{j}^{n+1}-p_{j-1}^{n+1}\right.}{r_{j+\frac{1}{2}}-r_{j-3 / 2}} \\
& +\frac{4}{3}\left[A_{j} u_{j}^{n} \frac{u_{j+\frac{1}{2}}^{n+1}-u_{j-\frac{1}{3}}^{n+1}}{r_{j+\frac{1}{2}}-r_{j-\frac{1}{2}}}\right. \\
& \left.-A_{j-1} \mu_{j-1}^{n} \frac{u_{j-\frac{1}{2}}^{n+1}-u_{j-3 / 2}^{n+1}}{r_{j-\frac{1}{2}}-r_{j-3 / 2}}\right] \\
& -\frac{4}{3} v_{v, j-\frac{1}{2}}\left[\frac { u _ { j - \frac { 1 } { 2 } } ^ { n + 1 } } { r _ { j - \frac { 1 } { 2 } } } 2 \left(\frac{\mu_{j}^{n}-\mu_{j-1}^{n}}{r_{j+\frac{1}{2}}-r_{j-3 / 2}}\right.\right. \\
& \left.\left.+\frac{\mu_{i}^{n}+\mu_{j-1}^{n}}{r_{j-\frac{1}{2}}}\right)\right] \text {, }
\end{aligned}
$$

where the vertex masses and vertex volumes are given by

$$
\begin{aligned}
& M_{v, j-\frac{1}{2}}=\left(M_{c, j}+M_{c, j-1}\right) / 2, \\
& v_{v, j-\frac{1}{2}}=r_{j}^{3}-r_{j-1}^{3} .
\end{aligned}
$$

The shell areas, $A_{j}$, are defined at cell centers, as indicated by the integral subscripts. To obtain the gravitational acceleration, we perform the sum

$$
g_{j-\frac{l}{2}}=\frac{4 \pi G}{3 r_{j-\frac{1}{2}}} \sum_{i=2}^{j-1} M_{c, i} .
$$


The equations form a banded IInear system in the advanced time quantities, so they may be solved by a banded matrix package, such as the one by Hindmarsh. The left element of each row of the band is stored in the computer with an index of 1. A simple mnemonic display of the subscripting scheme is given in the program listing in Appendix A.

In Phase II we are modeling the convection term

$$
\iint_{S} \rho Q\left(\underline{u}_{g}-\underset{\sim}{\tilde{u}}\right) \cdot \hat{n} d s,
$$

where ${\underset{\sim}{\mathrm{u}}}_{\mathrm{g}}$ is the grid velocity and $\underset{\sim}{\tilde{\mathrm{C}}}$ is the fluid velocity at the end of Phase I. We define the difference velocity for our one-dimensional problems, $w_{j-\frac{1}{2}} \equiv u_{g, j-\frac{1}{2}}-\widetilde{u}_{j-\frac{1}{2}}$, which is the velocity of the mesh relative to the fluid. Then $w_{j-\frac{1}{2}} A_{j-\frac{1}{2}} \delta t$ is the volume relative to the fluid that is swept out by the moving grid point. One might be tempted to take for, say the density, simply an average of the densities on either side of the moving mesh point. This is called centered differencing, and it is unstable. For this reason we use a mixture of centered differencing and donor cell differencing. The donor cell component adds a strong stabilizing diffusional truncation error that compensates for the destabilizing diffusional error of centered differencing.

Define the donor cell parameter, $\alpha_{j+\frac{1}{2}}$, by

$$
\alpha_{j+\frac{1}{2}}=-\bar{\alpha} \operatorname{sgn}\left(w_{j+\frac{1}{2}}\right)
$$

where the funstion sgn is the sign of the argument, and $\bar{\alpha}$ is a constant, $0 \leqslant \bar{\alpha} \leqslant 1$. As an example of the difference form of the convection term for a cell centered quantity,

$$
\begin{aligned}
M_{c, j}^{n+1} & =M_{c, j}^{n}-\frac{\delta t}{2}\left\{w_{j-\frac{1}{2}} A_{j-\frac{1}{2}}\left[\left(1+\alpha_{j-\frac{1}{2}}\right) \tilde{\rho}_{j-1}+\left(1-\alpha_{j-\frac{1}{2}}\right) \tilde{\rho}_{j}\right]\right. \\
& \left.-w_{j+\frac{1}{2}} A_{j+\frac{1}{2}}\left[\left(1+\alpha_{j+\frac{1}{2}}\right) \tilde{\rho}_{j}+\left(1-\alpha_{j+\frac{1}{2}}\right) \tilde{\rho}_{j+1}\right]\right\}
\end{aligned}
$$


The tildes denote results from Phase $I$. This is a straightforward approximation to Eq. (29) for $Q=1$. The density is obtained by calculating volumes from the new mesh position

$$
\mathbf{r}_{j-\frac{1}{2}}^{n+1}=\mathbf{r}_{j-\frac{1}{2}}^{n}+u_{g, j-\frac{1}{2}} \delta t
$$

Then

$$
\rho_{j}^{n+1}=\frac{M_{c, j}^{n+1}}{v_{c, j}^{n+1}},
$$

which ensures mass cunservation. The convection of internal energy is handled in exactly the same manner.

For morentum the controi volume runs from cell center to cell center, and a slight modification is necessary. The difference veloctty mist be obtained by averaging the difference velocities of the neighboring vertices. This leads to

$$
\begin{aligned}
& u_{j-\frac{1}{2}}^{n+1}=\frac{1}{M_{v, j-\frac{1}{2}}^{n+1}} \int_{v, j-\frac{1}{2}} u_{j-\frac{1}{2}}^{n}-\frac{\delta t}{4}\left[\tilde { \rho } _ { j - 1 } ( w _ { j - \frac { 1 } { 2 } } + w _ { j - 3 / 2 } ) A _ { j - 1 } \left(\left(1+\alpha_{j-1}\right) \tilde{u}_{j-3 / 2}\right.\right. \\
& \left.\quad+\left(1-\alpha_{j-1}\right) \tilde{u}_{j-\frac{1}{2}}\right)-\widetilde{\rho}_{j}\left(w_{j-\frac{1}{2}}+w_{j+\frac{1}{2}}\right) A_{j}\left(\left(1+\alpha_{j}\right) \tilde{u}_{j-\frac{1}{2}}\right. \\
& \left.\left.\left.\quad+\left(1-\alpha_{j}\right) \widetilde{u}_{j+\frac{1}{2}}\right)\right]\right\}
\end{aligned}
$$

where

$$
\alpha_{j}=-\bar{\alpha} \operatorname{sgn}\left(w_{j-\frac{1}{2}}+w_{j+\frac{1}{2}}\right)
$$

It is not necessary to use the same $\bar{\alpha}$ in the momentum eçiation as in the equation for the mass or energy. We have found empirically that ve need more donor cell in the mass and energy equations to keep cells from emptying out in the neighborhood of steep gradients. 
For problems with strong shocks, an explicit artificial viscous pressure is helpful in attaining numerical stability and accurate jump conditions. The form we have chosen is

$$
q_{j}^{n}=-\Lambda p_{j}^{n}\left(x_{j+\frac{1}{2}}-x_{j-\frac{1}{2}}\right)^{2} D_{j}^{n} \min \left(0, D_{j}^{n}\right)
$$

where $\Lambda$ is a constant of order unity. To the right side of Eq. (20), we add - it $q_{j}^{n} D_{j}^{n}$. To the right side of Eq. (25), we add $2 v_{v, j-\frac{1}{2}}\left(q_{j-1}^{n}-q_{j}^{n}\right) /\left(r_{j+\frac{I}{2}}-\right.$ $\left.r_{j-3 / 2}\right)$. In regions of expansion, $q$ vanishes. In regions of compression, the $q$ terms provide velocity diffusion in the momentum equation and "viscous" conversion of kinetic energy to thermal energy in the $I$ equation. These terms have an effective kinematic viscosity that is roughly the fluid velocity times a mesh cell size in tie neighborhood of a shock. The artificial viscous effects are concentrated in the regions of strongest compression, precisely where they are needed the most. For problems with no shocks, $\Lambda=0$ is recommended.

IV. NUMERICAL EXAMPLES

This section contains three numerical examples that illustrate the kinds of problems that may be solved with SOLA-STAR. The first example provides a test problem to be used to check out new copies of the code. These examples ar zrude simulations of physical problems, and are not intended to be compared to observations without some refinement. The first problem is the early collapse phase of a protostellar cloud. This is hasically the same problem solved by Larson. 8 The second test problem is a simple blast wave for which there is an analytical solution. The third problen is the solar wind solution by Hundhausen and Gentry. ${ }^{9}$ They considered the effects of transients imposed on a steady state solar wind.

A. Collapse of a Protostellar Cloud

The first numerical fluid dynamics calculation of the collapse of a dense interstellar cloud to form a protostar was publsshed by Larson. 8 His initial condition was an isothermal cloud of uniform density that was just unstable toward gravitational col1.jpse according to the Jeans criterion. The outer boundary condition was $u=0$ at just under the Jeans' radius. Larson's solution for a one solar mass cloud was confirmed by Ruppel and Cloutman, ${ }^{1}$ and the results 
presented in this subsection and the code listing and output in Appendix $A$ are for a similar one solar mass cloud.

Since some results of VEGA calculations were described fully and compared to Larson's results in reference 1, we will 1 imit the present discussion to the use of this problem as a test case for new copies of the program. Appendix A provides the actual computer output at $0,1,500$, and 3000 cycles. The following physical events can be seen in the solution as it develops. First, a rarefaction is created at the outer boundary at $t=0$ by the collapse of the cloud. It travels inward at the speed of sound. The density is spatially constant but temporally increasing inside the rarefaction, and it falls off as $1 / \mathrm{r}^{2}$ outside. 'This behavior is illustrated in figure 2 with the curve from cycle 300 $\left(t=2.981 \times 10^{12} \mathrm{~s}\right)$. The velocity profile consists of two 1 inear segments with the minimum at the rarefaction, as illustrated in figure 3 . The material is isothermal at $10 \mathrm{~K}$. When the rarefaction reaches the center, the embryonic star is formed. The density becomes peaked at the center, forming a body near1y in hydrostatic equilibrium, surrounded by an accretion shock. The central body contains roughly $10^{-3}$ solar masses and has a radius of about $10^{14} \mathrm{~cm}$. Upon its creation, the protostar may oscillate briefly. Cloud material falls supersonically to the accretion shock, is decelerated, and added to the protostar. The central density continues to rise. When it reaches about $10^{-13}$ $\mathrm{g} / \mathrm{cm}^{3}$, the central temperature also begins to $r$ ise. This phase is illustrated in figures 2 and 3 with the curves from cycle $900\left(t=8.578 \times 10^{12} \mathrm{~s}\right)$. When the central temperature reaches $2000 \mathrm{~K}$, the calculation is terminated. Figure 4 shews the structure at 3500 cycles $\left(t=8.653 \times 10^{12} \mathrm{~s}\right)$, siortly before termination. Real gas physics is needed to go farther because of the importance of $\mathrm{H}_{2}$ dissociation. This has been done in VEGA by making tables of $(\gamma-1) \equiv$ $p / \rho I$ and $c_{v} \equiv I / T$ using the equation of state in Paczynski's stellar envelope program. $10^{\mathrm{V}}$ This pseudo- $\gamma$ and pseudo-specific heat are easy to insert into the code, and they need to be evaluated only at time level $n$ for use in the coefficient matrix. In addition, they are constant over large parts of the $D-T$ plane, so bilinear interpolation is sufficiently accurate.

B. Spherical Blast Wave

The spherical blast wave is a classical test problem for numerical fluid dynamics codes, and it is a much more severe test than piston-driven shocks or shock tubes, In these latter cases, the solutions are piece-wise constant except for the expansion wave in a shock tube, which generally has only modest 


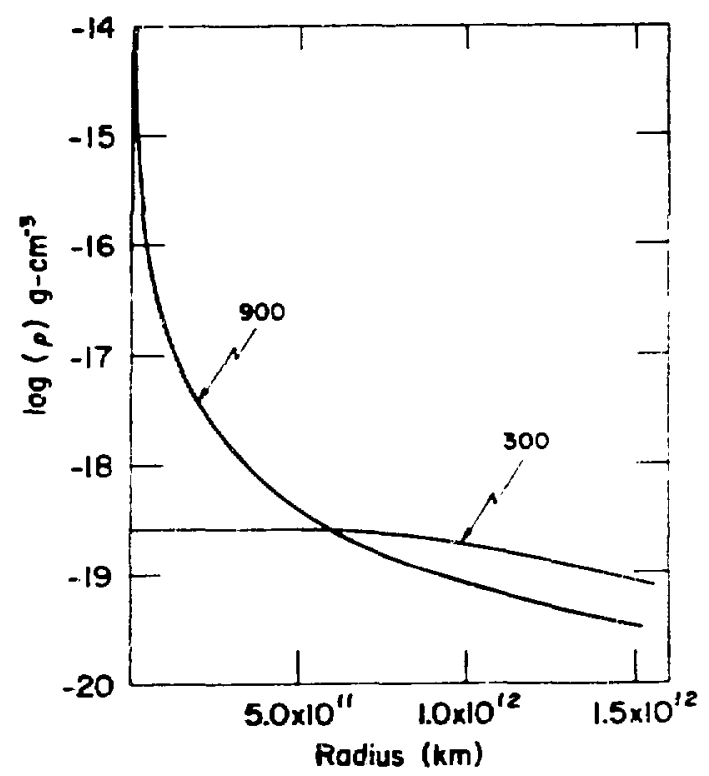

Fig. 2 .

Runs of density at cycles 300 and 900 in the protostar calculation.

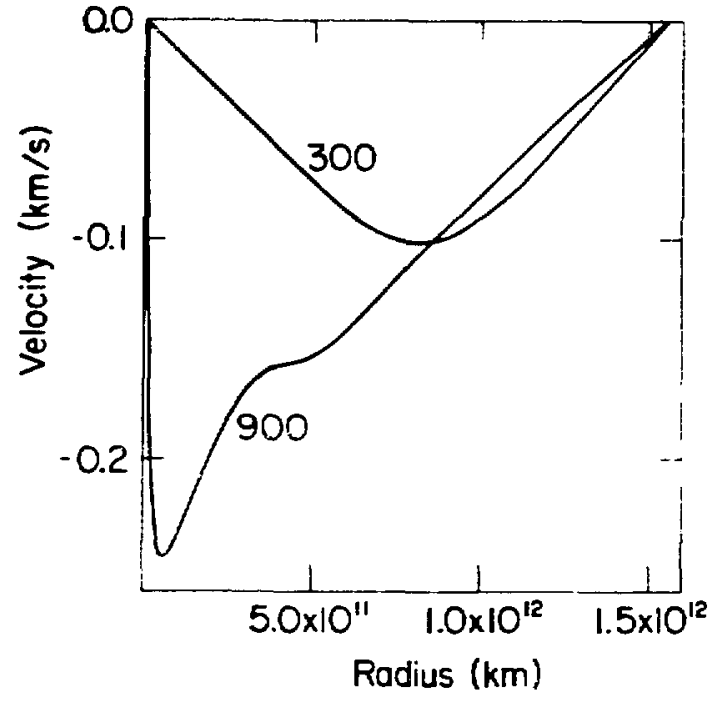

Fig. 3 .

Runs of velocity at 300 and 900 cycles in the protostar calculation.

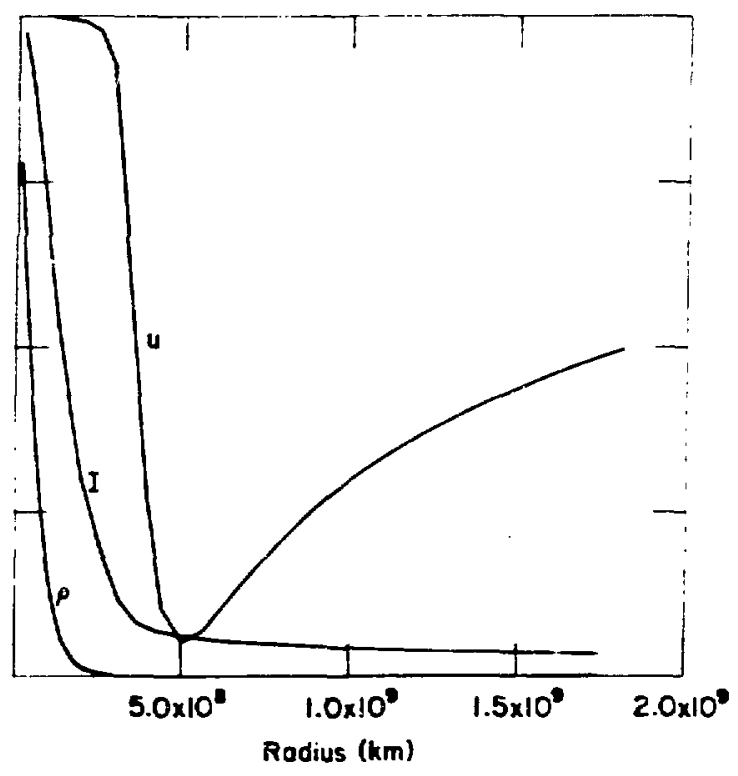

Fig. 4.

The protostar solution at 3500 cycles.

The vertical axis runs from $-3 \times 10^{5}$

to $0 \mathrm{~cm}^{-1}$ for the radial velocity (u), 0 to $1.5 \times 10^{-8} \mathrm{~g}-\mathrm{cm}^{-3}$ for the density $(\rho)$, and 0 to $10^{11}$ for the specif ic internal energy (I). 
curvature. On the other hand, the blast wave solution is sharply peaked, presenting a real challenge for finite difference methods.

The sample problem discussed in this section is based on a $10^{51}$ erg point explosion in a $10,000 \mathrm{~K}$ and $\rho=2.4 \times 10^{-9} \mathrm{~g} / \mathrm{cm}^{3}$ ambient medium with $\gamma=5 / 3$. Appendix $B$ gives UPDATE modifications and the data deck.

Figure 5 shows the numerical solution for the density at two different times. The solid curves are for $\alpha=1.0$ (pure donor cell transport) and the dashed curve is for $\alpha=0.6$. Note that decreasing $\alpha$ reduces the numerical diffusion, thereby sharpening the peaks. Note also the improvement in the densit: jump condition as the wave progresses. This is due to two phenomena. First, the initial condition is not the Taylor-Sedov solution, toward which the solution evolves. Second, and more importantly, the resolution of the sharp selfsimilar peak improves as the radius of the shock grows to include more cells. C. Solar Wind

A simple solar wind model is presented to illustrate use of the code with inflow and outflow boundaries. It also has the left-most vertex away from the

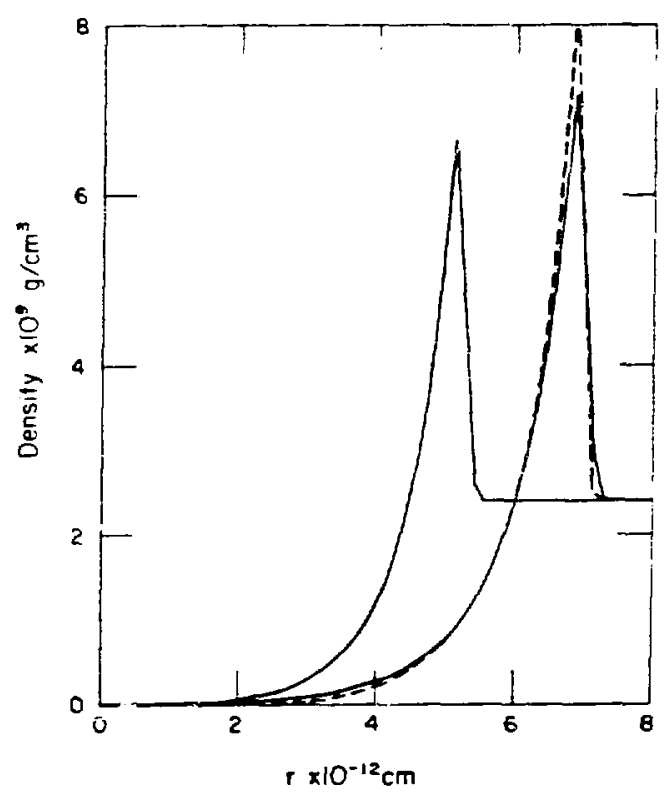

Fig. 5 .

Runs of density for blast wave solutions. The solid curves are for $\alpha=1.0$ at 150 cycles $(t=96.5 \mathrm{~s})$ and 300 cycles $(t=$ $216.5 \mathrm{~s}$ ). The dashed curve is for $\alpha=0.6$ at 300 cycles (t $=212.3 \mathrm{~s}$ ). The peak of the analytical solution is $9.6 \mathrm{x}$ $10^{-9} \mathrm{~g} \mathrm{~cm}^{-3}$ at $\mathrm{r}=7.2 \times 10^{12} \mathrm{~cm}$ at 300 cycles. 
origin. This feature is also useful for running a Cartesian problem merely by making $X(2)$ much larger than the total width of the mesh.

The sample solutions presented here are repetitions of solutions by Hundhausen and Gentry (HG). 9 The first step in this problem is to find a steady state solar wind solution. This could be accomplished by letting the program go through a transient phase. However, the computational effort was minimized by using an inviscid analytical solution as the initial condition. Then transient disturbances were introduced into the solution to represent perturbations by solar flares, and their propagation was followed.

The initial condition is an inviscid adiabatic radial expansion of an ideal gas:

$$
\begin{aligned}
& p=(\gamma-1) \rho I=c_{1} \rho^{\gamma}, \\
& r^{2} \rho u=c_{2},
\end{aligned}
$$

and

$$
u \frac{d u}{d r}+\frac{1}{\rho} \frac{d p}{d r}+\frac{G M}{r^{2}}=0,
$$

where $M$ is the mass of the sun. Substituting (37) into (39) and integrating, we $f$ ind

$$
I+\frac{1}{2} u^{2}+\frac{p}{\rho}-\frac{G M}{r}=C_{3},
$$

which is the Bernoulli equation for this problem. The constants $C_{1}, C_{2}$, and $C_{3}$ are evaluated by specifying the values of all variables at $r=1.25 \times 10^{12} \mathrm{~cm}$, which is outside the critical point of the inviscid solar wind. Elimination of all dependent variables except $\rho$ 'eads to a transcendental equation for $\rho$ :

$$
\rho^{2}\left(c_{3}+\frac{G M}{r}-\frac{\gamma C_{1}}{\gamma-1} \rho^{\gamma-1}\right)=\frac{c_{2}^{2}}{2 x^{4}}
$$


This form is solved iteratively by the program, and then the other variables are found trivially by using equations (37) and (38).

The inflow boundary at the left is straightforward, as can be seen from the UPDATE modifications given in the appendix $C$. However, outflow boun aries are always more troublesome. One simple form that is frequently useful is the continuative boundary, where all gradients are set to zero on the boundary. This procedure is often adequate for supersonic flows, but can reflect unwanted signals into the mesh for subsonic flows. We use an alternate approach, the radiation condition

$$
\frac{\partial c}{\partial t}+u \frac{\partial c}{\partial r}=0
$$

where $c$ is one of the flow variables, in place of the continuative boundary. A small bump in the velocity at the right end of the mesh is strictly a numerical artifact of the outflow boundary. It is slightly smaller using equation (42) than the continuative boundary, and the supersonic outflow prevents it from propagating into the mesh. The user may have to develop a better outflow boundary condition for some problems.

The first numerical solution we ran was the generation of a steady state solution. The analytic solution from equations (37), (38), and (41) was used as the initial condition. The parameters of HG were used. The problem was run beyond the time it takes an element of fluid to cross the mesh, and the numerical and analytic steady states were compared. During the transient, the interface between the fluid originally in the mesh and the fluid that subsequently fluwed into the mesh propagated to the right, showing a small disturbance of increasing amplitude that exited the mesh without reflection. Comparison of the computer-generated plots shows the analytic and computational solutions are almost indistinguishable. Examination of the numerical output shows that the worst errors are near the left boundary where gradients of the variables are the largest. The mesh has been compressed in this region to reduce the error, which has a maximum of $4 \%$ in the pressure. The other variables are computed more accurately, and the accuracy of all variables improves at larger $r$. The second numerical solution was the same as the transient shock problem solved by HG. A disturbance lasting 2.1 hours was introduced into the mesh at 
$t_{0}=2 \times 10^{5} \mathrm{~s}$. This initial period was introduced to allow the inner boundary perturbation to propagate well into the mesh where it could be igrored. This procedure is probably not necessary. Figure (6) corresponds to figures (1)-(3) of HG, and the interested reader is invited to compare the results. The top row of figure 6 shows the solution at $2.0 \mathrm{hrs}$. after $t_{0}$. The analytical solution has a velocity of $1570 \mathrm{~km} / \mathrm{s}$ just behind the shock, in good agreement with our calculation. The velocity jump in HG's figure 1 is a bit too high. Our velocity profile has a spike behind the shock. As we are running with $\alpha=0.5$, this feature is probably a dispersive truncation error. HG show no spike, uggesting that perhaps their solution was obtained using full donor cell transport $(\alpha=1)$. However, Gentry (private communication) has pointed out that at least part of the HG work was done with a scheme that was more closely related to the truncation error cancellation technique of Rivard and collaborators, 11 which is similar in principle to locally computing and applying the minimum $\alpha$ needed in each cell to get numerical stability. This procedure ofter allows signficant dispersive errors to occur, especially near a strong shock, so it is not clear what differencing scheme HG usef to obtain their published results. An unexpected feature of the numerical solutions is the density jump across the shock. The analytic value is a factor of four. Our solution gives a factor of seven, and HG's jump is about the same in spite of the label on their graph showing good agreement with a factor of four jump. The explanation may be that the density gradient is quite large in this region, so shocked material compressed by the correct amount is more than four times as dense as the material ahead of the shock, several cells away.

The second row of figure 6 is taken at $t_{0}+4.3 \mathrm{hr}$. The agreement with HG is better than at $t_{0}+2.1 \mathrm{hr}$, especially in the velocity field. The density jump in the SOLA-STAR solution is still apparently a bit higher than expected.

The bottom row of figure 6 was taken at $t_{0}+20.1 \mathrm{hr}$, as was HG's figure 3. HG's velocity curve is slightly broader and smoother. Their density jump and ours now are close to the desired factor of four, but the velocity and density profiles differ somewhat in detail. Although our solution is in qualitative agreement with that of HG, it is clear that some unanswered questions about these solar wind solutions remain. 

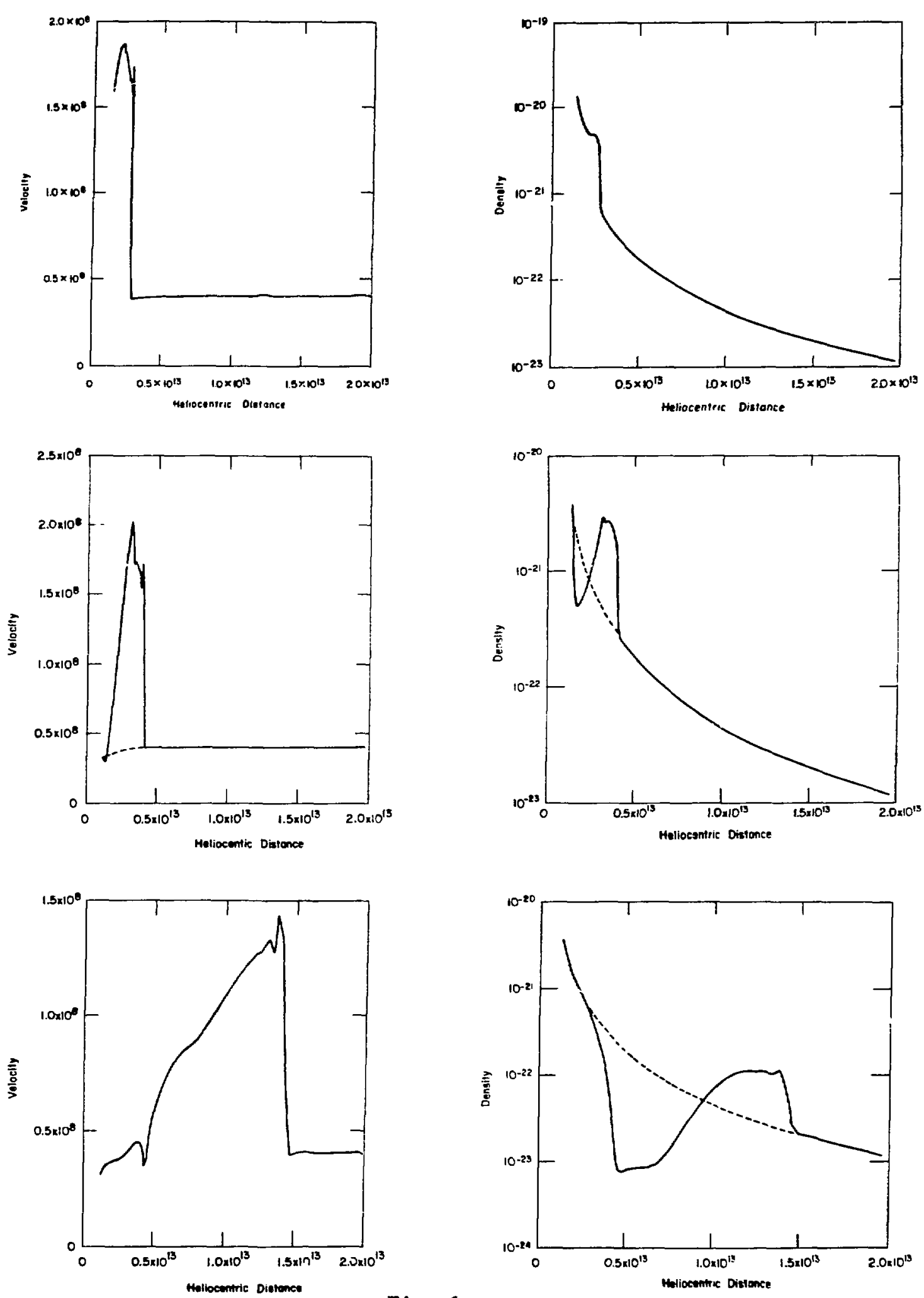

Fig. 6. 

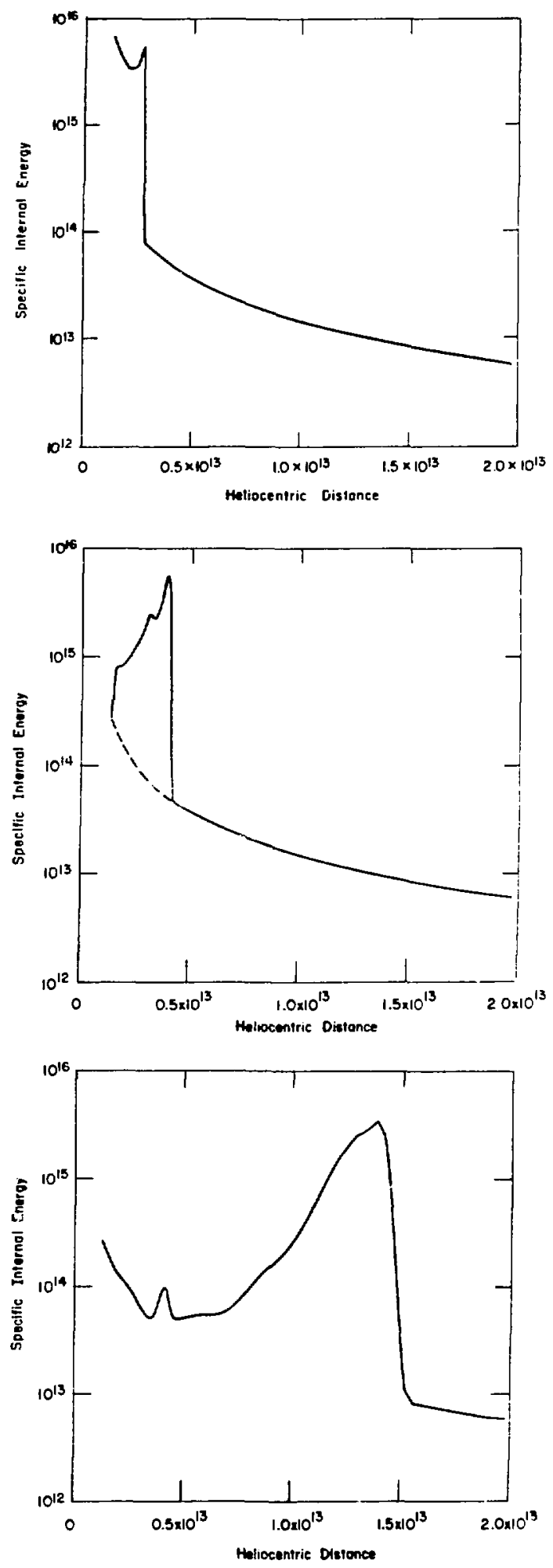

\section{Fig. $6\left(\operatorname{con}^{\prime} t\right)$}

The solar wind solution at $2.0 \mathrm{hr}$., $4.3 \mathrm{hr}$., and $20.1 \mathrm{hr}$. after beginning the shock wave inflow for the top, middle, and bottom rows of figures respectiveiy. Dashed 1 ines indicate the steady state solar wind. 


\section{REFERENCES}

1. Ruppel, H. M. and Cloutman, L. D., "A General Numerical. Fluid Dynamics Algorithm for Astrophysical Applications," Los Alamos Scient if ic Laboratory report LA-6149-MS (1975).

2. Hirt, C. W., Nichols, B. D., and Romero, N. C., "SolA - A Numerical Solution Algorithm for Transient Fluid Flows," Los Alamos Scientific Laboratory report LA-5852 (1975).

3. Clcutman, L. D., Hirt, C. W., and Romero, N. C., "SOLA-ICE: A Numerical Solution Algorithm for Transient Compressible Fluld Flows," Los Alamos Scientific Laboratory report LA-6236 (1976).

4. Hirt, C. W., Amsden, A. A., and Cook, J. L., "An Arbitrary LagrangianEulerian Computing Method for All Flow Speeds," J. Comp. Phys., 14, 227 $(1974)$.

5. Amsden, A. A. and Hirt, C. W., "YAQUI: An Arbitrary Lagranglan-Eulerian Computer Program for Fluid Flow at All Speeds," Los Alamos Scientific Laboratory report LA-5100 (1973).

6. Butler, T. D., Cloutman, I. D., Dukowicz, J. K., and Ramshaw, J. D., "CONCHAS: An Arbitary Lagrangian-gulerian Computer Code for Multicomponent Chemically Reactive Fluid Flow at All Speeds," Los Alamos Scientific Laboratory report LA-8129-MS (1979).

7. Hindmarsh, A. C., "Banded Linear Systems with Pivoting," UCID-30060, Lawrence Livermore Laboratory, Livermore, CA (1970).

8. Larson, R. B., "Numerical Calculations of the Dynamics of a Collapsing Proto-Star," MNRAS, 145, 271 (1969).

9. Hundhausen, A. J. and Gentry, R. A., "Numerical Simulation of Flare-Generated Disturbances in the Solar Wind," J. Geophys. Researsh, 74, 2908 (1969).

10. Paczynski, B., "Envelopes of Red Supergtants," Acta Ascronomica, 19, 1 (1969).

11. Rivard, W. C., Farmer, O. A., Butler, T. D., and O'Rourke, P. J., "A Method for Increased Accuracy in Eulerian Fluid Dynamics Calculations," Los Alamos Scientific Laboratory report LA-5426-MS (1973). 
APPENDIX A

SAMPLE PROBLEM

This appendix provides a listing of SOLA-STAR, a list of main variables, and some information to help the new user convert the code to a non-LASL operating system. We begin by noting that, for the most part, the code is written In ANSI-standard FORTRAN. The CDC computers carry approxiriately 13 digits, which has proved adequate. However for machines with a short word length, such as IBM, it will be necessary to use double precision throughout the code. This is good practice for any hydrodynamics program, but espectally so for SOLA-STAR with its large linear system solver.

SOLA-STAR can be used on quite modest computers. This version of SOLASTAR requires $77 \mathrm{~K}_{8}$ words of memory. However, this number can be reduced if necessary by adjusting the size of dimensioned arrays or by sacrificing the plotting capability. The arrays $\mathrm{B}$ and $\mathrm{AA}$ are $\mathrm{d}$ imensioned for a maximum of $\mathrm{JBAR}=150$ real cells. The grind time, that is the time required to complete one time step for one cell, is $5.3 \mathrm{~ms}$ on a CDC 6400 . This is approximately a factor of 10 longer than for a CDC 7600 and a factor of 2 larger than for a CDC 6600 .

The main systent-dependent feature is the graphics package. Logical unit 7 is the film file, and it can be eliminated if graphics output is not desired. The following is a list of graphical output routines used by SOLA-STAR. These routines rill have to be replaced by the non-LASL user's local equivalent or deleted from the program.

1. CALL $A D V(N F)$ : If $1 \leqslant N F \leqslant 21$, the film is advanced $N F$ frames. Otherwise, the call is ignored.

2. CALL EMPTY: Empty the FILM file buffer onto disk. Unless the run is unexpectedly aborted, this routine is superfluous.

3. CALL LINCNT(N): $N$ is modulo 64 . The next I ine of output directed to the film file is directed to the Nth Iine.

4. CALL SPL $\emptyset T$ (IOP, N, X, Y, ICHAR, IC $\$ N$ ): Standardized plot routine. Four types of grid: I $\emptyset \mathrm{P}=1,2,3$, or 4 glves 1 inear-1inear, linear-log, log-linear, or log-log plots respectively. N successive points are plotted from the tables $X$ and $Y$ (abscissas and ordinates respectively). ICHAR is a code number for a character to be plotted at each point 
$(I C H A R=42$ is a dot). If $I C \emptyset N \neq 0$, the points are connected by straight Iines.

5. CALL WLCH(IX, IY, $\mathrm{CN}, \mathrm{NCH}, \mathrm{NS})$ : Writes large horizontal characters beginning with SC 4020 coordinates (IX, IY). SC 4020 coordinates define a location on the film frame, with $(c, 0)$ at the upper left hand corner and $(1023,1023)$ at the lower right hand corner. NC is the number of. characters to be written beginning with the variable NCH. NS is an integer character size parameter, $1 \leqslant \mathrm{NS} \leqslant 5$.

The sample problems in the Appendixes require mincr changes to the basic code, and it is convenient to specify the changes in SDC UPDATE format, even though many users will not have this software. A statement of the form *INSERT SV.n means insert the FøRTRAN statements between the *INSERT card and the next statement beginning with an * behind statement number SV.n The card *DELETE SV.m,SV.n means delete statements SV.m through SV.n and replace them with any statements between the *DELETE and the next * card. The *IDENT statement merely specifles a name to be associated with the set of modifications and may be ignored by users not using the UPDATE utility.

Table I Iists the major program variables and their definitions. The remainder of this appendix is a program listing and sample output suitatle for testing new SOIA-STAR decks. It is recommended that the new user try these problems to become thoroughly familiar with this code before embarking on his or her own research program. 
AA

AJ

B

CND

DळNM

DळNMפM

DT

DTK

DTMAX

DX

EI

EM

EMC

FMASS

FMøM

FOURPI

G

GAMMA

GRDVL

JBAR

JP1

JP2

JP3

KPR

LFILM

MUISC
Definition

Coefficient matrix (band only) of the linear system.

Areas of cell faces.

Right hand side of the 1 inear system.

Thermal conductivity.

Donor cell parameter $\alpha$ for the $\rho$ and I equations.

Donor cell parameter for the u equation.

Time step.

Maximum allowed value of $|u \delta t / \delta x|$, normally about 0.25 .

Maximum value of DT allowed in the run.

Width of the Innermost two real cells and left hand fictitious ce11.

Specific internal energy I.

Vertex masses.

Cell masses.

Total mass of the system.

Total momentum of the system.

Four times $\pi$.

Gravitational constant.

$Y$, the ratio of specific heats.

Velocity of grid points as fraction of fluid velocity; zero for

Eulerian run, unity for Lagranglan calculation. Can also be

fractional or SUBRøUTINE GRID can be rewritten to provide an arbirtrary, user-chosen grid motion.

Number of real cells.

JBAR +1 .

$\mathrm{JBAR}+2$.

JBAR + 3 .

Get one line summary print every KPR cycles.

Get film output every IFIIM cycles.

Coefficient of viscosity $\mu$. 


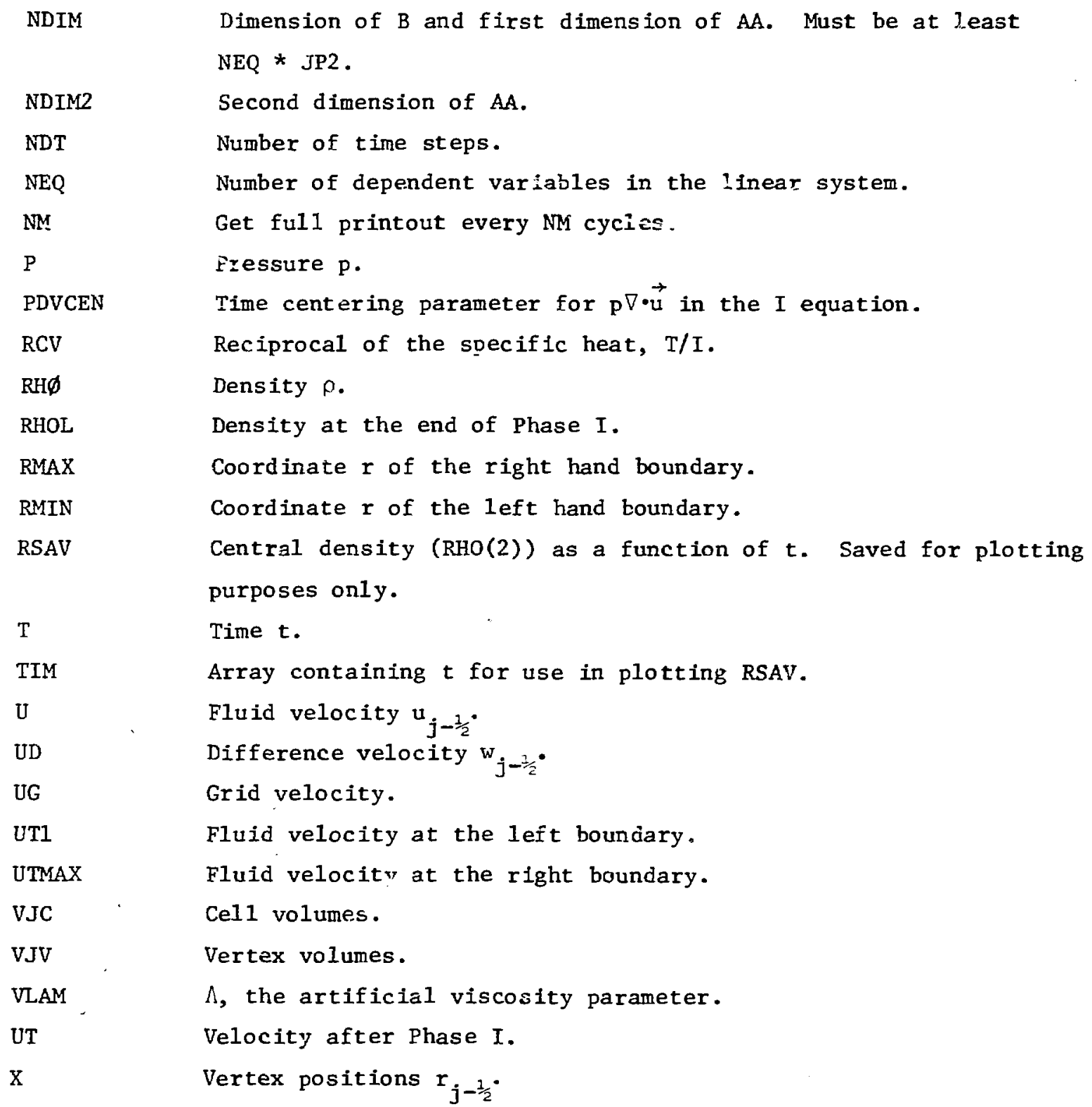


PROGPAY VESA IOUTPUT-100,TAPEj=100,TTY,TAPESA-TTY,

- TAPE5-100I

REAL MUVISC

COMMJN /A1/JBAR,JP1,JP2, JP3,DT,T, N,NDT,FMASS,FMOM,ET,EINT,NMOLFILASV

COMMOV /421 EM(2J)), X(2J) I, U1200), MUVISC(20J)

COMMUN $/ 3 / E I(200), E(200)$, JTMAX,VLAM,DTK

CJYMOV /4) RHJ(200), EYC (20) , P(200),RHJL (200)

:DMMON /AS/ UT(200),JD(200)

CIYMTY $112 /$ GAMMA, JTL,UTMAX, DONM,OJNMOM, GRDVEL

COYMJY /A14/ EMCT(2)J),UG(20J),DPHI(200)

COMMON /ALS/ RCV(20J), CNO(200),R2JR(200), TVJT 4

COMMJN /44O/FJURPI,AJ (2J)), XMR(160)

COMMON /TIMEVI RSAV(2000), TIM(2002), ITIYE

CDYYJY LA,LB,AA $(770,16)$,J SUEJ, VDIM,IP(77O)

OIMENSION ZNU(200), THE $(200)$, ALF $(2) 0)$, BET $(200), 2 K A P(200)$, TAU(200)

JIYENSIJV B(77J), RY(Z)J),RDX(200), AJ: $(200)$

JIMENSIJV RJC(200),VJC (200), RRJC(200),PHI $(200), 2 J(200)$,

$1 Z N(200), Z A(200), R B(200), R E(200), R D(200), V J V(200)$

DATA T.N/O.,OI

DATA FOURPI, G/12.56337062.5.63E-8I

DATA POJCEN/1:I

OATA LFILM,KPR,NM/2E,1,50I

$c *$

$C * * *$ SET UP

$C * * *$

:ALL INPJT

GHI = GAMMA-1.

TNOT 4 = (EI (JPI) *RCV $(J P 1)) * 4$

WRITE $(3.230)$ TN.JT

WRITE $(7,230)$ TNOTA

i *** LA IS NJYBER DF ELEMENTS TO LEFT JF DIAGONAL, LG TO RIGHT

$L A=5$

$L B=5$

VEN $=5$

NO $(Y=77)$

VDIML-IS

$J B N E Q \cdot J P L$ *NEJ

$D D 10 \mathrm{~J}=2, \mathrm{JP} 2$

$5 D x(7)=1 \cdot 1(x(7+1)-x(7))$

$\sin (7)=.5 *(x(7)+i(j+1))$ 
$\operatorname{AJC}(J) \cdot 3 . * \operatorname{RJC}(J) * R J C(J)$

$\operatorname{RRJC}(J)=0.5 / R J C(J)$

$5 A(7)=5 J x(7)+5.4557:(7)$

$R B(J)=R J X(J)-2 \cdot$ \&RJC $(J)$

$\angle C(J)=(5 D \times(J)+R R J C(J)) * R R J=(J)$

$B D(J)=(\operatorname{RDX}(J)-R R J C(J)) * \operatorname{R} J C(J)$

$R J=(7) * x(7)+1) * 3-x(J) * 3$

$\operatorname{EMC}(2)=5+0(J) * V]=(7)$

10 SONTINUE

₹JC(1)=0.5*(x(1)+X(2))

$=* * * 22 J R(2)=0$ TO YAKE DIIJR=0 AT JRIGIV

र2OR $(2) \cdot 0$.

$3020 \mathrm{~J}=3 . \mathrm{JP} 2$

$\operatorname{RODR}(7)=1 \cdot 1(x(7+1)-x(7-1)$

$A J(J)=3 \cdot * x(J) * x(J)$

VJV $(J)=2 j=(J) * 3-2 J C(J-1) * 3$

$20 E M(J)=0.5 *(E M C(J-1)+E M C(J))$

$A d(2)=3 . * x(2) * x(2)$

$A J(J P 3)=3 . * x(J P 3) * x(J P 3)$

$V J V(2) * 2 J=(2) * 3-x(2) * 3$

$E M(2)=0.5 * E Y C(2)$

CALL OJVDJCT

EALL OJTPUT

DTt $=0 t$

IT IME $\triangle 0$

$\sum * * *$

C $* *$

HYORJUYVAMIES LJJP

DO $200 \quad V=1, N D T$

ITIME=ITIME+I

IF (ITIYE.GT.5OJ) ITIYE:I

$D T=A M I N I(D T T, I, I+J T)$

$T=T+D T$

TEYPEAEI ( 2$) \leftrightarrow R C V(2)$

IF(YJO(VOKPR).EQ.J) WRITE (6.220) DT,T.N.R4J(2).U(15),TEMPE

IF (MJD(N, KPR),E2.O)ARITE $(T, 220)$ DT,T,N,RHO(2),U(15),T EMPE

IF (TEMPE.GT. 3000.) CALL EXIT

$J B M=J A A R-1$

$3 E T(2)=0$.

BET (JPZ) $X(J P 2) * 2 * 20 \times(J P D)$ 
$D P H I(2)=0$.

$S U Y=0$.

DUG $A F J U R D I * G / 3$.

DO $30 \mathrm{~J}-3, \mathrm{JPI}$

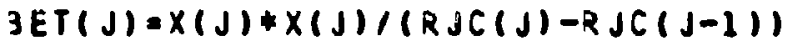

SUY $=S U Y+E Y C(J-1) * D U G$

JPHI $(J)=S J M /(X(J) * x(J))$

30 COVTIVIJE

DPHI (JPZ) $=(S U M+E M C(J P I) * D U G) /(X(J P Z) \neq X(J P Z))$

SMAX $=A B S(J P H I(J P Z))$

CAL! CJNDUCT

₹2JR (JP3) $=0$.

DJ $30 \mathrm{~J}=2, \mathrm{JPL}$

$T I=U(J+1)-U(J)$

$15=U(J+1)+U(j)$

r $3=0$.

IF (J.NE,JPZ) T3-OT/EMC(J)

$20=T 1 * R) \times(J)+2 . * T 2 * 2 R J C(J)$

$T 4 *-0 T /(1 \cdot+0 T * 20)$

$T S=2 * 41 * R 0 \times(J) * 2$

$T 6-4, * T 2 * R 2 J(J) * 2$

$3 E T(J)=(T I * R D X(J)) * * 2+2 * *(t 2 * R R J(J)) * 2$

LNU $(J)=0.5 * T 3$

$A L F(J)=2 \cdot * 13 * 4 J \operatorname{SISC}(J) * V J C(J)$

THE $(J)-J(J+1) * A J(J+b)-U(J) * A J(J)$

$\operatorname{ZKAP}(J)=T 5+T 6$

$T A U(J)=T 5=T 6$

SM(J)-JT/ER(J)

PHI (J)-T4*R+O(J)

30 SONTINUE

C ** YATRIX STRUCTJRE

$C * * *$

C *** FORMAT BLOCK

C ***

C RHJ

U

P

I

$K, L+2$

$\begin{array}{lr}\text { SV } & 82 \\ \text { SV } & 83 \\ \text { SV } & 84 \\ \text { SV } & 85 \\ \text { SV } & 86 \\ \text { SV } & 87 \\ \text { SV } & 88 \\ \text { SV } & 87 \\ \text { SV } & 90 \\ \text { SV } & 91 \\ \text { SV } & 92 \\ \text { SV } & 93 \\ \text { SV } & 94 \\ \text { SV } & 95 \\ \text { SV } & 96 \\ \text { SV } & 97 \\ \text { SV } & 99 \\ \text { SV } & 99 \\ \text { SV } & 100 \\ \text { SV } & 101 \\ \text { SV } & 102 \\ \text { SV } & 103 \\ \text { SV } & 104 \\ \text { SV } & 105 \\ \text { SV } & 106 \\ \text { SV } & 107 \\ \text { SV } & 108 \\ \text { SV } & 109 \\ \text { SV } & 110 \\ \text { SV } & 111 \\ \text { SV } & 112 \\ \text { SV } & 113 \\ \text { SV } & 116 \\ \text { SV } & 115 \\ \text { SV } & 116 \\ \text { SV } & 111 \\ \text { SV } & 118 \\ \text { SV } & 119 \\ \text { SV } & 120 \\ \text { SV } & 121 \\ & \\ & \end{array}$


0] $70 \quad[-1, N D I M$

DO $70 \mathrm{~J}=1, \mathrm{NOI} 42$

$90 \quad A A(I, J)=0$.

$A A(2,6)=1$.

$\triangle A(4,7)=1$.

$K=1-N E Q$

$L=L A+1$

OIVR=J.

$\triangle R T P P=0$.

$D O 12 J j=2, J P 2$

$K=K+N E Q$

$\triangle A(<, L)=-G \times 1+E[(J)$

$A A(K, L+2)=1$.

$A A(K, L+3)=-G M I * R H J(J)$

JIVL=JIV

DIVR-J.

IF (J. $L T_{E} J P\{)$ OIVR=(AJ $\left.(J+1) * U(J+1)-A J(J) * U(J)\right) / V J E(J)$

IF IDIVR . GT O O.I DIVR=0.

ARTPMATRTPP

ARTPP $\vee L L M \# R H J(J) * D I V R * D I V R /(R D X(J) * R D \times(J))$

IF(J .E2. 2) GO TJ 100

1)

$A A(K+1, L-5)=-4$ * *VJV $(J) * R M(J) * M U V I S C(J-1) *(R 2 O R(J) * R O X(J-1)-R C(J-1) S$

$A A(K+L, L-4)=-2 M(J) * A J(J)$

$A A(X+1, L)=10+4, * V J V(J) * R Y(J) *(M U V[S C(J) *(R \geq O R(J) * R O X(J)+R E(J))$

1 +MUVISE $(J-1) *(R 2 O R(J) * R D \times(J-2)-R D(J-1) 1)$

$A A(<+1, L+1)=R M(J) * A J(J)$

$A A(K+1, L+5)=-40 * V J V(J) * R M(J) * 4 U V I S C(J) *(R 2 D R(J) * R D X(J)+R O(J))$

$A A(K+3, L-5)=2 \cdot * C V O(J) * R C V(J) * R 2 O R(J)$

$A A(K+3, L+1)=1$.

$A A(K+3,, L)=-2, * C N O(J) * R C V(J+7) * 25 D R(J)$

100 AA $(<+4, L-3)=A L F(J) * T A U(J)-P O V C E N * Z N U(J) * P(J) * A J(J) * 2$.

$A A(K+4, L-2)=2, * Z N U(J) * T H E(J)$

- *POVCEY 


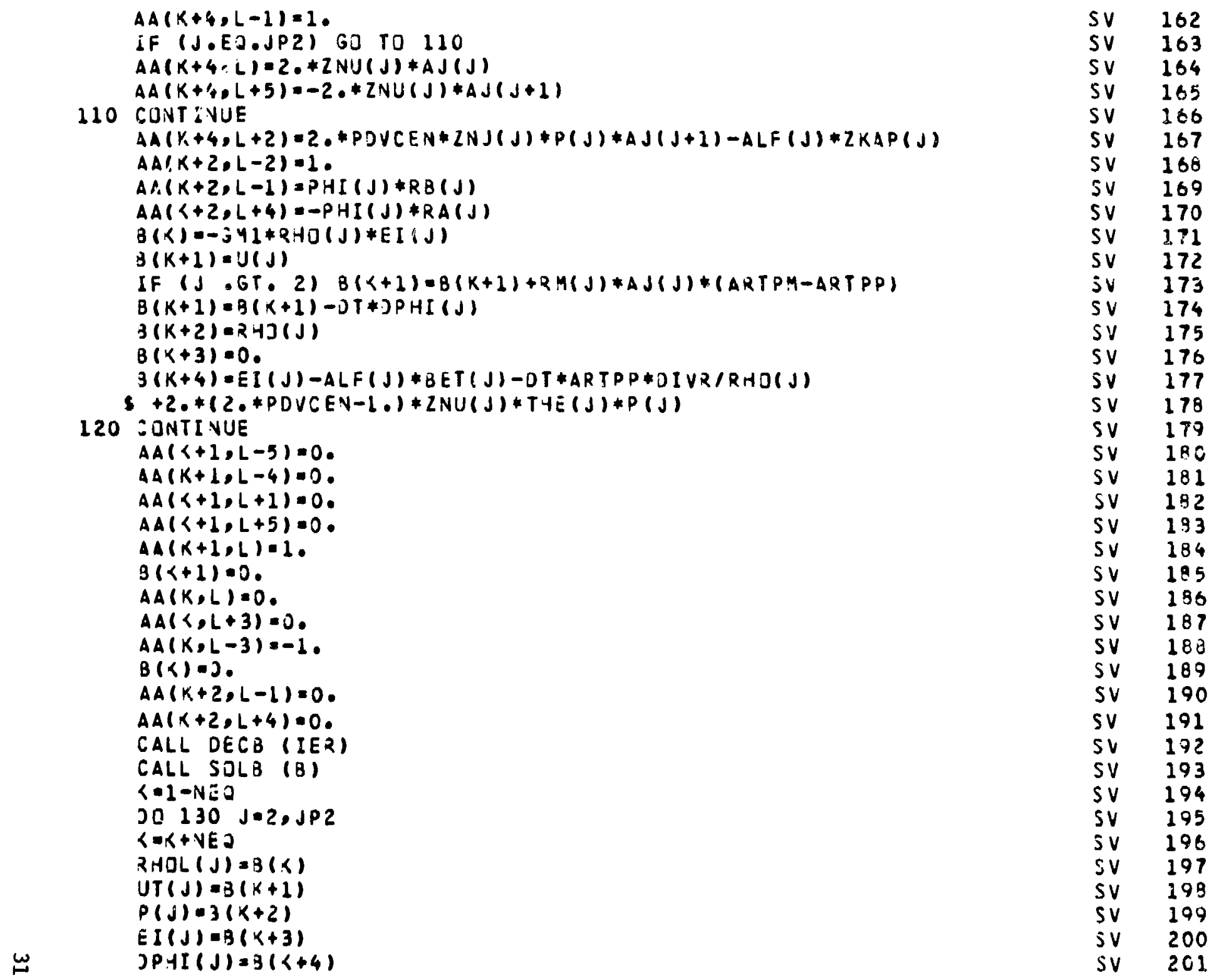


RHJL $(1): R H O L(2)$

ZHOL $(J P ?)=R+J L(J P L)$

$c * * *$

$C * *$

zezone section

C $\neq *$

SALL BRID

$00150 \mathrm{~J}=2, \mathrm{JPI}$

OUBI-ABS $(D O N M)$ \&I I GN $(1, \ldots U D(J))$

DUBZ=-A3S(DONM) *SIGN $(1, \rho U D(J+1))$

$E M E T(J)=E M C(J)-0.5 * D T *(U)(J) * A J(J) *((1 .+D U B 1) * R+4 O(J-1)+(1 .-D U B 1) * 5 V$

$[R H] L(J))-J D(J+1) * 4 J(J+1) *((2,+D \cup 32) * Z+J L(J)+(1 \cdot-D U B 2) * R+O L(J+1))) S V$

$E(J)=(E I(J) * E Y C(J)-.5 * J T *(J O(J) * A J(J) *((1,+D U B I) * R H O L(J-1) * E I(J-1) S V$

$1+(\lambda \bullet-D J B 1) * R H O L(J) * E I(J))-U D(J+1) * A J(J+1) *((1 \bullet+D J B 2) * R H O L(J) * E[(J) S V$

$2+(2 \cdot-J J 35) * 2 H J L(J+1) * E I(J+1))) J / E$ YCT (J)

150 COVTIVJE

EMCT $(J P Z)=E M C(J P ?)$

$E(J P Z)=E I(J P 2)$

$30160 \mathrm{~J}=3, \mathrm{JP} 2$

JUB $1=-A B S(D O N M J M) * S I S N(1, . U D(J)+U D(J-1))$

$D J 32=-A B S(D O N M O M) * S I G N(1$ OUD $(J)+U O(J+1))$

$U(J)=E M(J) * J T(J)-.25 * D T *(R+O L(J-1) *(J D(J)+U D(J-1)) * A J C(J-1) *(1)+$ $1 D \cup B 1) * U T(J-1)+(1 .-0 \cup 31) * J T(J))-R+D L(J) *(U D(J)+U D(J+1)) * A J C(J) * 1$

$5(1 \cdot+3 \cup 35) \div U T(3)+(1 \cdot-0) B 2) *(1)(J+1)))$

150 CONTINUE

$U(J P L)=j$.

$D P_{A}+I(2)=0$.

$20170 \mathrm{~J}=2, \mathrm{JP2}$

$50 \times(j)=1.1(x(j+1)-x(j))$

$3 J C(J)=.5 *(x(J)+x(J+1))$

$A J C(J)=3 . * R J C(J) * R J C(J)$

$R R J=(J)=0.5 / R J C(J)$

$R A(J)=R D \times(J)+2 . * R R J=(J)$

$5 B(J)=R J \times(J)-5, * R R J C(J)$

$R C(J)=(5) \times(J)+\operatorname{siJ} J(J)) * R R J C(J)$

$\{J(J)=(5) \times(J)-R R J C(J)) * R \cdot J=(J)$

$\operatorname{VJC}(J)=x(J+1) * 3-x(J) * 3$

EME $(J)=E$ YCT $(J)$

SHU(J)=EMC(J)/VJC(J) 
$E I(J)=E(7)$

173 CQVTINUE

$R J C(1)=0.5 *(x(1)+x(2))$

DTT $=$ DTMAX

DO $190 \mathrm{~J}=3, \mathrm{JP2}$

$\operatorname{RiDR}(7)=1.1(x(7+1)-x(7-1))$

$A J(J)=3 . * x(J) * x(J)$

$\operatorname{JU}(J)=R J C(J) * 3-R J E(J-1) * 3$

$E M(J)=0.5 *(E M C(J-1)+E M C(J))$

$U(J)=U(J) / E Y(J)$

IF (U(J),NE.O.) DTT=AMINI(OTT,OTK*(X:J+I)-X(J))/ABS(U(J))

100 CONTINUE

$V J V(2)=2 J C(2) * * 3-x(2) * * 3$

$\operatorname{AJ}(2)=3 . * x(2) * x(2)$

$A J(J P 3)=3 . * X(J P 3) * X(J P 3)$

$E M(2)=0.5 * E M C T(2)$

$U(2)=U T 1$

RHQ (1) $=2 H O(2)$

$R H J(J P 2)=R H O(J P 1)$

$E I(1)=E I(2)$

$6+*$

C *** TIME ACCOUNTING

C $* * *$

IF (MOD (N,NM) .NE.O .AND. MJD(N,LFILM).NE.O .AND. V.GT.I)

S GJ TO 172

ETOO.

EINT $=0$.

FMASS $=0$.

$F M O M=J$.

DJ $19 J \mathrm{~J}=5, J P Z$

$F M O M=\bar{F} M J M+E Y(J) * U T(J)$

$E T=E T+0.5 * E H(J) * U T(J) * U T(J)$

IF (J.EO. JPZ) GO TO 170

$E T=E T+E Y C(J) * E I(N)$

EINT -EINT+EMC (J)*EI (J)

FMASS $=F Y A S S+E Y E(J)$

$F M O M=F M O M+E M(J)+U T(J)$

190 CONTINUE

$\boldsymbol{\omega}$

ET $\triangle$ ET \& TOUZPI / 3 .

EINTAEIVT *FOURP I / 3 .

265

266

267

268

269

270

271

272

273

274

275

276

277

279

279

280

281 


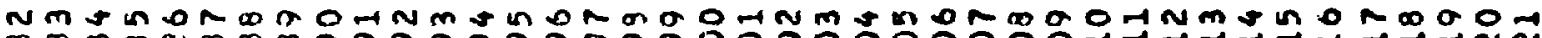

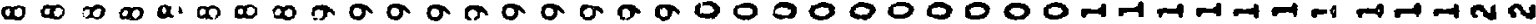

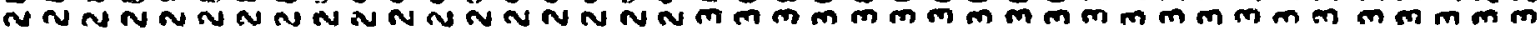

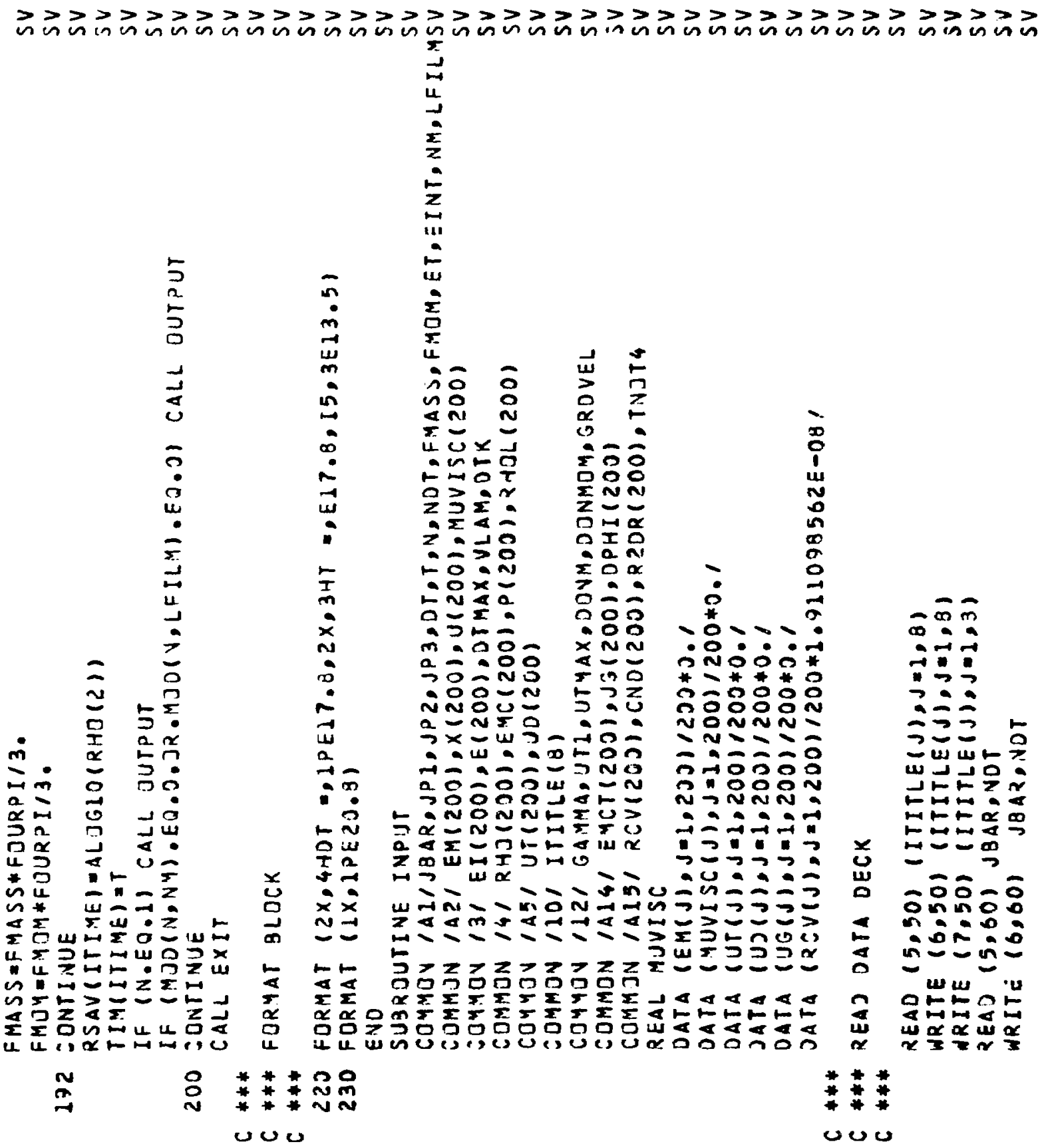


Nm

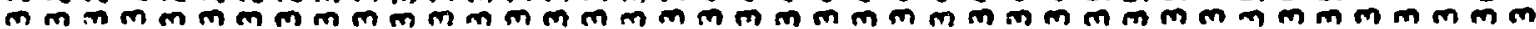

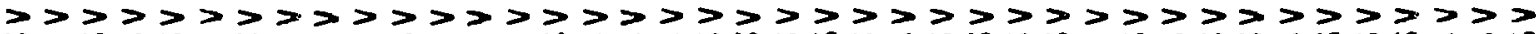
n

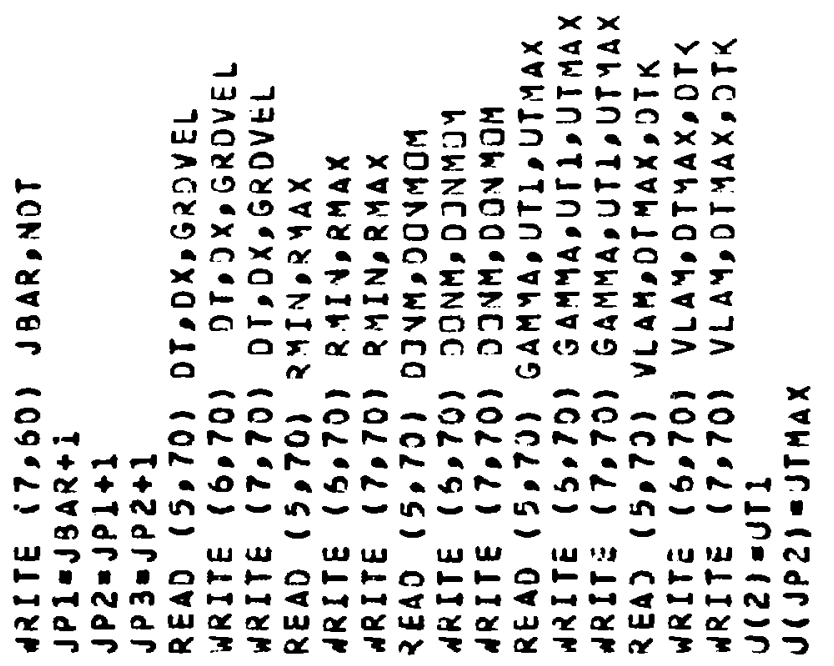

$\leftarrow$

m

w

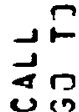

in

메 $x y$

$\rightarrow \quad 20$

a $\quad$ \&

$\pm \quad \because$

$\rightarrow \vdash 0 \square$

- $\mapsto b$

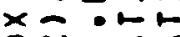
$a n=a$ $a x a x$ $\rightarrow \rightarrow \square$ $n-5 \mapsto r$ a $\times$ a I $\rightarrow-\infty I$ $=0-\alpha x$ a $40-$ $0 \cdot 2 x x$ $\rightarrow$ in $x<x$ - 0.12

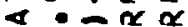
r.NO - apin $m \geq 0$ a 4 $\rightarrow-2$ $00 \geq 1$ $I$
un
$=$
5
$=$ 7 in 1 $\infty \div>2$

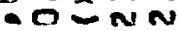

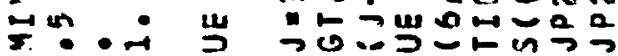

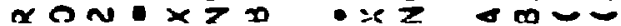

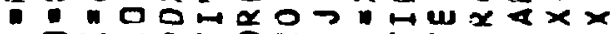

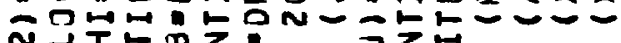

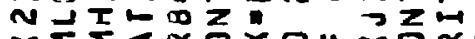

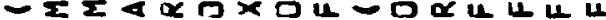

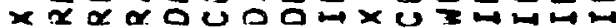

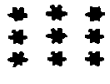

0

a 
30 COVTIVUE

$x(1) \cdot 2 * x(2)-x(3)$

$=* * *$

C *** INITIALIZE DEPENDENT VARIABLES

C ****

DJ $40 \mathrm{~J}=1, \mathrm{JP2}$

$R H O(J)=1.10 E-17$

$R H J(J)=1.26 E-17$

$P(J)=3.676 E-11$

$P(J)=4.39758 E-11$

$U(J)=0$.

$E I(J)=P(J) /((G A M A A-1) * R A D.(J))$

$4 C$ CDVTIVUE

$U(2)=U T 1$

$U(7 P 3)=J(J P L)$

$U D(J P 3)=J$.

$U T(J P 3)=0$.

CALL SETKAP

$c$ RETURN

SO FORMAT (BALO)

OU FORMAT (IOH

II 0.104

TO FORMAT IIJH

,E10.3.10H

. I101

1 E 10.31

BO FORMAT (IX, *RATIO, RMAX FROM MESH GEN\$, IP3ElE. B)

END

SUBROUTIVE SETKAP

COMMON/KAPASKAP $(51,31)$, XMF, YMF, ZMF

REAL SAP

$c$

C *** READS GOB DPACITY JECK

READ $(5,100)$ XMF,YMF

100 FORMAT (10F3.5)

ZMF $=1,-X M F-, M F$

$I=0$

$x z=0$

$30 J$ COVTINUE 
$I=I+1$

IF $(I . G T, 51)$ GJ TJ 304

301 FORMAT $(1 X, I 5,14 F 5,2)$

$K 2=\times 2+1$

$\operatorname{READ}(5,3 J 1) K 1,(\langle A P(I, J), J=1,24)$

IF $(K 1 \cdot N E \cdot K 2)$ GJ TJ 322

$K 2=K 2+1$

READ $(5,301) K 1,(K A P(I, J), J=15,23)$

IF (KL N NE. (2) GO TO 302

$<2=<2+1$

READ $(5,301) K 1,(K A P(I, J), J=29,31)$

IF (KL -NE. K2) GJ TJ 302

SO TO 300

302 CONTINUE

303 FORMAT (1X,21 HARONG JPACITY CARD,K=I3)

WRITE $(6,303) \mathrm{K2}$

CALL EXIT

304 : ONTINUE

RETURY

END

SUBRDUTIVE UUTPJT

REAL MUVISC

COYMJV /AI/J3AR, JPI, JP?, JP3, OT, T, N,NDT,FMASS, FMJM, ET, EINT, NM, LFILMSV

COMMON $/ 421$ EM(200), X(200),U(200), MUVISC(200)

COMMON /3) EI(2O0),E(200),DTMAX, VLAM,OTK

SOMMON 141 RHO(200), EME $(200), P(200), R$ HOL $(200)$

COHMJY /AJI UT(200), UD(200)

COMMJN $/ 10 /$ ITITLE(S)

COMYOV $/ 12 /$ GAMMA, JTL, UTYAX, JTVM, DONMOM, GRDVEL

SOMMON /AI4/ EMCT(200),JG(?00),DPHI(200)

COYMJN 1415/ RCV(200),CND(200),R2DR(20)),TNOT4

CQYMON /A4O/F]URPI, AJ $(200)$, XMR(160)

SOMMON /TIMEVI RSAV(2060), TIM(2000), ITIME

DIMENSION AL $(200), B L(200), C L(200)$

JIMENSL IN XC(2)J), TEM(200)

DATA UTITLE/IOHX-VELJCITYI

JATA $(J P H I(J), J * 1,200) / 200 * 0.1$

DATA PTITLE/BHPRESSURE/

DATA $(X, Y R(J), J=1,160) / 160 * 0.1$ 
$0025 \mathrm{~J}=2, \mathrm{JP2}$

IF $(J \cdot G T \cdot 2) \times M R(J)=X M R(J-1)+F$ JURPI*F,YC(J-1)/3.

DPHI $(J)=-F$ OURPI *AJ $(J) * D P H I(J) / 3$.

$T E M(J)=E[(J) * R C V(J)$

25 CDVTIVJE

IUMI $N=6$

IUYAX $=7$

IF (N.LE.I) GO TO IJ

IF (MOD(N,NM) .NE.O) IUMI $Y=$ ?

IF $(M J D(N, L F[L M), N E . O)$ IUMAX $=6$

IF (IUMIN口GT.IUMAX) IUMAX=7

102020 IU IUMIN.IUMAX

WRITE $(I U, 40)$ EIVT,ET, FMASS, FMOM, N

IF (IU.EQ.T) CALL ADV (1)

WRITE $(I U, 50)$

ARITE (IU,6O)(I,X(I),U(I),RHO(I),EI(I),P(I), SNO(I),OPHI (I),

1 XMR(I),TEM(I),I=2,JP2)

$* * *$

o cONTINUE

C *** GRAPHIZAL LIUTPUT

$C * * *$

IF (IJMAX -ED. S) RETURN

CALL SPLOT $(1, J P 1, X(2), U(2), 42,1)$

SALL NLEH $(0,0,56, I T I T L E, 2)$

CALL NLCH $(0,25,10$, UTITLE,1)

CALL LINCNT 1601

NRITE $(7,70) T, N$

DJ $30 \mathrm{~J}=2, \mathrm{JPI}$

$x \in(7)=.5 *(x(7)+x(7+1))$

$A L(J)=A L D S 10(R H J(J))$

$B L(J)=A L J G 2 O(E[(J))$

$C L(J)=A L D G I O(P(J))$

30 SONTINUE

CALL SPLOT (1,JPI-1,XC(2),P (2),42,1)

CALL WLEH $(0,0,56$, ITITLE, Z)

CALL NLCH $(0,25,8$, PTITLE,1)

CALL LINENT (SO)

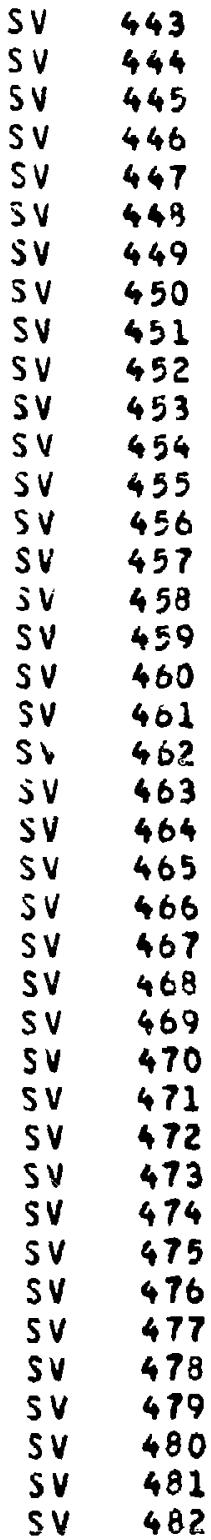


ARITE $(7,70) T, V$

CALL SPLOT $(1, J P 1-1, X C(2), E I(2), 42,1)$

CALL NLE+ $(0,0,55$, ITITLE, 2)

CALL NLST $(0,2 j, 3, X I T L E, 1)$

CALL LINENT $(60)$

NRITE $(7,70) T, V$

CALL SPLJT (1,JP1-1,XC(2),RHJ(2),42,1)

CALL WLCH $(0,0,56$, ITITLE, 2$)$

CALL ALCH $(0,25,7, R+0 T I T L, 1)$

CALL LINCNT $(03)$

WRITE $(7,70) T, N$

CALL SPLOT $(1, J P 1-1, X C(2), C N D(2), 42,1)$

CALL NLEH $(0,0,53$, ITITLE, Z)

CALL NLOH $(0,25,3,3$ HCND,1)

CALL LINCNT (SO)

WRITE $(7,70) T, N$

IF (N.GT.10) CALL SPLOT (2,ITIME,TIM,RSAV,42,1)

IF (N.3T.10) CALL WLCH $(0,25,3,3$ HRHO VS T,1)

CALL LINCNT (60)

WRITE $(7,70) T, N$

$J B A R=J P 1-1$

GALL SPLJT $(2, J B A R, X C(2), A L(2), 42,1)$

CALL WLCH $(0,25,7, R H J T I T L, 1)$

CALL SPLOT $(2, J B A R, X C(2), 3 L(2), 42,1)$

CALL $H L O+(0,25,3, X I T L E, I)$

CALL SPLOT $(2, J B A R, X C(2), C L(2), 42,1)$

CALL NLC+(0,25,8,PTITLE, I)

$X J C L=1, \tilde{E}-3 * X(J P \geq)$

OO $7000 \mathrm{~J}=2, \mathrm{JP} I$

$J C L=J$

IF $(X(J), G T \cdot X J C L)$ GJ TJ 7001

7000 CONTINUE

7001 COVTIVJE

IF (JEL EEO. JOI .DR. JCL .LT. 3) GDTD 7002

CALL SPLOT(1, JLL, X $(2), U(2), 42,1)$

CALL NLCH $(0,25,10$, UTITLE,1)

CALL SPLUT(1,JCL-1,XC(2), $P(2), 42,1)$

CALL NLOH(0,25,8,PTITLE,1)

CALL SPLIT (1,JEL-1,XC $(2), E I(2), 42,1)$

CALL ALCH(0,25,3, XITLE,1)

$\begin{array}{ll}\text { SV } & 483 \\ \text { SV } & 484 \\ \text { SV } & 485 \\ \text { SV } & 485 \\ \text { SV } & 487 \\ \text { SV } & 488 \\ \text { SV } & 489 \\ \text { SV } & 490 \\ \text { SV } & 491 \\ \text { SV } & 492 \\ \text { SV } & 493 \\ \text { SV } & 494 \\ \text { SV } & 495 \\ \text { SV } & 476 \\ \text { SV } & 497 \\ \text { SV } & 498 \\ \text { SV } & 499 \\ \text { SV } & 500 \\ \text { SV } & 501 \\ \text { SV } & 502 \\ \text { SV } & 503 \\ \text { SV } & 504 \\ \text { SV } & 505 \\ \text { SV } & 536 \\ \text { SV } & 507 \\ \text { SV } & 508 \\ \text { SV } & 509 \\ \text { SV } & 510 \\ \text { SV } & 511 \\ \text { SV } & 512 \\ \text { SV } & 513 \\ \text { SV } & 514 \\ \text { SV } & 515 \\ \text { SV } & 516 \\ \text { SV } & 517 \\ \text { SV } & 518 \\ \text { SV } & 519 \\ \text { SV } & 520 \\ \text { SV } & 521 \\ \text { SV } & 522 \\ & \\ & \end{array}$


CALL SPLOT(1, JCL-1,XE(2),RHO(2),42,1)

CALL ILCH $10,25,7,2, H T T T L, 1)$

7002 CONTINUE

WRITE $(57,70)$ T,N

IF ITEM(2) .GT. 2000.) CALL EXIT

RETURV

$\check{c}$

C*** FORMAT BLDCK

C $* * *$

40 FORHAT $11 x, 17$ HINTERNAL ENERGY $: 1$ PE 13.6,

1 15H TJTAL ENERGY =,E13.6.7H MASS $\#$ E13.6.114 MOMENTUM =.513.6,

18 H CYCLE $\because$ I 71

50 FORMAT $(4 x, 14 J, 7 x, 1 H X, 12 x, 1 H U, 11 X, 3 H R 40,10 X, 2 H E I, 11 X, 14 P$,

- 10X,3HCND,11X,1HL,10X,2+MR, 11X,1HT)

6O FORMAT $(1 X, 13,1 P 9 E 23,5)$

70 FDRMAT (SH TIMEO,IPE10,3,7 CYELEE,I5)

END

SUSZJUTIVE GRID

REAL YUVISC

SOMMON /AI/JGAR,JPI, JPR, JPB, JT,T, N, NDT,FMASS,FMOM,ET, EINT, NM, LFILYSV

COMMOV /AZI EM(20)),X(200), U(200), MJVISE(20J)

COMMON /A5/ UT(200),JD(200)

COMMOV 1121 GAYYA,UTLOUTMAX,DOVM,DONMOM,GRDVEL

DOMMON /A14/ EYC: (200), JG(?00),DPHI(200)

C. $* * *$

C ** JEFINE GRID VELJCITY UG, REZJNe (DIFFEzENCE) VELUCITY LD,

C** AND NEN VERTEX POSITIONS X.

C ** GROVEL = 0., EJLEZIAN. GRDVEl I. LAGRANGian.

C $* * *$

$J 010 \mathrm{~J}=\mathrm{z}, J P z$

$J G(J)=G R D V E L * J t(J)$

$\operatorname{UD}(J)=U j(J)-U T(J)$

$x(7)=x(7)+D T * U \in(j)$

10 CONTINUE

$X(J P 3)=5, * x(J P S)-x(J P I)$

$x(1)=2 * * x(2)-x(3)$

RETUR V

END

REAL FUNCTION KAPPA(ZJI,TE)

C *** T LIMITS JF TABLE ARE LOG(T)=3.3 AND 3.2 
C *** RHJ TABLE LIMITS ARE 1.E-12 AND 1000. COMMOV/ $\angle A P A / K A P(51,31), X M F, Y M F, Z M F$

REAL KAO, $\angle A P P$

INTEGER DI,TI

OATA JUSTKIO.15I

C *** DUST JPACITY ONLY

KAPPA $=$ DUSTK

IF (TE. LE. 1500.) RETUR.V

C ** GAS DPAEITY

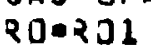

$0=2.0 * A L O B 10(k J)+25.0$

$J I=I N T(D)$

$D=D-D I$

$T=20 . * A L D G L O(T E)-65$.

IF $(T \cdot 3 T \cdot 35) T-.35 .+(T-35) *$.

$T I=I N T(t)$

$t=t-t i$

IF IDI .SE. 1) GJ TO 30

$\mathrm{OI} \cdot \mathrm{I}$

$j=0$.

30 CONTIVJE

IF IDI.LE. 30 ) GO TO 31

$D I \oplus 30$

$0 \cdot 1$.

31 CONTIVUE

IF (TI .GE. Li GO TO 32

$T i=1$

$r=0$.

32 CJVTIVUE

IF ITI.LE. 50130 TJ 33

$T I=50$

$r \cdot 1$.

$33<A P P=(1,-T) *((1,-D) * K A P(T I, O I)+D * K A P(T I, D I+1))+$

$1 T *(1,-5) * K A P(T I+1, D I)+D * K A P(T I+1, D I+1))$

SAPPAEEXP(2.3026*<APP)

IF (TE. OE. 2000.) RETURV

; ** DUST PLUS GAS JPACITIES

$T=(2000,-T E) * 0.002$

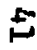
KAPPA-T *OUSTK+ $+1 .-T) * K A P P A$ RETURV

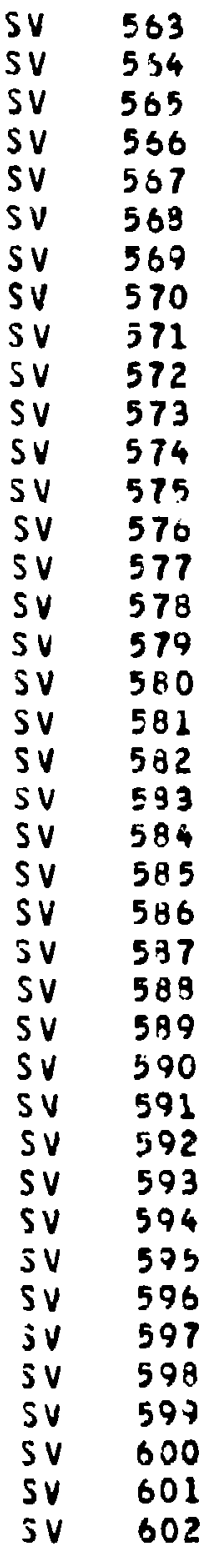


CDMMOY /AI/J3AR,JPLOJOZ,JPG, OT,T,N,NOT,FMASS,FMJM,ET,EINT,NY,LFILMSV OOO COMHJN $/ A \geq /=4(200), \times(200), U(200)$, MUVISC $(200)$

COMMJY $/ 31$ EI(200), E(200), OTIAX,VLAM,JTK

COMMON 141 RHOI20OI

COYMOY IA15I REV(200), CVD(200),R2DR(200), TNOT4

DATA PR/1.0I

$C * * *$

6 ** : SOYPUTE EONDUCTIVITY CND AND VISCTSITY YUVISC.

$C * * *$

$30 \quad 10 \quad j=3,7 P L$

TEY EEI IJ) RRCV(J)

MUVISE $(J)-7 \cdot 15 E-05 * S$ JRT(TE,T)

TEM $=0.5 *(T E M+E I(J-1) * R(V(J-1))$

ROE $=0.5 *(2 H J(J)+R H J(J-1)$

$\triangle A=R D E * T E M * K A P P A(Z D E$ OTEM)

TGRADE2。*(RCV(J)*EI(J)-EL(J-L)*RUV $(J-1)) * R 2 O R(J)$

CND(J)=3.023日3E-34*(TE.4*\#4-TNJT4)/(1.-4.*TJRAD/(3.*AA) )*AA)

$\therefore \quad C N D(J)=3.02383 E-06 * T E M * T E, 4 * T E M /(R J E * R A P P A(R J E, T E M I)$

CNDIJ) $=0.5 *(H J V I S Z(J)+4 J U I S C(J-1)) / P R$

10 CJNTIVJE

MUVIS: (1) * MUVISC (2)

MUVISE(JPI)AMUVISC (JPI)

CND $(J P I)=C N D(J P I-1)$

SND $(J P I+1)-C N D(J P I)$

RETURY

END

SUBROUTINE DECB(IER)

ZOHMJN ML, MU,B(770,1S), N, VOI1,IP(770)

LU OECOMPJSITIJN JF BAVO YATRIX A. L LJOP*A.WHERE $P$ IS A PERMUTATION MATRIX, L IS A JNIT LJWER TRIANGULAR MATRIX, ANO U IS AN UPPER TRIANGULAR MATRIX.

N CRDER OF YATRIX.

B - A $(2 * M L+4 J+1)$ ARRAY CONTAINING THE MATRIX A ON IVPUT

ANO ITS FACTOREO FORM ON OUTPUT.

ON INPUT, B(I,K) (I.LE.L.LE,V) CONTAINS THE $<-T H$

JIAGQVAL OF $A$, OR A $(I, J)$ IS STJRED IV $B(I, J-I+M L+1)$. 


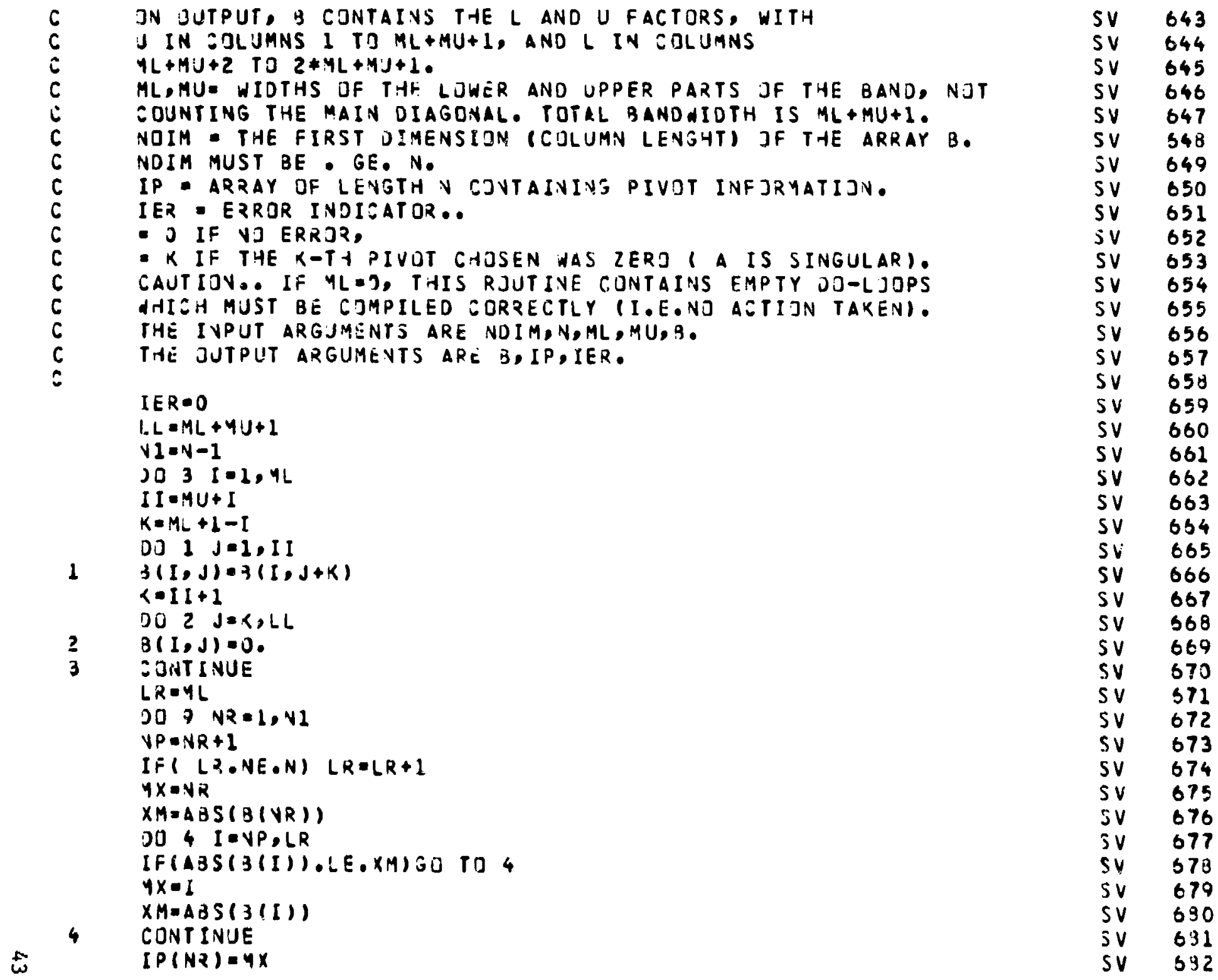




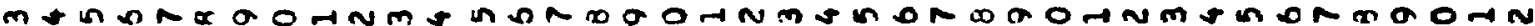

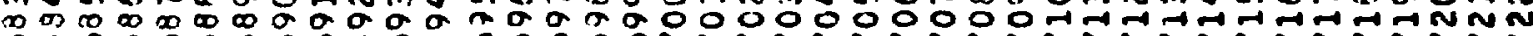

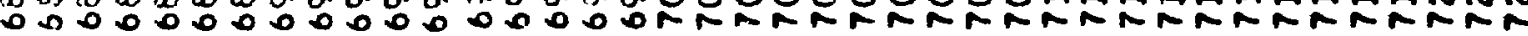

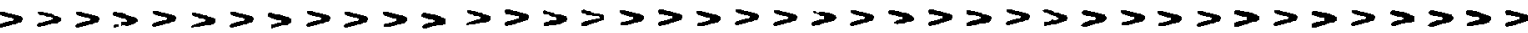

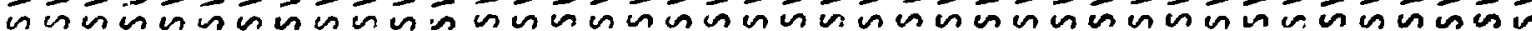
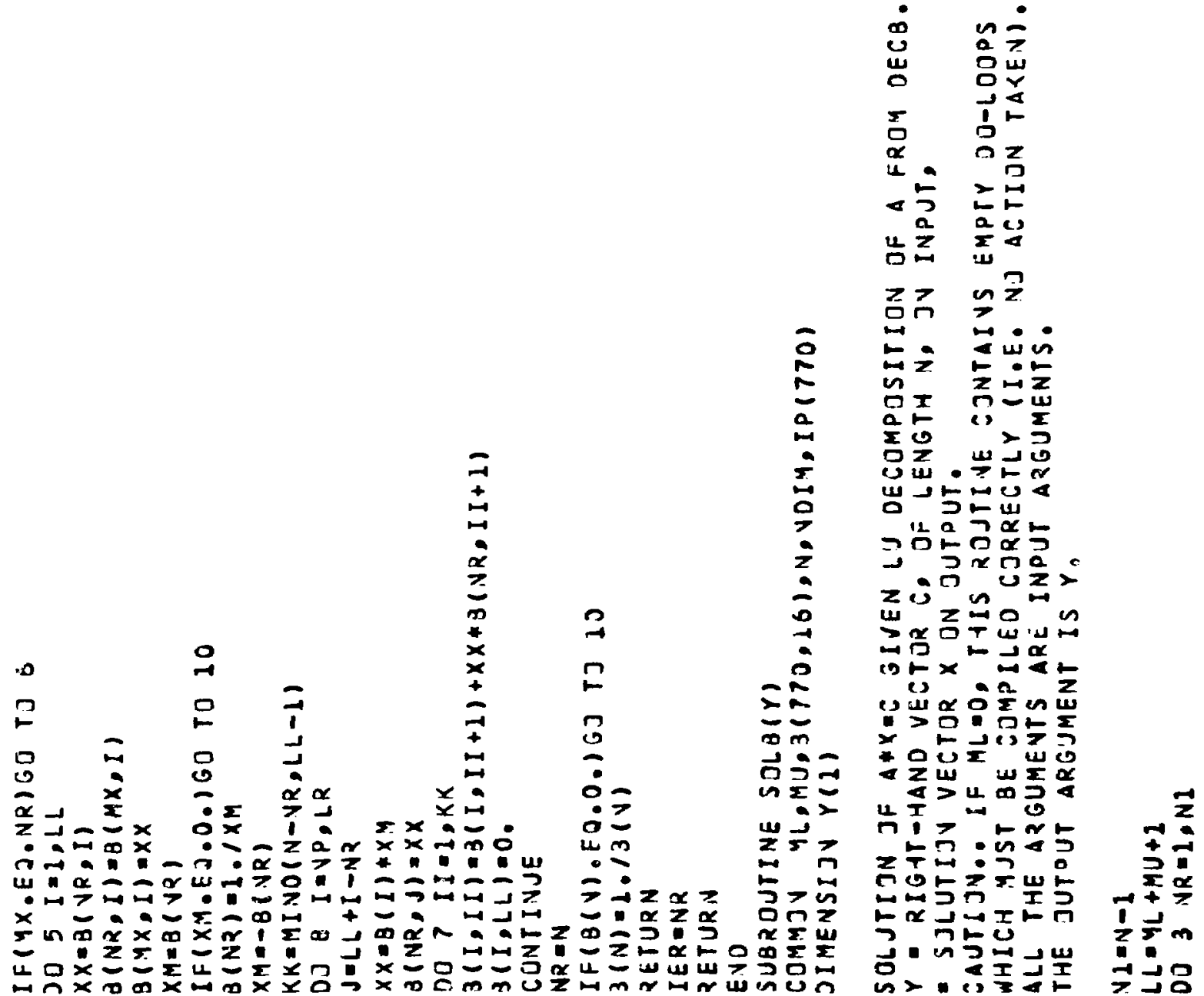

no 0 - 0 


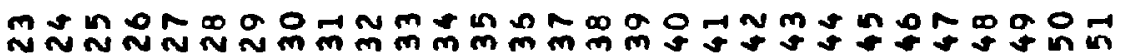

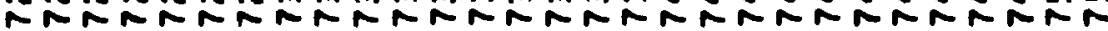

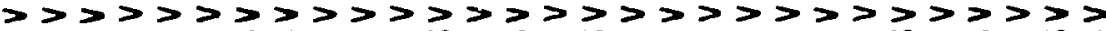

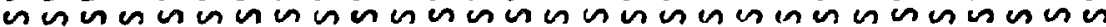

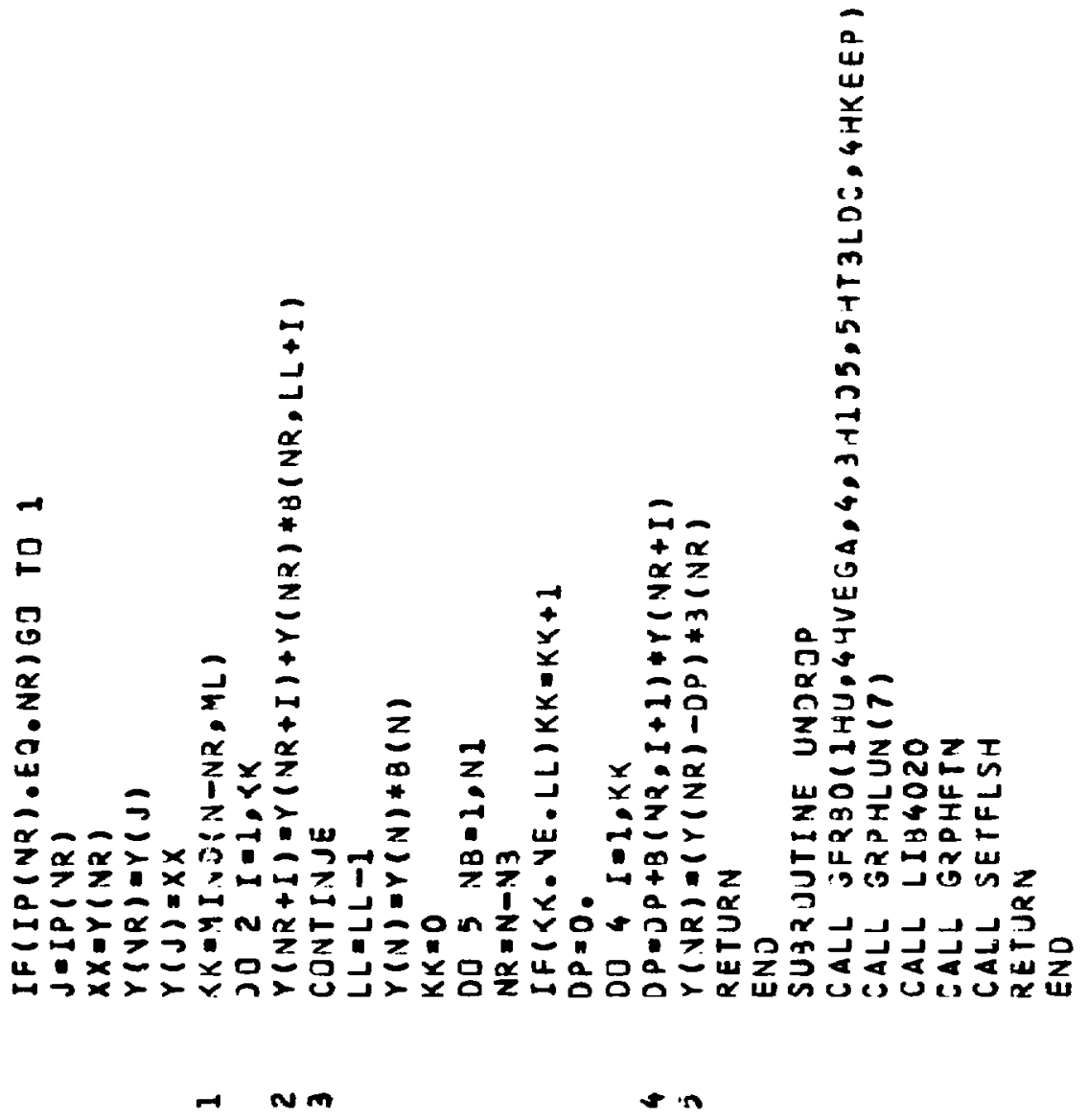




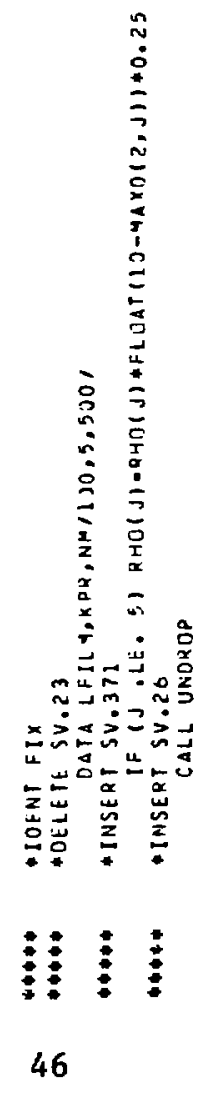




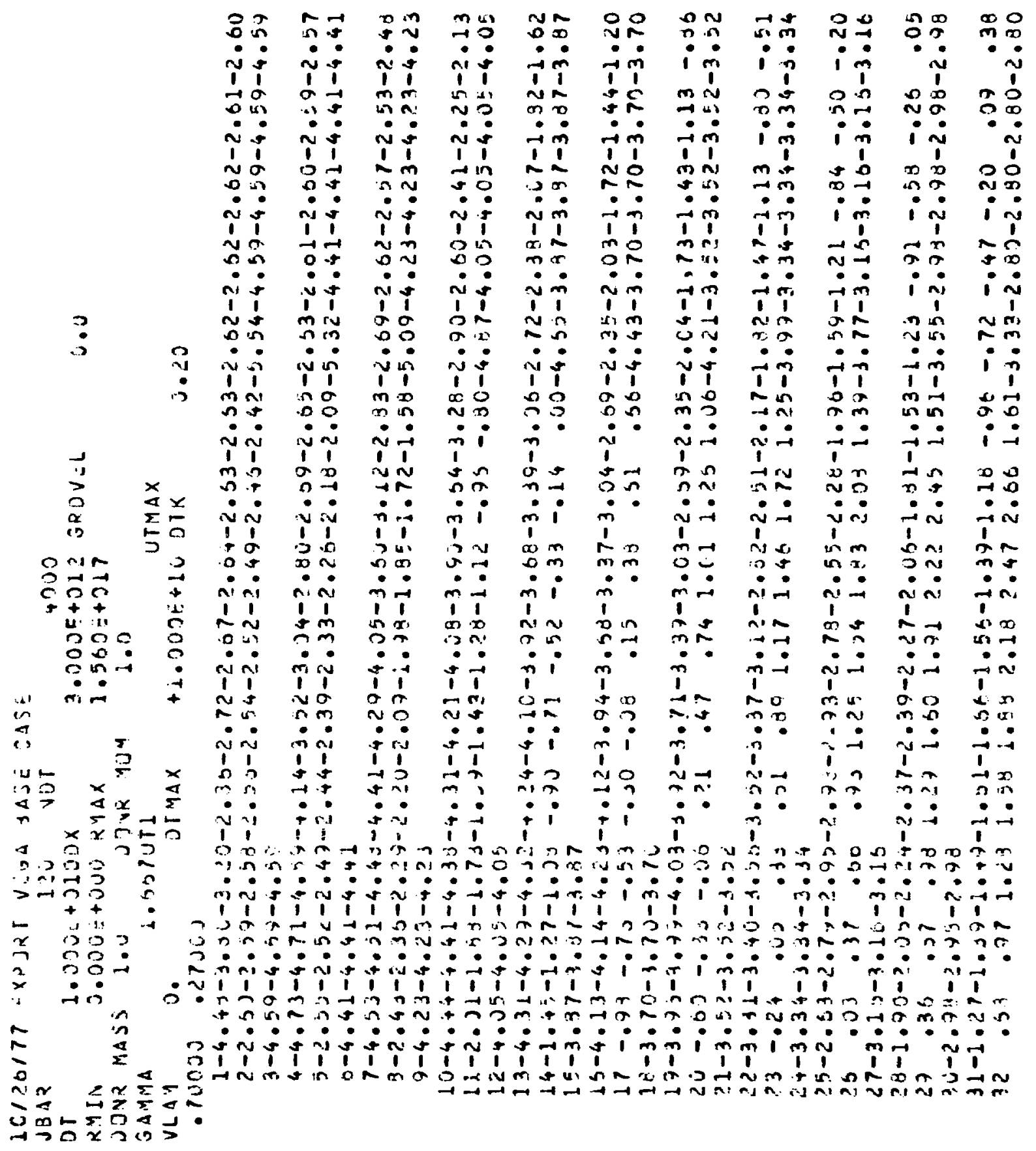

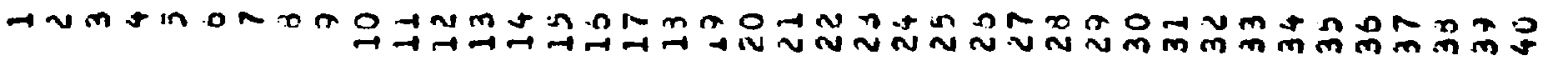




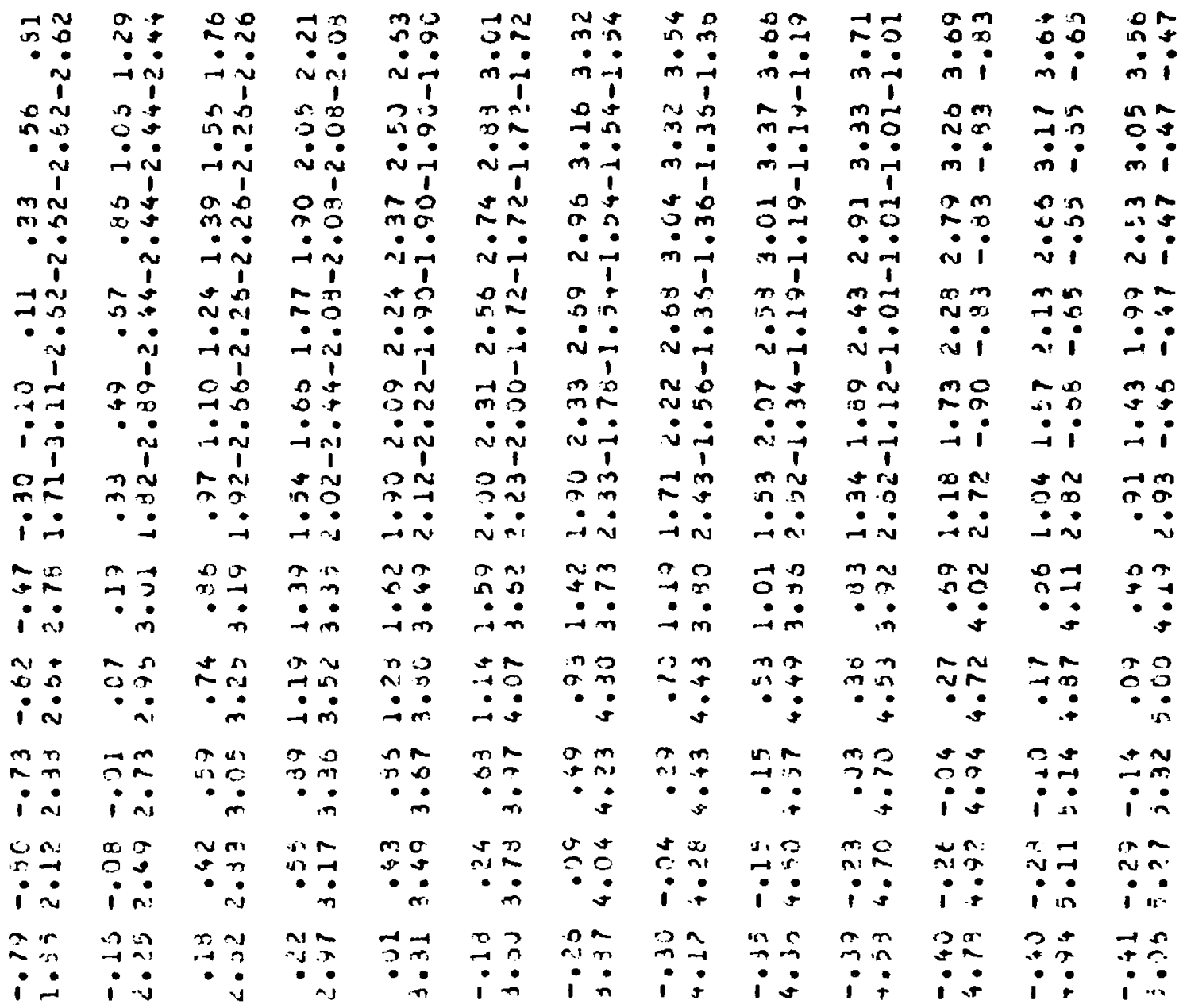

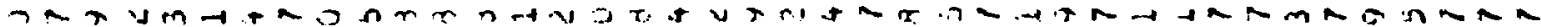
tia i i i i i i i 1 1

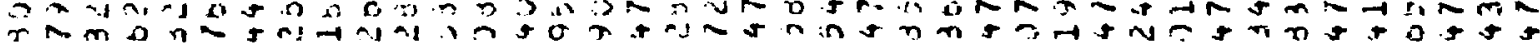

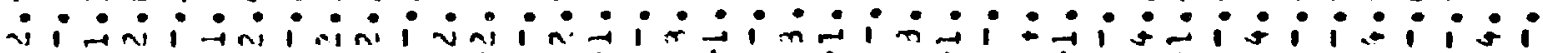

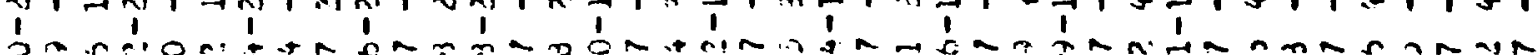

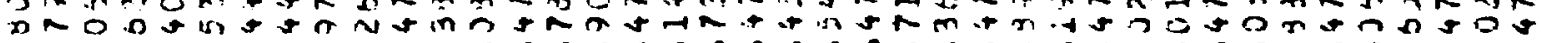

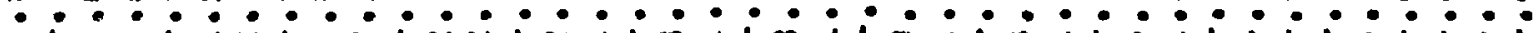

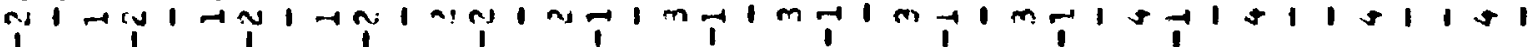

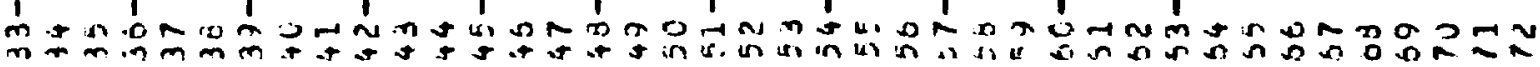




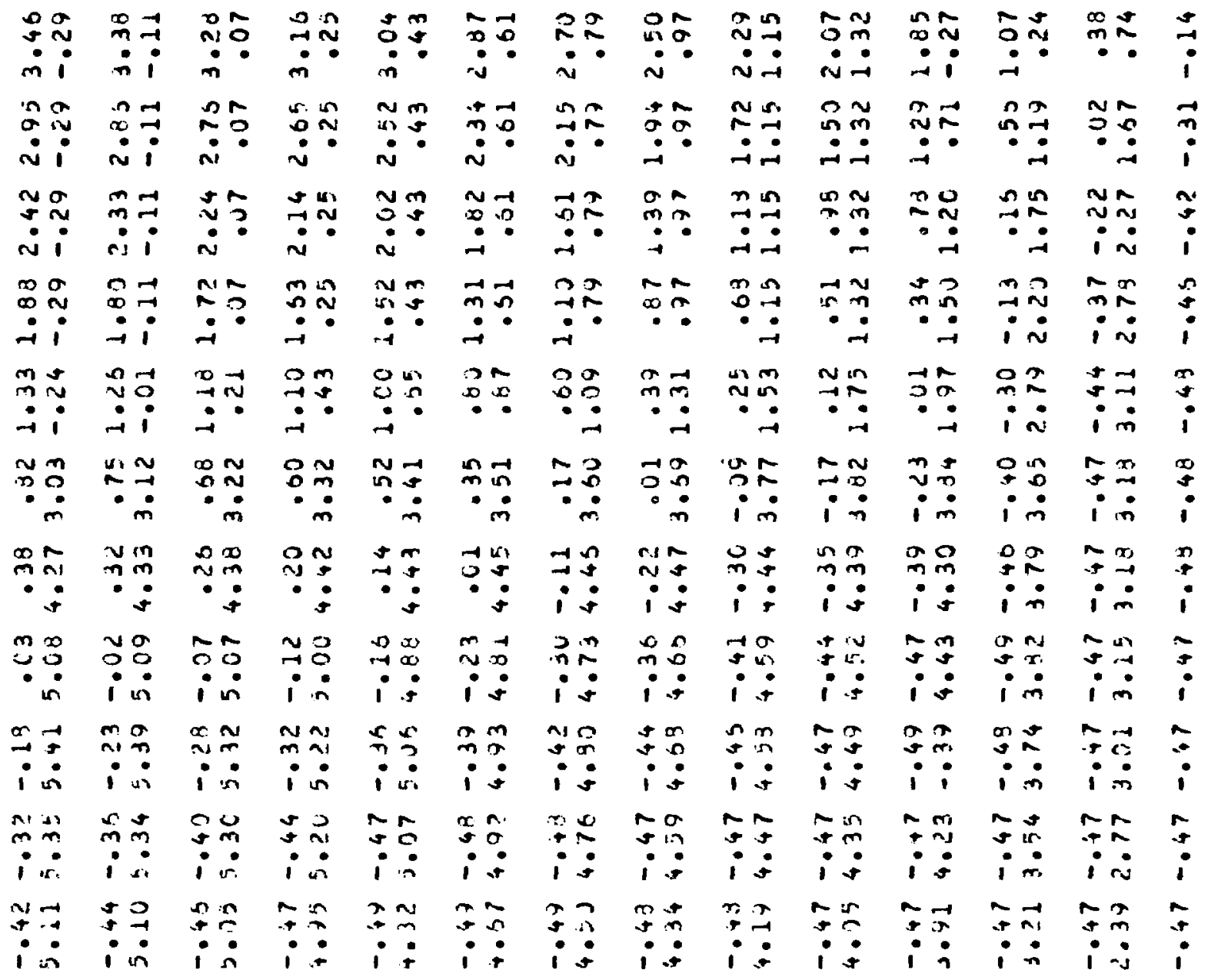

几 $\sigma$ -

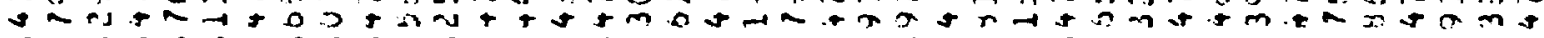

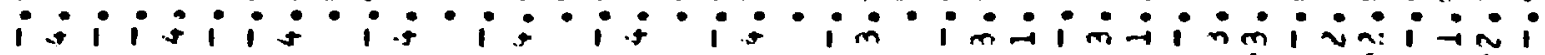

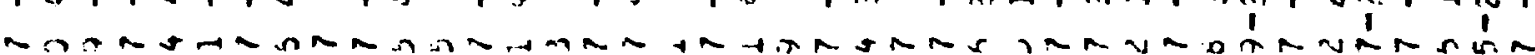

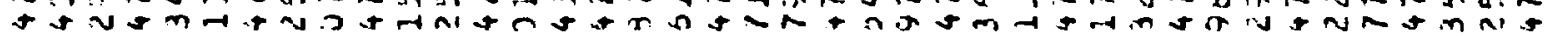

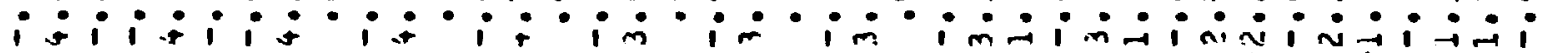
N w r

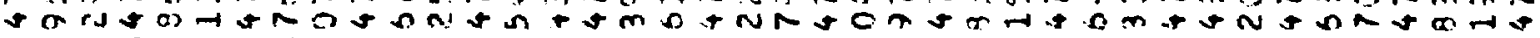

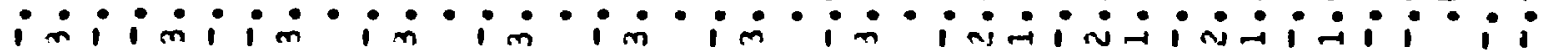

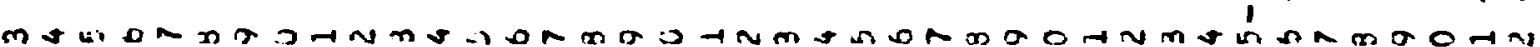

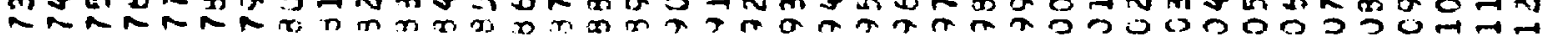

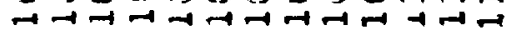




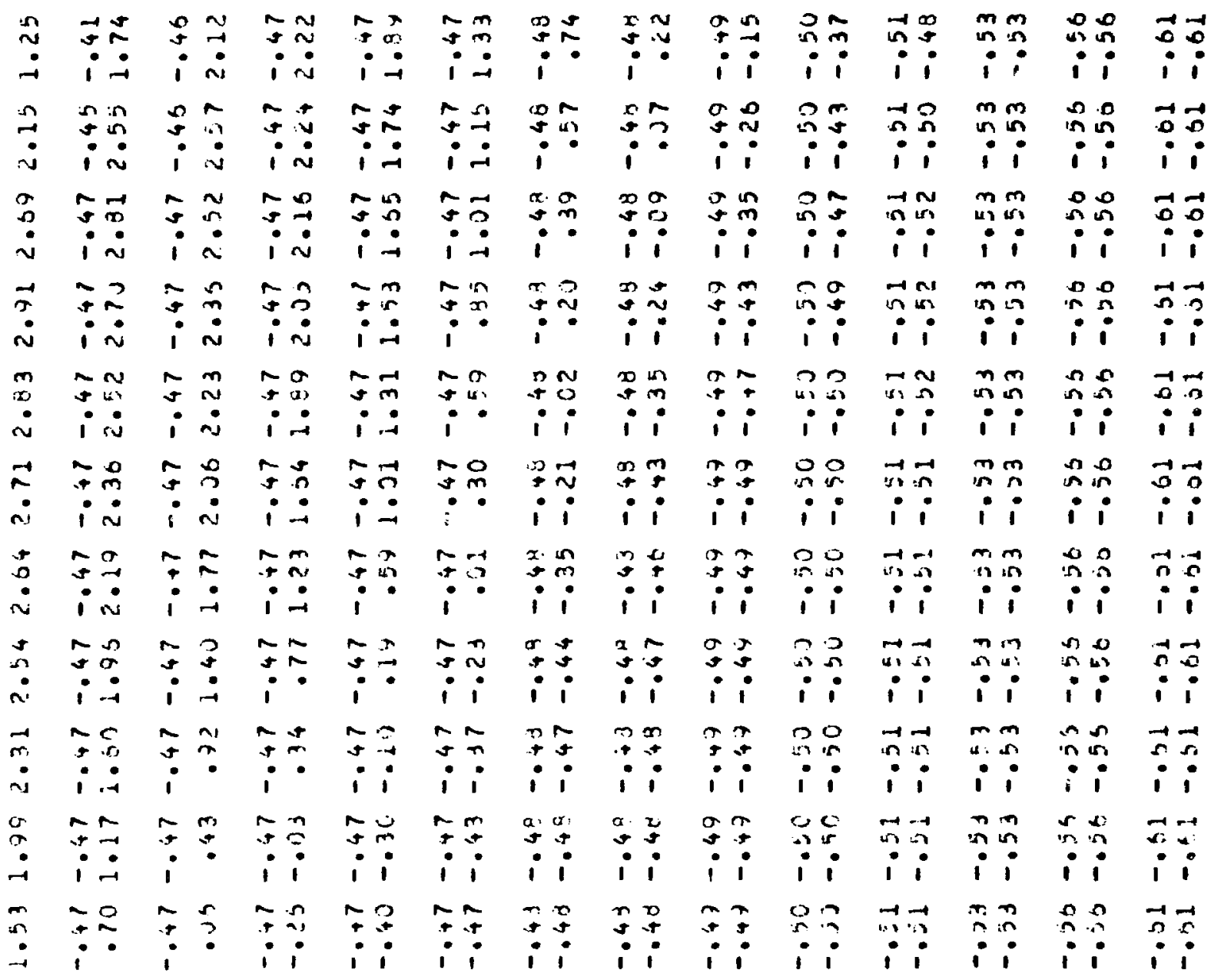

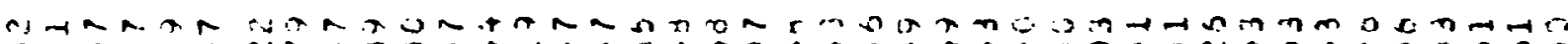

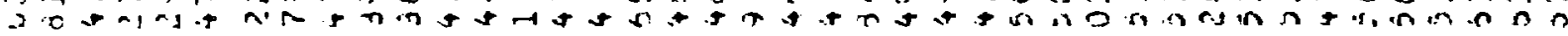

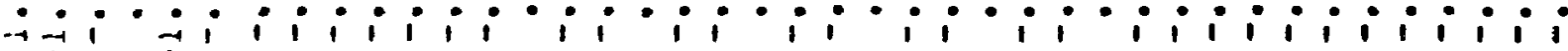

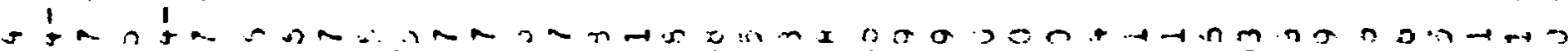

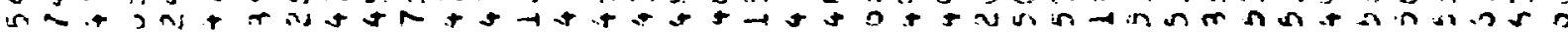
i i i i i i i i i i-i i i i

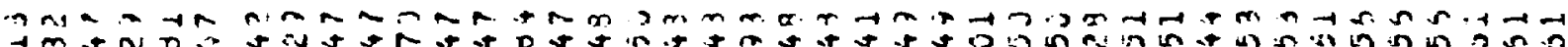

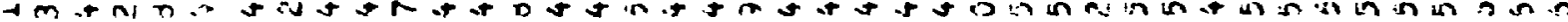

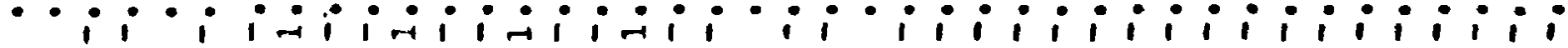

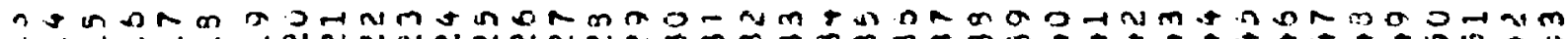

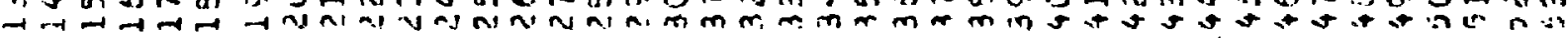

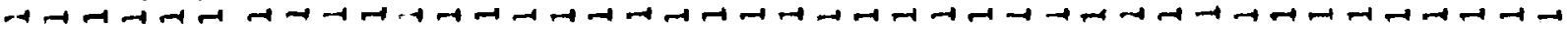




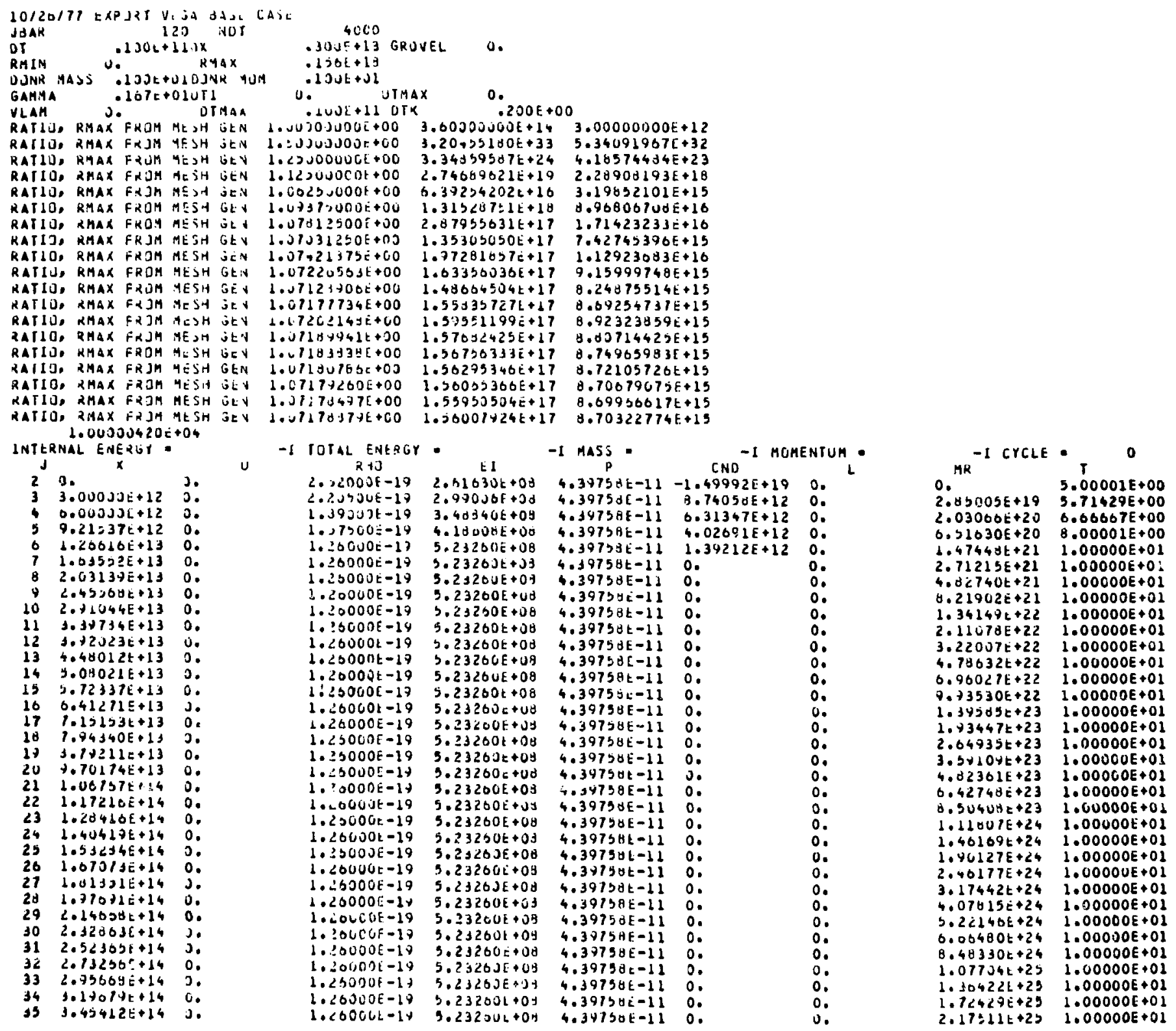




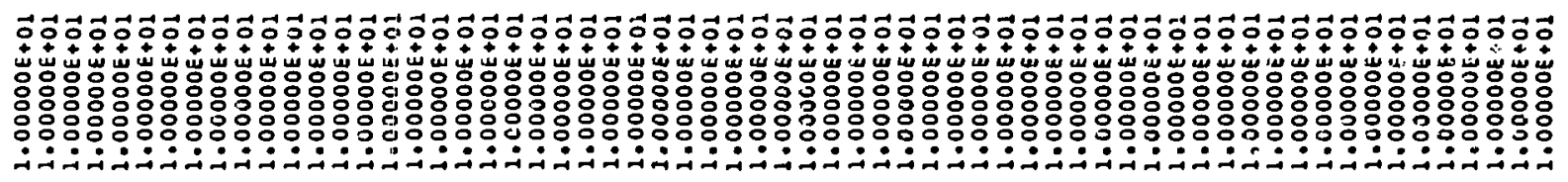

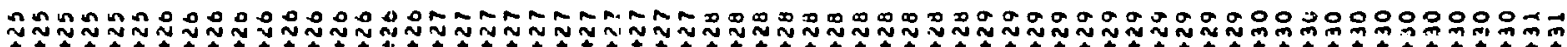

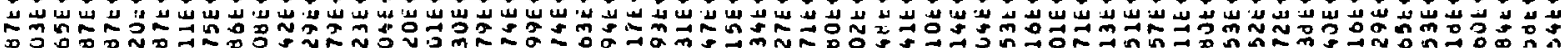

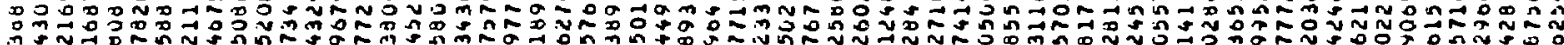

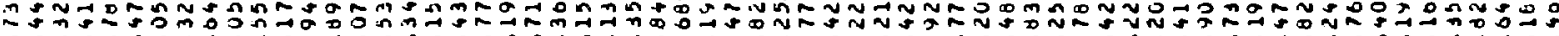

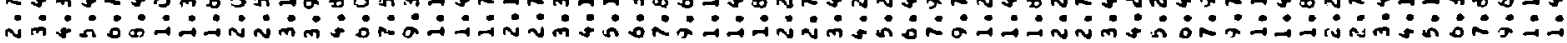

ذأهذهة

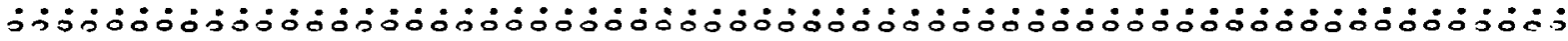

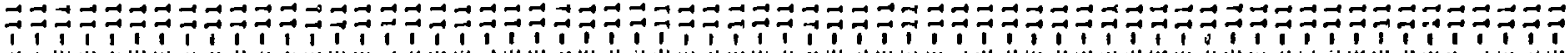

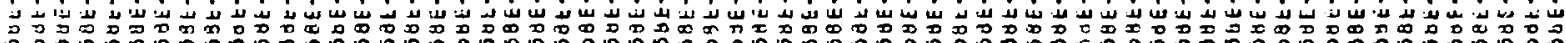
N $n$ n

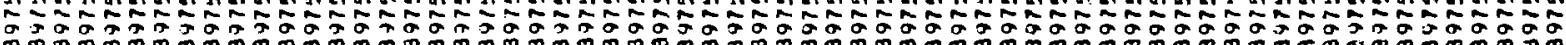

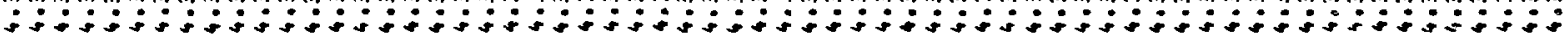

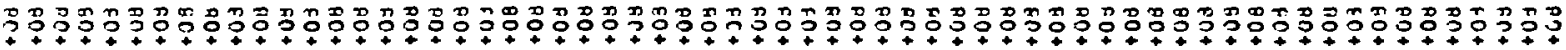

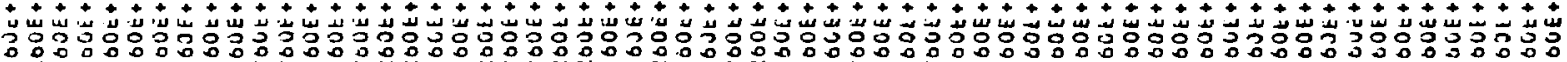

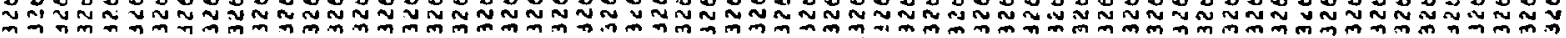

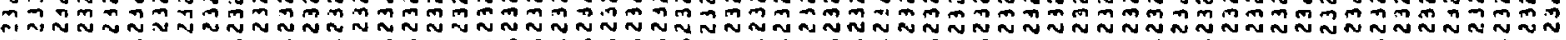
:

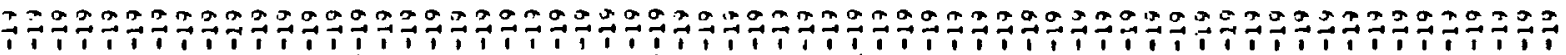

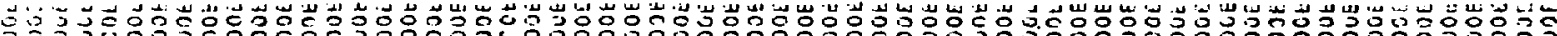

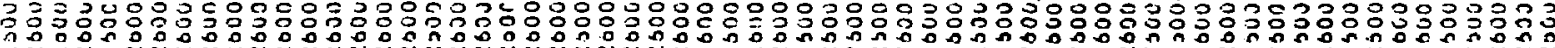

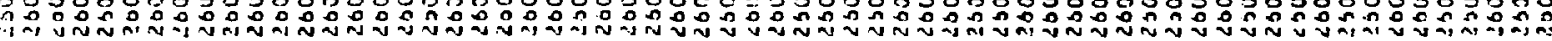

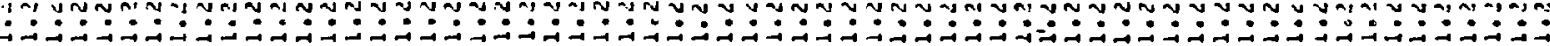

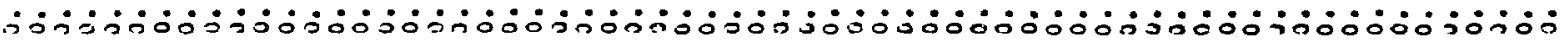

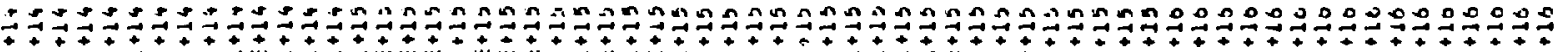

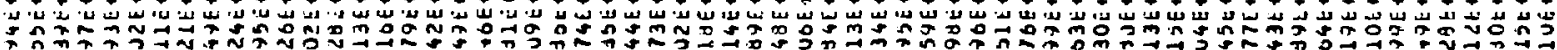

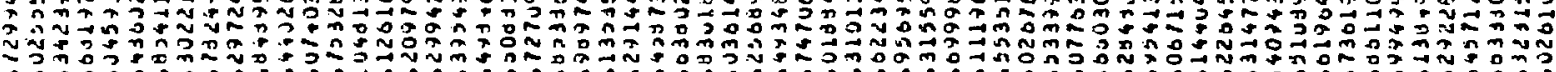

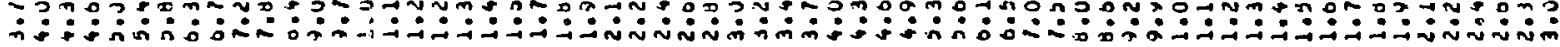

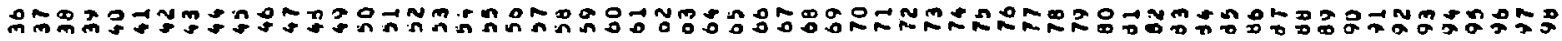




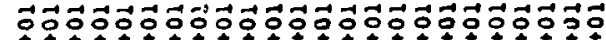

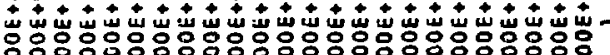

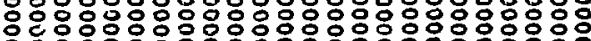

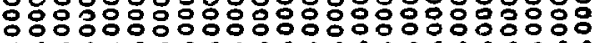

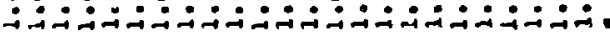

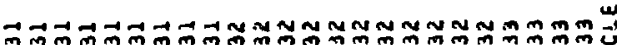

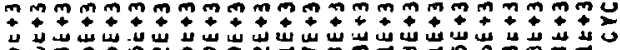

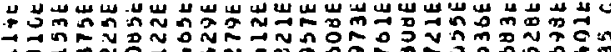

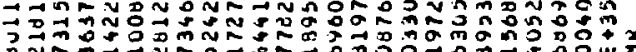

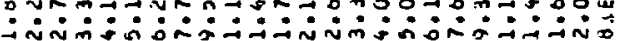
$\operatorname{Anc}$

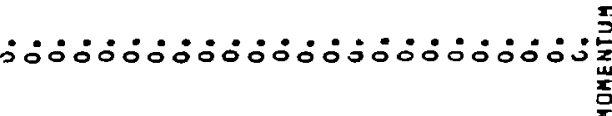

\section{in}

+ to

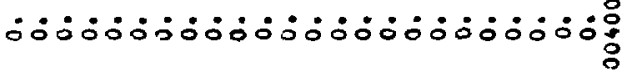

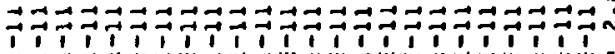

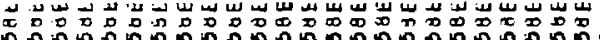

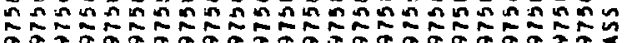

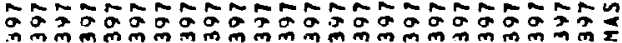

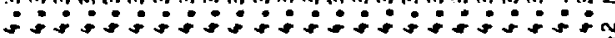

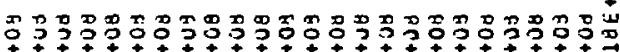

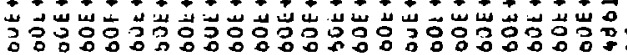

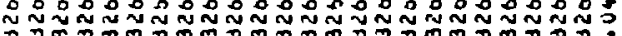

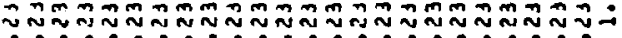

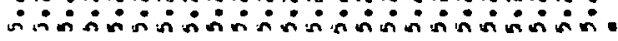

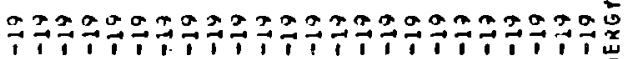

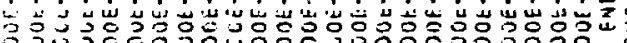

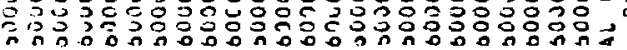

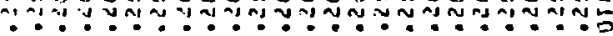
○鿑

?

苛.

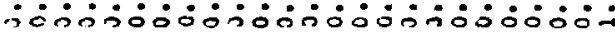

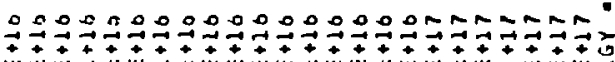

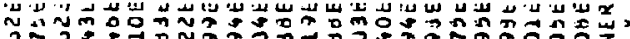

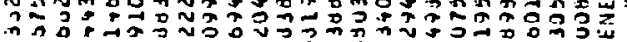

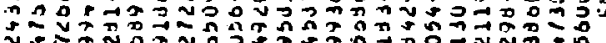

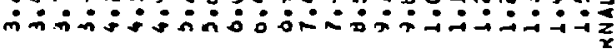

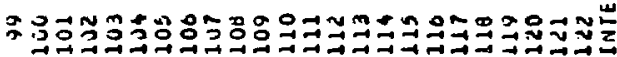

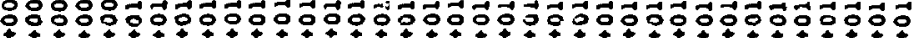

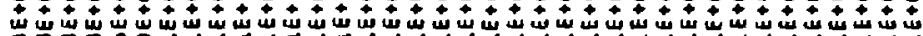
긋 m

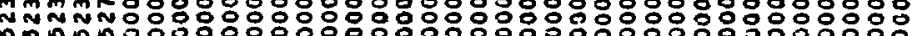

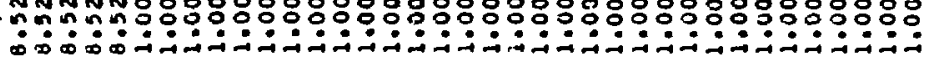

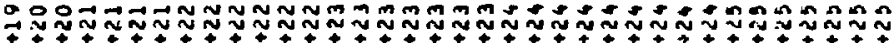

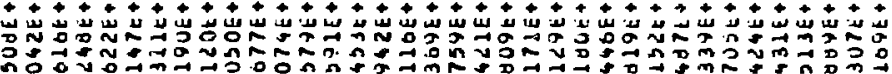

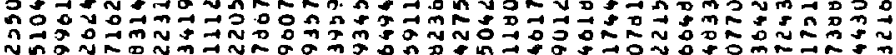

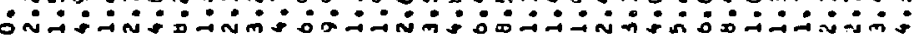

$\because \rightarrow 0$

$\because \underset{7 \rightarrow}{\rightarrow \rightarrow}$

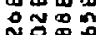

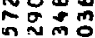

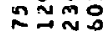

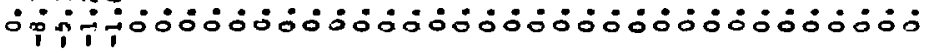
onNNN

$\overrightarrow{2} \div \overrightarrow{+}+7$

N

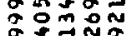

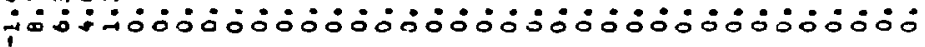

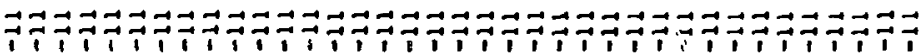

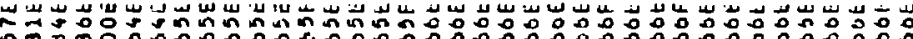

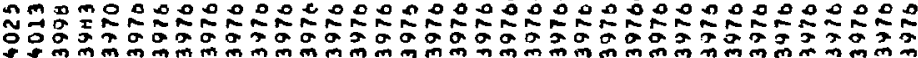

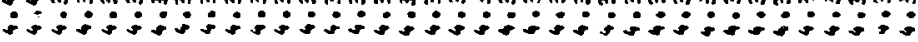

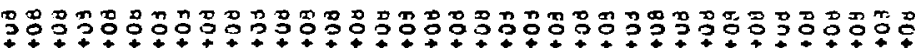

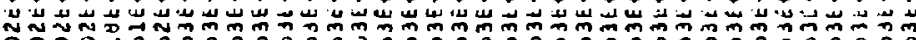

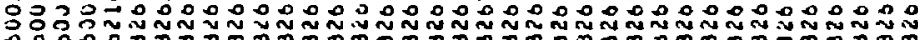

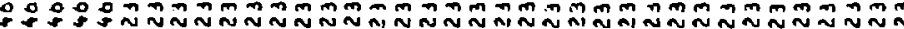

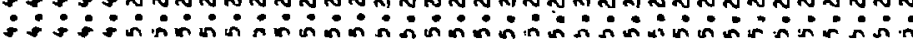

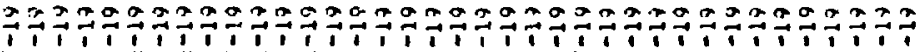

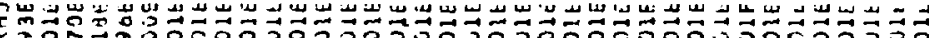

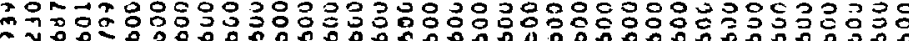
v. 워

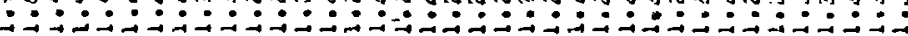

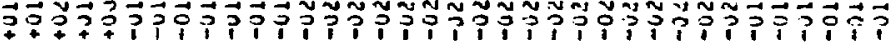

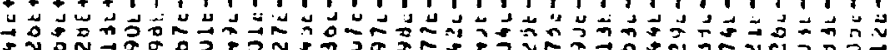
5

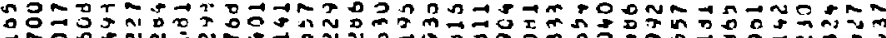

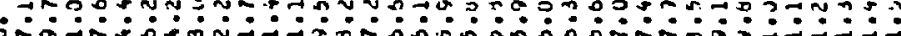

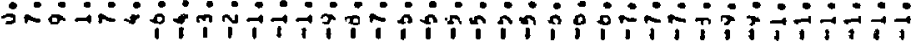

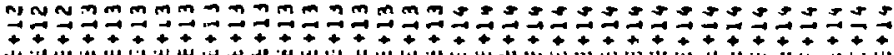

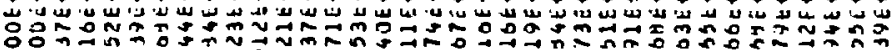

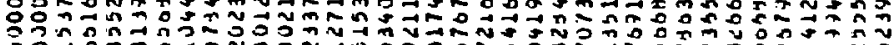

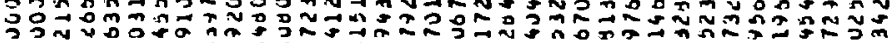

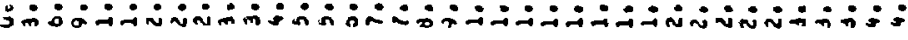

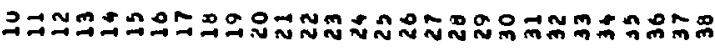




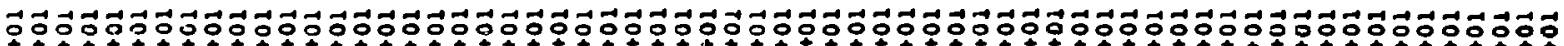

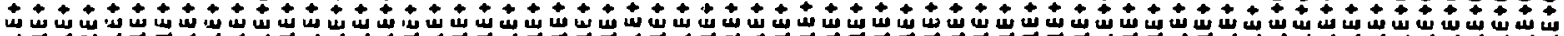

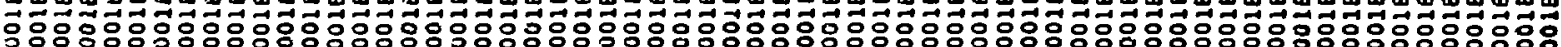

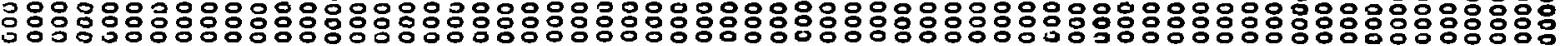
-

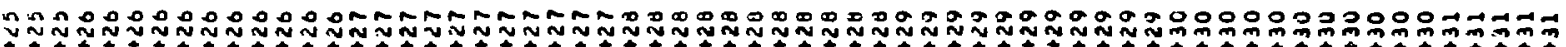

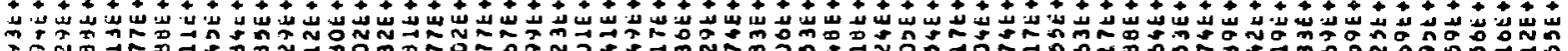

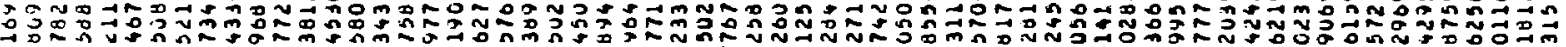

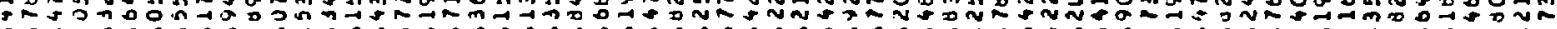

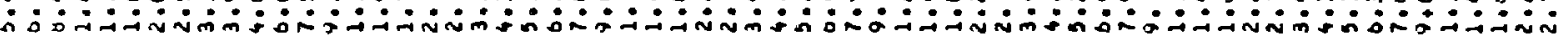

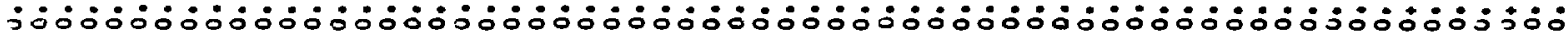

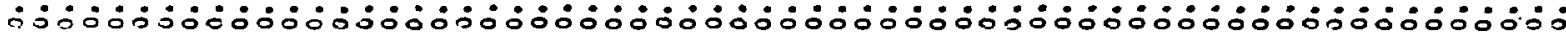

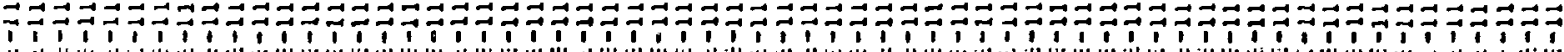
ت

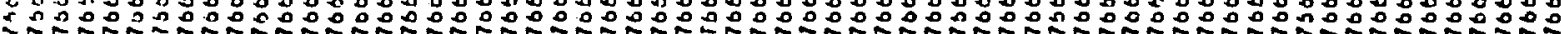

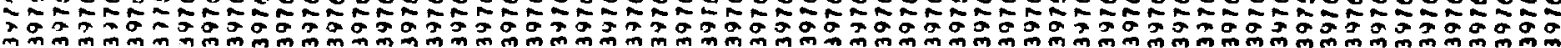
: $:$ : : : $:$ :

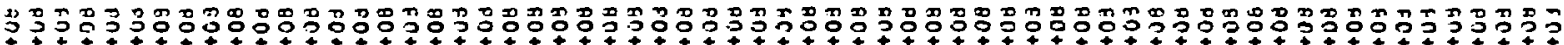

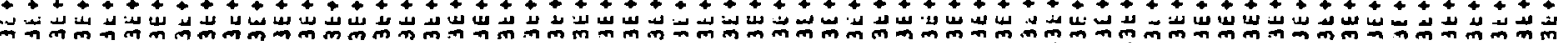

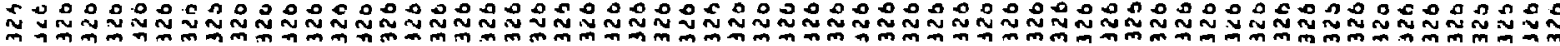

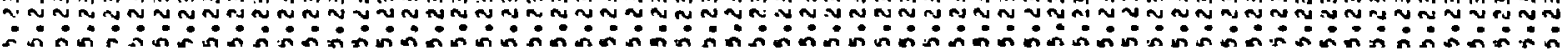

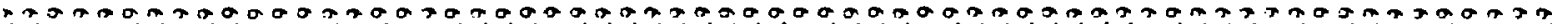

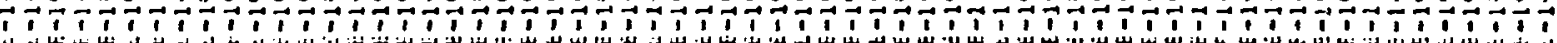

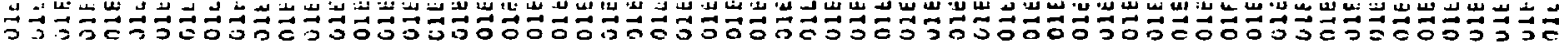

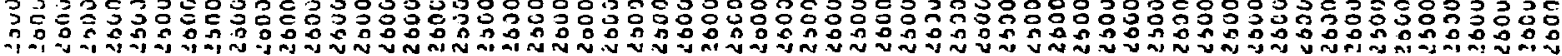
:

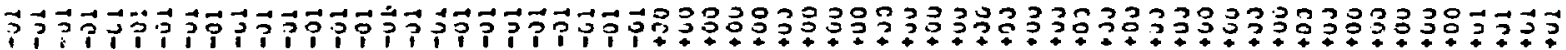

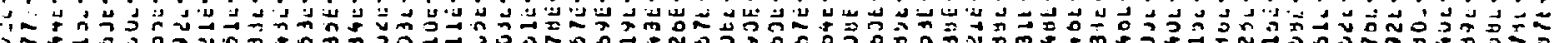

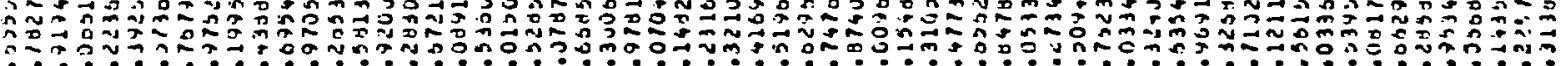

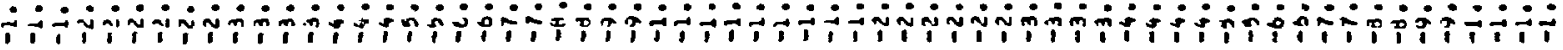
to

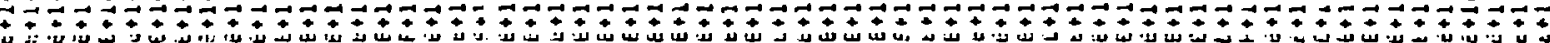

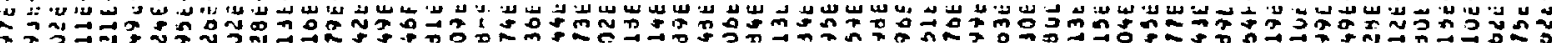

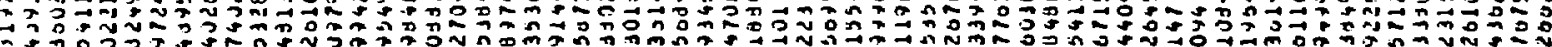

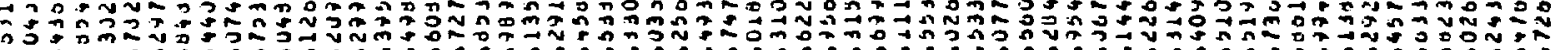
:

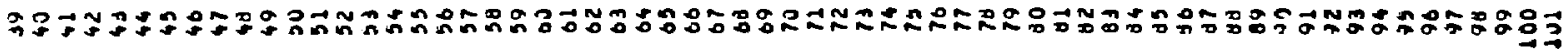




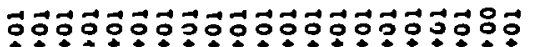
ثاثن 영ㅎㅇㅇㅇㅇㅇㅇㅇㅇㅇㅇㅇㅇㅇㅇㅇㅇㅇㅇㅇㅇㅇㅇㅇㅇㅇㅇㅇㅇㅇㅇㅇㅇㅇㅇㅇㅇㅇㅇㅇㅇㅇㅇㅇㅇㅇㅇㅇ

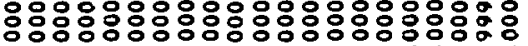

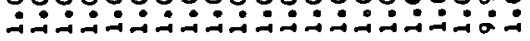

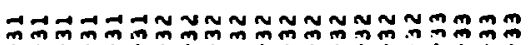

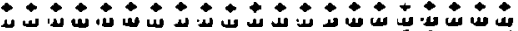

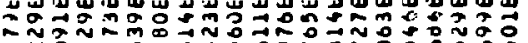

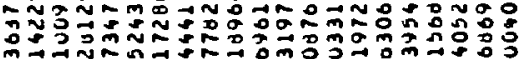

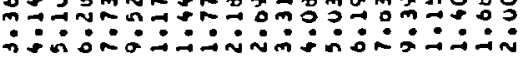

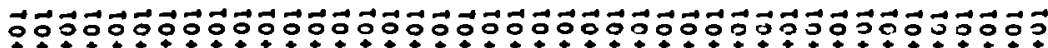

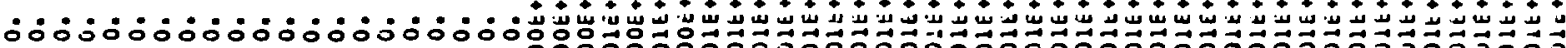

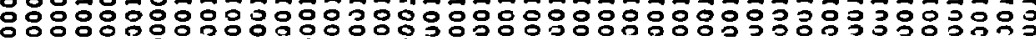

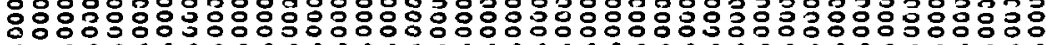

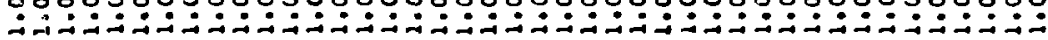

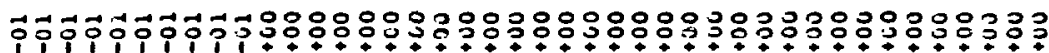

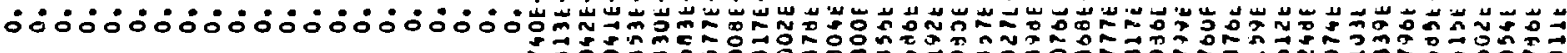

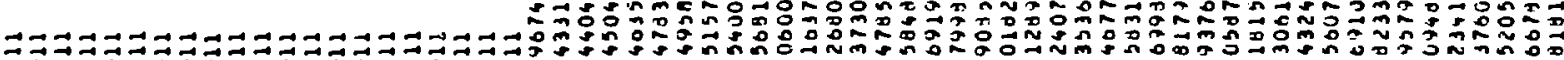

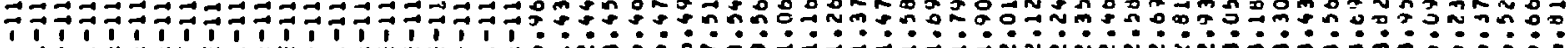

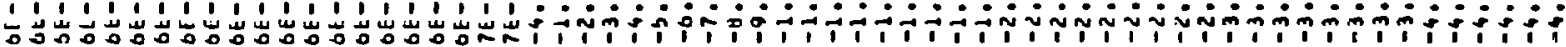

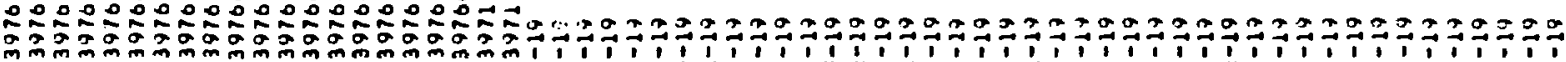

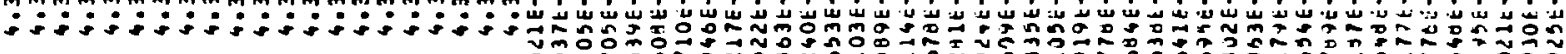

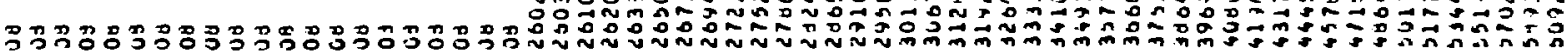

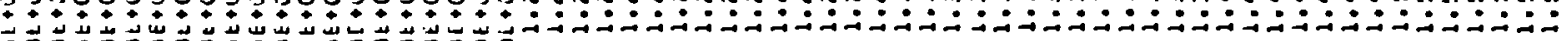

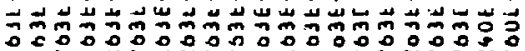

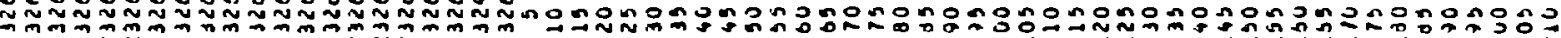

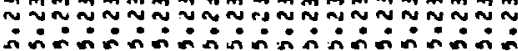

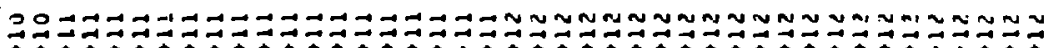

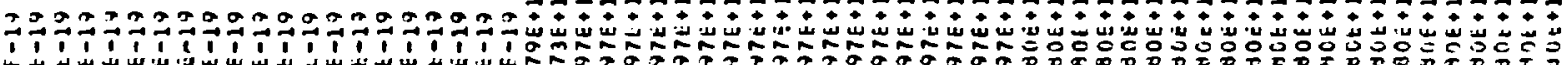

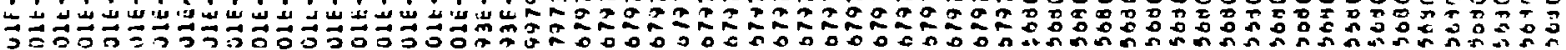

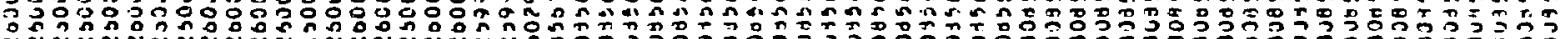

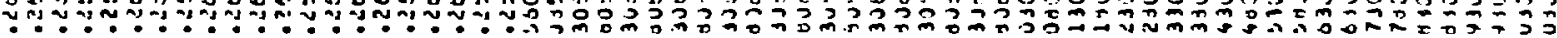
-

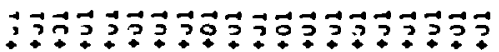

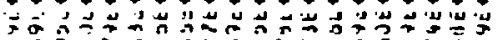

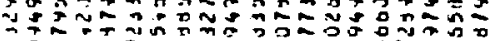

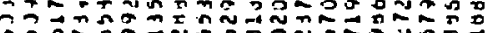

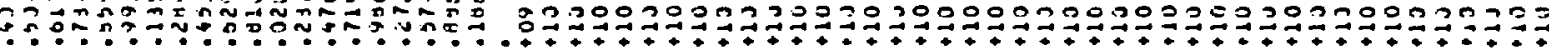

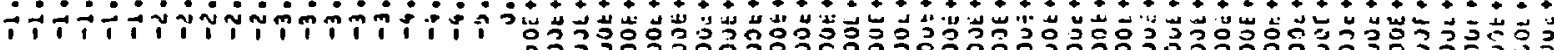

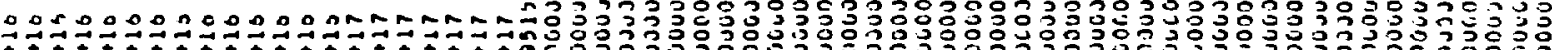

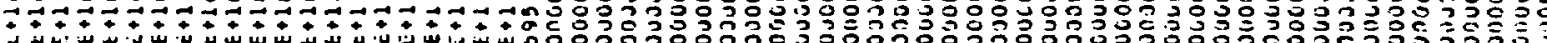

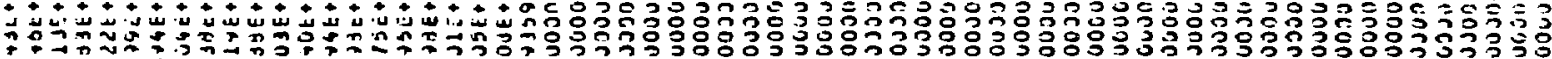

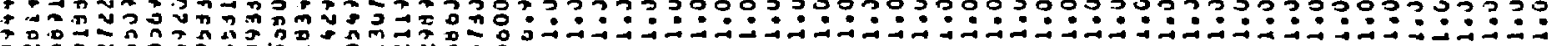

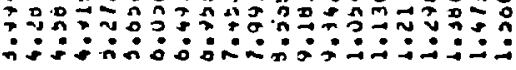

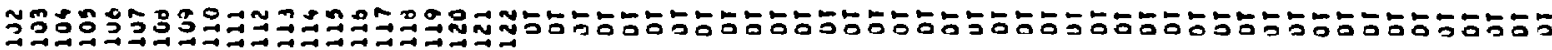




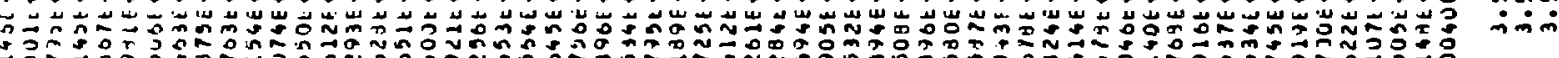

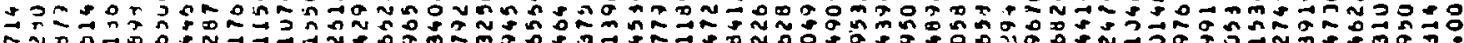

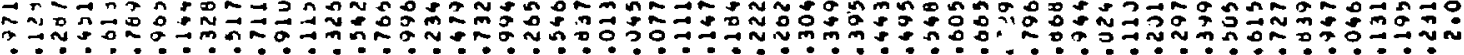

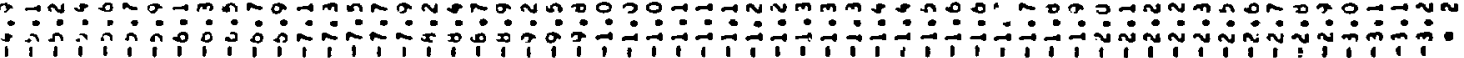

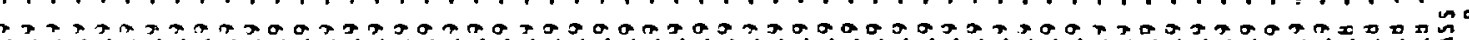

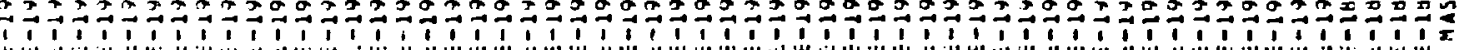

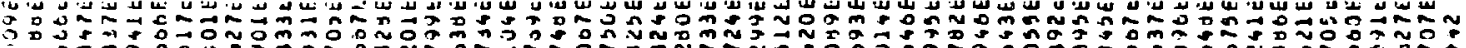

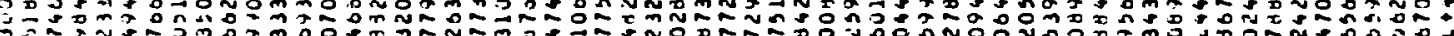

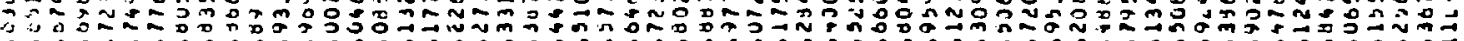

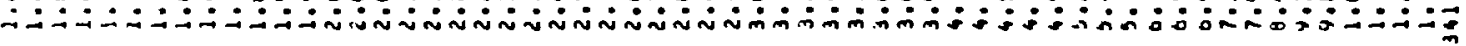

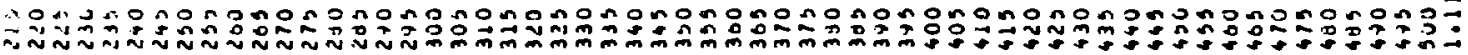

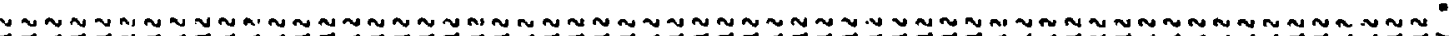

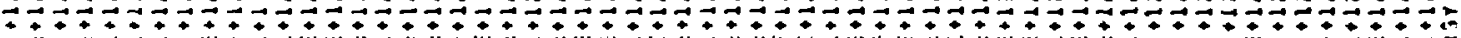

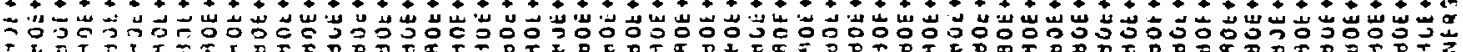

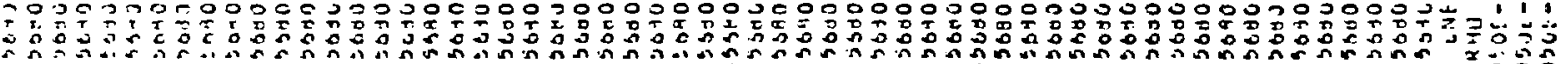

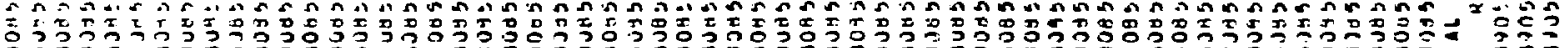

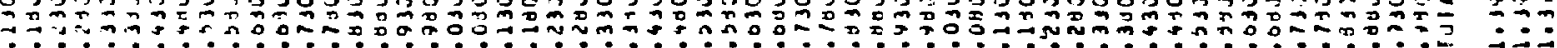

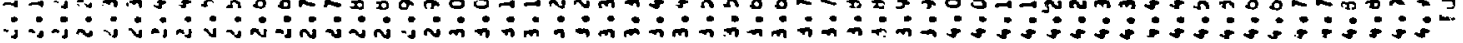

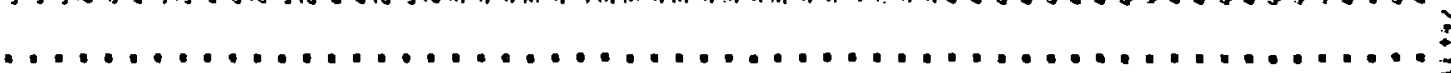

-

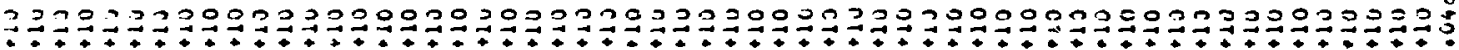

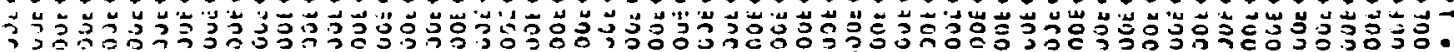

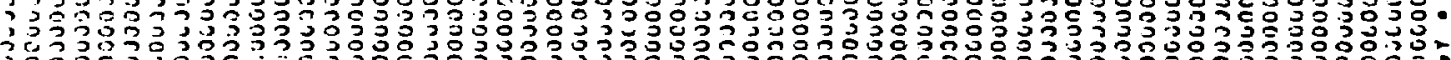

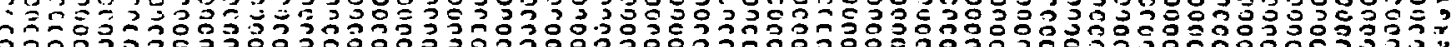

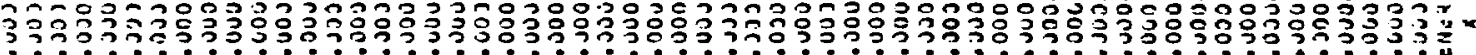
过

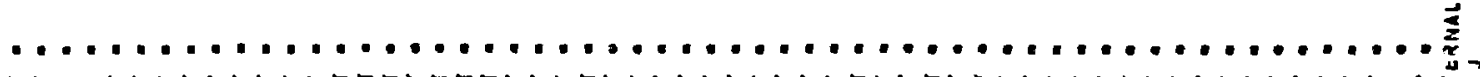

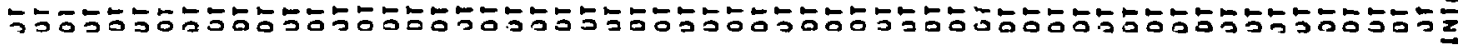




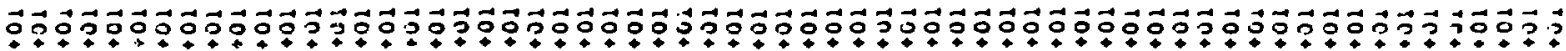

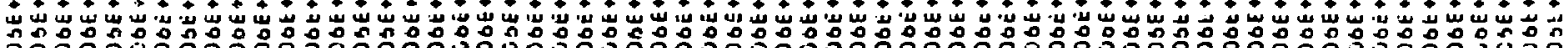

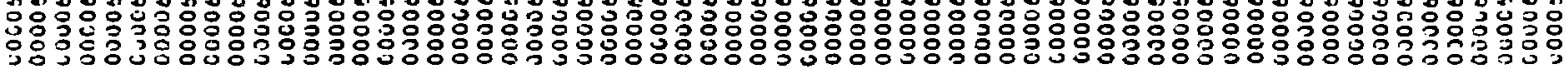

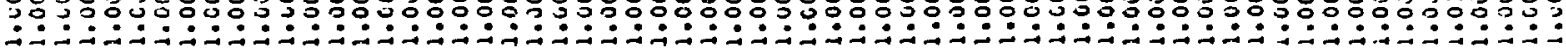

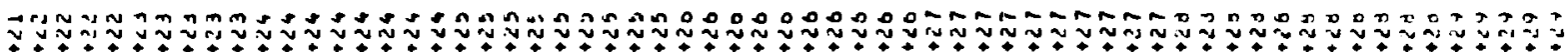

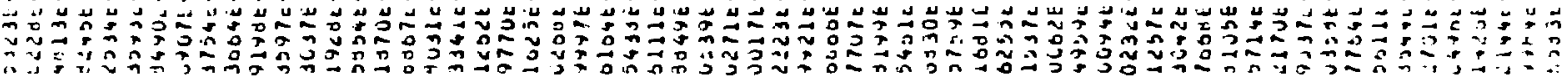

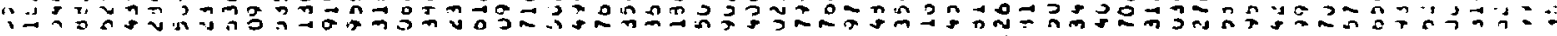

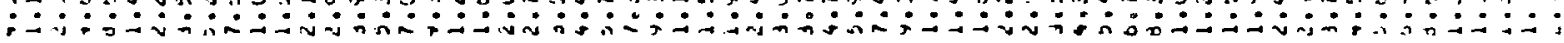

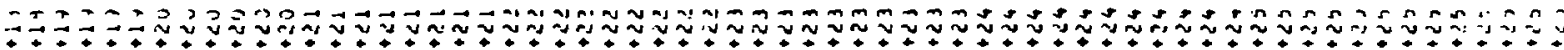

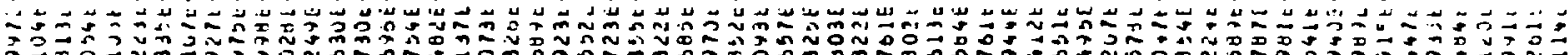

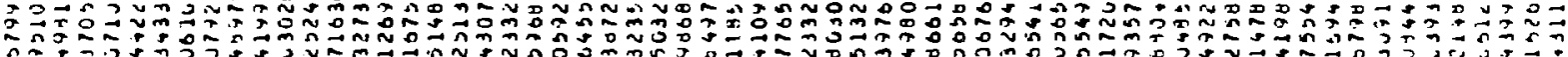

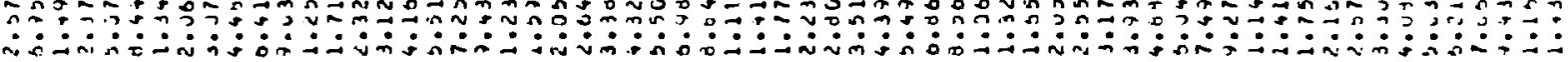

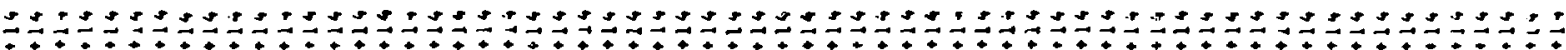

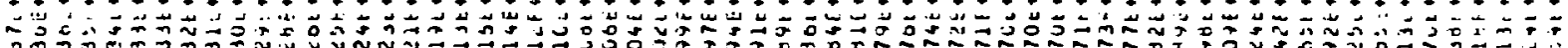

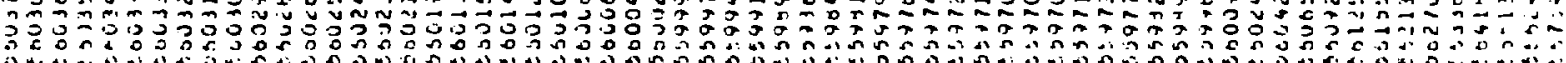

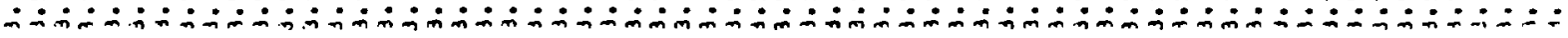

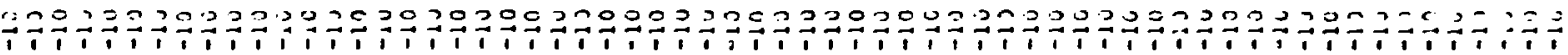
$=0$

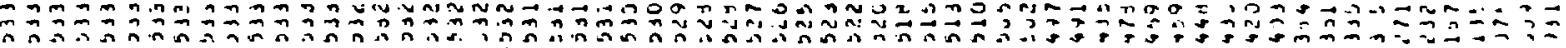

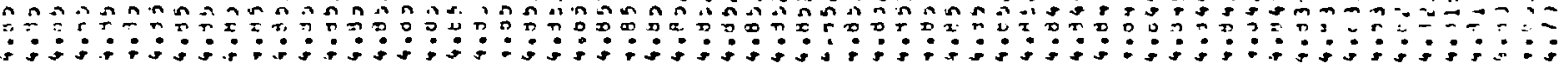

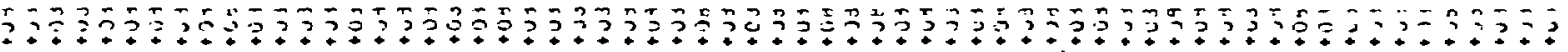

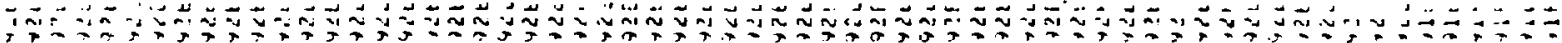

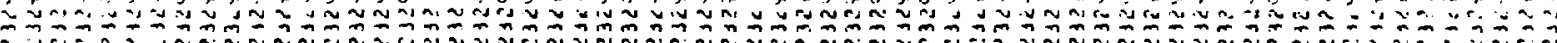

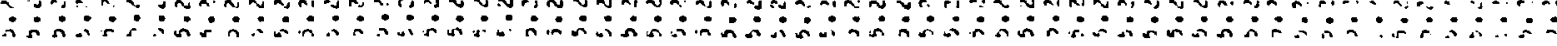

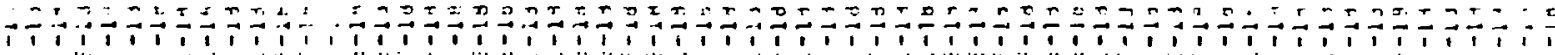

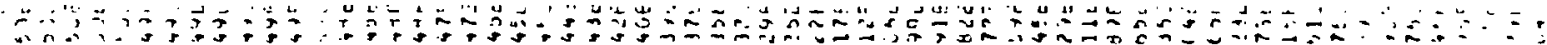

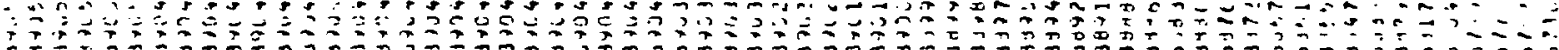
:

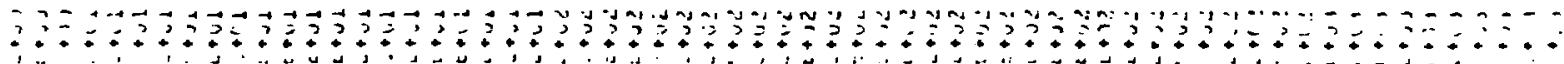

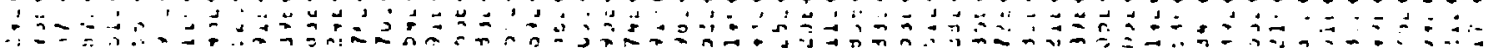

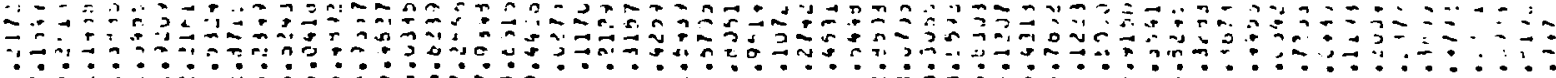

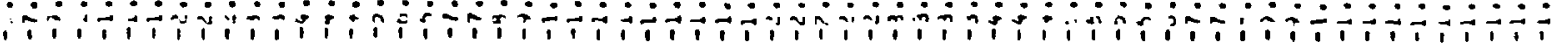

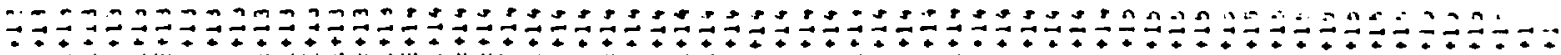

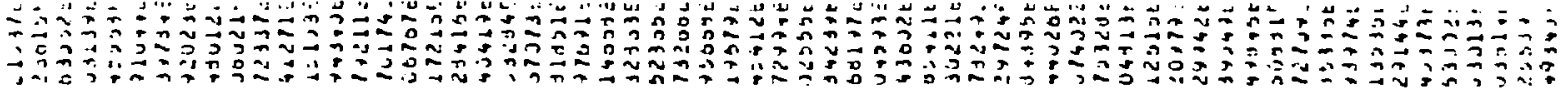
:

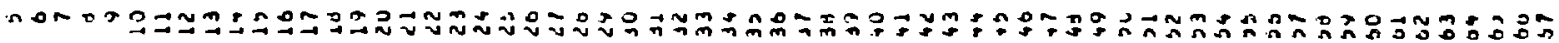




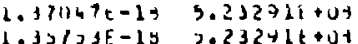

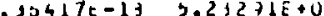
1.,., $032 t-13 \quad 9.2+2+11+1$

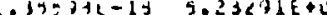
$1.12101 t-13 \quad 5.23241 E+0$ $3.23271 t+0$
$11405-13$ DJ110.E-1J $5.232+1 t+0$ $1.12340 E-19 \quad 5.23241 f+U$ $1.3140 t t-15 \quad 5.23240 t+0$ 1.103วTE-13 $2.232+3 t+03$ $1.2+17 \pm=-10 \quad 5.21240 t+00$ 1.27MG7E-10 3.232 YUE+0d 1.203bIE-1J 5.232YUE+UA $1.27101 k-18 \quad 3.23289 k+00$ 1.2203SEAIY $5.21289 E+U$ 1.2UHC9E-10 $3.2328+t+0$ 1. $10530 E-10 \quad 5.23203 E+0$ $1.113460-10$ a. $33200+00$ 1. $10310 E-15 \quad 3.23247 L+0$ $1.0714 \forall E-16 \quad 5.232 d 7 E+O$ 1.J3743E-13 $3.23286 E+00$

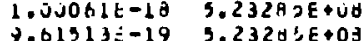
$\rightarrow .01513=-19$ 9.20312E-17 $\$ .23264<+0 J$ 3. $172 ? C E-19 \quad 5.232 H 3 t+04$ $\begin{array}{ll}9.12=07 E-19 & 5.232026+0 J \\ 1.05674 E-17 & 5.23201 E+0 J\end{array}$ 1.00474E-17 $1.1+341 E-17$ $0.22042 E-1 A$ S. $3230 L+0 J$ $0.44609 E-10 \quad 5.23270 E+0 J$ $5.47001 E-19 \quad 5.23277 t+08$ $3.3) 416 t-19 \quad 3.23275 c+00$

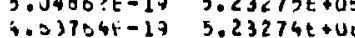
1.33704t-19 3. $71170=10$ 3.23211E+05 (1) $30 E-10$ $2.11330 E-19$
$2.41151 E-17$ $2.41151 \mathrm{t}-17$ $10,3 \rightarrow 7=-19$ 1. $350408-10$ $1.44424 t-14$ 1. $23433 \mathrm{E}-14$ 1. 0 YII 1 E-19 $4.41371 E-20$ $0.50 \leq 00 E-20$ a. $4 \sin \theta-20$ $3,+\rightarrow 3 E=20$ $\begin{array}{llll}5 .+7123 E-20 & 5.23261 E+U H & 2.09391 E-11 & 3.46242 E+1 \\ 3.17 U 16 t-20 & 3.23260 E+G O & 1.81424 E-11 & 3.46242 E+1\end{array}$ $\begin{array}{llll}3.17 U 16 t-20 & 3.2 J 260 E+08 & 1.81424 E-11 & 3.46242 E+1 \\ 3.17-11 t E-3 . J & 3.23200 E+0 J & 1.81424 t-11 & 3.46242 E+1\end{array}$ S.030 गמपOE + $3.2303 \operatorname{sen} n e+12$ $5.14053+36+12$ $\therefore, 03005050 \mathrm{col}+1$

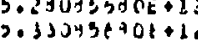
j. 3 jugsetul +1 (1) $4.697071=10 \quad 3,50310 E+14$ $4.075=0 E-10 \quad 3.389+2 t+14$ $4.05035 E-10 \quad 3,59441 E+14$ $3.60013 E+14$
$4.00014 t+14$ $4.56+00 E-10 \quad 3.60674 E+14$ 4.5533JE-1U $3.01432 E+14$ $4.46050 E-10 \quad 3.63295 t+14$ $4.41505 E-10 \quad 3.64432 E+14$

$4.29304-10 \quad 3.67210 E+14$ $4.22270 E-10 \quad 3.60894 E+16$

$4.174322-10 \quad 3.70007 E+16$
$4.03+11 E-10$

$3.96372 E-10$ 3.75629E+14

$3.86090 E-10$
$3.746170 E+14$ $3.62964 E-10 \quad 3.848016+14$ $3.50138 E-10 \quad 3.60704 E+14$ 3.30S13E-10 $3.22142 E-10 \quad 3.97071 E+14$ 1.07202t-1 $2.75406 E-10$ $2.5898 E E=10$ $2.25098 E-10$ $3.09119 \mathrm{E}-10$ $1.92779 E-10$ $1.61362 E-10$ $1.40>27 t-10$ $1.32509 E=10$
$1.19387 E-10$ 1. COOOI AE-10 $9.30413 t-11$
$8.43732 E-11$ 1.4017ite-1 3.2320

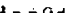
$3.23207 \mathrm{E}+0 \mathrm{~A}$ $3.23260 \dot{0}+0$ $3,2 \$ 356 E+D O$ $3.712 \$ 7 E-1$ $3.048+7 E-11$ $4.03195 t+14$ $4.09047 E+14$ $4.15450 E+14$
$4.22919 E+14$ $4.24965 t+14$ $4.46730 E+14$
$1.36020 E+14$ $4.6,773 E+14$ $4.19987 f+14$ $4.80503 E+14$
$4.97455 E+14$ $3.00450 E+14$ $3.19385 E+14$ $5.29972 E+14$ $3.40360 E+1$ $3.35473 E+14$ $3.00605 E+14$ $67905+14$ $\begin{array}{lll}3.2 \$ 2041+08 & 3.01209 E-11 & 9.40960 E+14 \\ .0232031+0 y & 3.28098 E-11 & 9.27299 E+14\end{array}$ $0.23202 t+03 \quad 2.023622-11 \quad 4.94215 t+14$ . $23202 t+63$ 2.02362k-11
$4.734311-10 \quad 3.369365+14 \quad 1.70016 E+26$ $477410 E-10$ 3.57084E+14 $2.15550 \mathrm{~T}+20$ $470<01 E-10 \quad 3.572416+14 \quad 2.00035 E+20$ $4.1674 J E-10 \quad 3.37532 t+14 \quad 3.26722 t+2$ $2.91+601-10$ $4.3008 B E+14$ $3.76350 E+20$ $1.00006 E+01$ $3.6 H 2 G 2 E+29 \quad 1.00000 E+01$ $0.14400 E+24 \quad 1.00000 E+01$ B. $00704 E+29 \quad 1.00006 E+01$ $1.03081 E+30 \quad 1.00006 E+01$ $1.30135 E+30 \quad 1.00000 E+01$ $1.34992 E+30 \quad 1.00006 E+01$ $1.90520 E+30 \quad 1.000065+01$ $2.41248 E+30 \quad 1.00006 E+01$ $2.93410 t+30 \quad 1.00006 E+01$ $3.62849 E+30 \quad 1.00006 E+01$ $4.44492 E+30 \quad 1.00006 E+01$ $5.44037 E+30 \quad 1.00006 E+01$ $0.33236 t+30 \quad 1.00006 E+01$ $0.12571 t+30 \quad 1.00000 E+01$ $9.91363 E+30 \quad 1.00000 E+01$ $1.20193 E+31 \quad 1.00000 E+01$ $1.40968 E+31 \quad 1.00005 E+0$ 1. $10532 E+31 \quad 1.00003 E+01$ $2.16497 E+31 \quad 1.00005 E+0$ $2.32034 E+31 \quad 1.00003 E+01$ $3.16440 E+31$ 1.00005E+01 $3.81349 c+31 \quad 1.00003 E+01$ $4.50500 E+31 \quad 1.00005 E+01$ $3.497,1 E+31 \quad 1.00005 E+01$ $0.51288 E+31 \quad 1.00005 E+01$ $7.43327 t+31 \quad 1.00004 E+01$ $4.31060 E+31 \quad 1.00004 E+01$ $1.10270 E+32 \quad 1.00004 E+01$ $1.301+3 E+32 \quad 1.00004 E+01$ $1.53044 E+32 \quad 1.00004 E+01$ $1.7+302 E+32 \quad 1.00003 E+01$ $2.09261 E+32 \quad 1.00003 E+01$ $2.43264 E+32 \quad 1.00003 E+02$ $2.01079 E+32 \quad 1.00003 E+0$ $3.24043 E+32 \quad 1.00003 E+01$ 3.13111E+32 $1.00002 E+02$ $4.208242+32 \quad 1.00002 E+01$ C.06316E+32 $1.00002 E+01$ S.J1910E+32 $1.00002 E+01$ $0.25+20 E+32 \quad 1.00002 E+02$

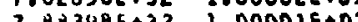

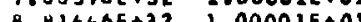
O.81446E+32 $1.00001 E+0$ l.830S5E $1.00001 E+0$ $1.2060 \% t+33$ l. $1.20609 t+33 \quad 1.00001 E+01$ 1.3 soant $1.0001 \mathrm{~s}$ $1.40261 t+33 \quad 1.00001 E+01$ $1.93911 E+33$, $\begin{array}{lll}1.32346 E+27 & 1.73911 E+33 & 1.00000 E+01 \\ 1.2635 E+21 & 1.07321+33 & 1.00000 E+01 \\ 1.20334 E+24 & 2.00402 E+13 & 1.00000 E+01\end{array}$ $\begin{array}{lll}1.32346 E+27 & 1.73911 E+33 & 1.00000 E+01 \\ 1.26035 E+2, & 1.07321 E+33 & 1.00000 E+01 \\ 1.20334 E+24 & 2.04402 E+33 & 1.00000 E+01\end{array}$ $305 \quad 1.40031 E-10-3.23444 E+01 \quad 1.00006 E+01$

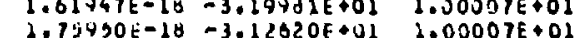
$1.90000-16-3.01547 E+01$ I.0007E+01

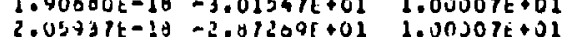
$2.21401 t=10-2.105404+01 \quad 1.00000+01$ $2.21691 \mathrm{E}-10-2.10340 \mathrm{k}+01 \quad 1.00000 \mathrm{0}+01$

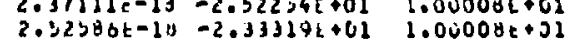


T0+396605.1 $18+376805 \cdot 8 \quad 36+35 T 6 \angle Q \cdot 4$ $10+30 \angle 6+9^{\circ} 1 \quad 18+799147 \cdot 8 \quad 26+392610^{\circ} 4$

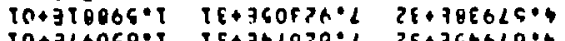

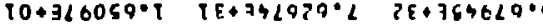
$10+792002 \cdot 1 \quad I E+7 E+T F E \cdot 2 \quad 2 E+305+\angle O \cdot 4$

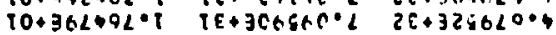
$10+36902 \beta^{\circ} T \quad I E+300 t 5 \% \cdot \theta \quad 2 \varepsilon+3056 \angle 9 \cdot 4$ $10+39616 \theta^{\circ} \mathrm{I}$ IE+3CO6CQ.0 $2 E+3546 \angle 5 \cdot 4$ $10+328090^{\circ}$ TO+3GEEEO $10+702001^{\circ}$ $10+360681 \cdot ?$ $10+326 \$<2 \cdot 8$

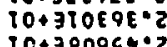
$10+380954 \cdot$ $10+392655^{\circ}$ $10+302950^{\circ} 5$ $10+36<69<\cdot 5$ $10+305920^{\circ} \cdot 2$ $10+316466^{\circ} 2$ $10+340617 \cdot \frac{1}{10+362642=8}$ $10+362642=8$ $10+36650 E^{\circ}$
$10+346626^{\circ}$ $10+3+5626^{\circ} E$
$10+3 E 6089^{\circ}$ $10+3 E E 089^{\circ} E$
$10+3480 E 0^{\circ}$ $10+348688^{\circ} E$
$10+386500 \cdot 4$ TO+ $10+300998-$ $10+722106.4$ $10+7182020$
$13+392606$ $13+992596.4$
$10+907912.6$ $10+301915.6$ $10+366094,5$ $10+76212 L^{\circ}$ $10+3 \angle E B O 0^{\circ} .5$ $10+321462 \cdot 9$

$10+304010^{\circ} 9$ $10+398676.9$ $10+346+1 E^{\circ} 2$ $10+30060 L \cdot 2$ $10+3465 E T \cdot 8$ $10+3650090^{\circ}$

$10+34160 T^{\circ} 6$

$10+3640<9=6$

$20+366620^{\circ}$

20+3IIEOT - I

$50+3162+E \cdot 1$

$20+306869 \cdot$ t

$20+34+924 \cdot 2$

$20+390105^{-} \varepsilon$

$20+38+E 8 L^{\circ}-4$
$20+3 \angle E 690^{\circ}$

$20+3 \angle E 59 \varepsilon^{\circ} ?$

$20+364852^{\circ}$,
$80+342650^{\circ}$

E0+301952 :

$60+369244^{\circ}$ ?
$60+306295^{\circ}$ ?

$60+306295^{\circ}$ 62+362054? तh

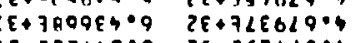
-1
-10 $16+385010.0$ IE+

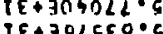
$1 E+35<516$.
$16+361<2$ TE+ $\rightarrow$ TOZGCE. $T E+T T A S h T^{\circ} 5$ TE+ $385 T 02 \cdot 6$ $1 E+7 E$ OCTT. IF + $7 E \$ \angle E 0^{\circ} 5$ $1 E+36010 b^{\circ}, 4$ IE + $39964 A^{\circ}$ TE+ JFOOBL $=0$ IE+ 7 TFFHL: IE+ TFEDOL*A $16+350020.4$ If $+7216+56=4$ $16+3+0 / 06=4$ $16+315246: 4$
$18+700076 \cdot 4$ $16+79560 \%+4$ TE+ 3PCTPSD $1 E+75+C 04 \cdot 9$ II $+3 \angle P F C=4$ $18+3 \$ 512404$ $1 E+7 \rightarrow 4 L T+\cdots$

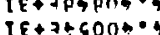
IF+705E6F. TE+30TLPE. $T E+71$ LQLE. $\tau E+3 \angle+21 E \cdot$ TE+ $T E+7 z 2 \angle द E \cdot 4$

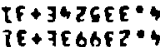
IE+36E\$Zn० TE+3STTEO०E IE+ $315520^{\circ} E$ $16+34,602,5$

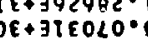
$09960 \cdot 9 \cdots ?$ $41+398999^{\circ} 2 \quad 10-70<400^{\circ} z \quad F 0+700.298 \cdot 1$

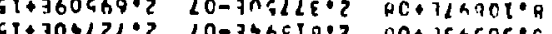

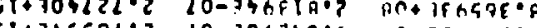

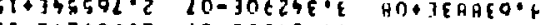

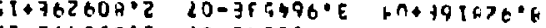

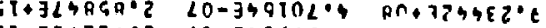

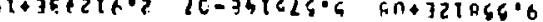

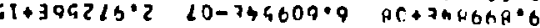

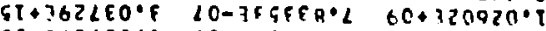

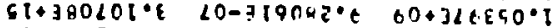

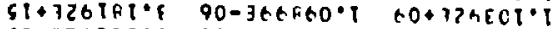

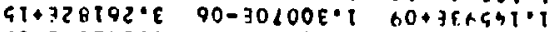

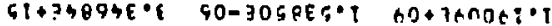

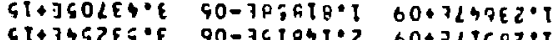

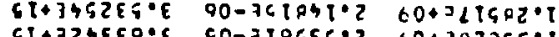

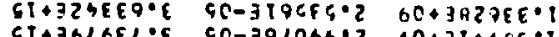
$S T+36 L E E L \cdot F$

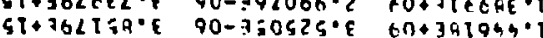
$51+75096.6$ $51+387 \varepsilon 677^{\circ}$ $5 T+75 A 222 \cdot 4$ $5 T+38985 E=4$
$S T+3 \rightarrow R 00 S \cdot 4$ $5 I+38 \rightarrow+490$ $51+38<400^{\circ} 4$
$51+34+970^{\circ}$ $51+34+976^{\circ} 4$
$41+3819 I^{\circ}$ $5 T+3$ TRET $5 I+39121 E^{\circ}$

$51+726964^{\circ} \cdot 4$
$51+341669^{\circ}$

$51+324688^{\circ}$

$51+360067.9$ $4 T+3809450$ $51+3115920^{\circ}$
$51+32+550$.

$5 T+76 R L$ ?

$5 T+31+E \angle 5^{\circ}=2$

$51+792691$.

$S T+702687^{\circ} \mathrm{P}$
$S T+102429^{\circ}$

$6 T+30242 \theta^{\circ}$
$6 T+72541 T^{\circ}$

$5 T+325 \angle T=\circ$
$4 T+721000^{\circ}$

$5 T+32100 \pi^{\circ} 6$

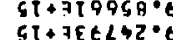

$5 T+3 E E \angle \$ 2 \cdot 6$
$5 T+36 \$ 29 T^{\circ}$

$S t+399 E 0 L=E$

$51+3 I R I L B=1$
$6 I+3 L O C 5 S^{\circ}$

$51+3 \rightarrow \angle S H+01$

ST+गTTTLE'T
ST+ $T$ STTEण

$51+322092$

$5 t+366002 \cdot 1$

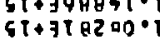
$0 \quad s t+7 t E \angle 6 T$ ONJ 0-350525.8 6n+3A194.'?

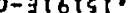

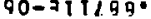

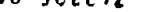

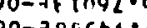
$80-306456$. $90-304260^{\circ}$
$40-301 \angle 60^{\circ}$ Cी- $302680^{\circ}$ SO-30EदIs: GCI-726TFL co- 351004 ? c0-3heOIs. SO- 36 EQEH $50-350890^{\circ}$

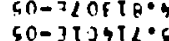

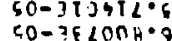
$4 \cap-3 \varepsilon r_{1}<I T^{\circ}$ CO-34512 40-309A9 $30-786014+1$
$+5-31081204$

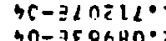
$\rightarrow 0-3<<095^{\circ}$

$90-j \angle E L A T \cdot E$ $40-3025100^{\circ}$
$40-7<5205$. \$0-35906 CO-36<LLI: $60-70 E \angle 160^{\circ} ?$
$20-3601 \angle 0^{\circ}$ $20-3501 \angle 0^{\circ}$

To- $746000^{\circ}$

TC- $T$ T LES4:
nO+ JE TEZ4:

$n 0+765900^{\circ}$
$10+32197+0^{\circ}$

$10+3 \angle E A L E \cdot 4$

$20+711146 \cdot 1$
$20+760890 \cdot 2$ $20+760890 \cdot 2$
$20+36 F 600 \%$ $60+7 n z<0<.5$
$60+31<c$

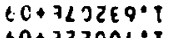
tod 052002 . T

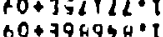
$+0+312526^{\circ} 1$ $60+1118000^{\circ} 2$ $10+76+5+0.2$ c0+710/91? EC+7004p2. $60+7027 F E \cdot ?$ $60+1 F^{\prime} F 09 \cdot 2$ $60+1 \angle \mathrm{FOZL}^{\circ} \mathrm{Z}$

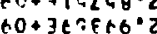

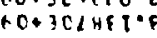
An $3945+2 \cdot E$ 1.0+311065+.

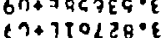

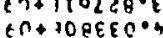
$c 0+3\{2<52 \cdot n$ $60+3450.7504$ $6 n+791090=5$ $60+314600^{\circ} 5$

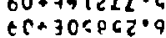
$60+\exists 16920^{\circ} \mathrm{L}$

$60+755189^{\circ}$

$0 \tau+39956 z^{\circ}$

$01+796158^{\circ} 1$

CT+ THLOEE. OT+FIET2C. $0 T+346 \angle E H^{\circ} 5$
$0 T+700 E \angle 5 \circ 9$ $0 T+760 E<5 \cdot 0$ $07+726+4 y^{\circ} \cdot 2$

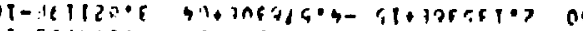
$a t-7630,060$

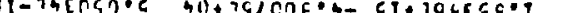

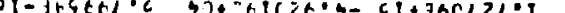

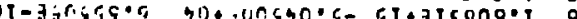

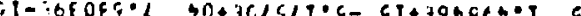

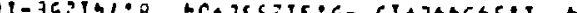
व-0ionn.

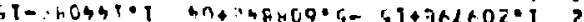

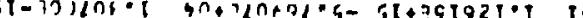

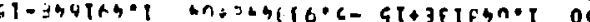

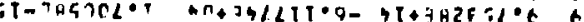

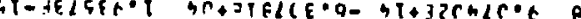

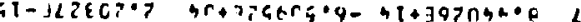

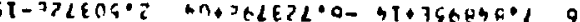

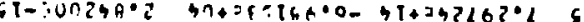

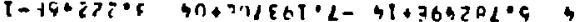

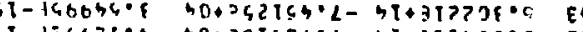

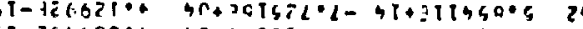

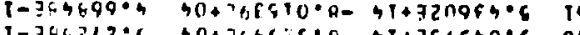

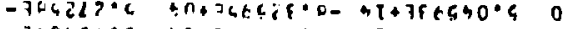

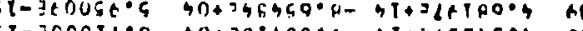

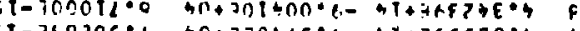

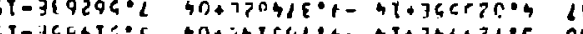

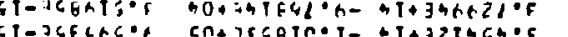

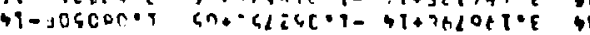

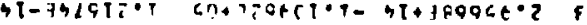

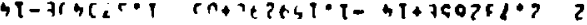

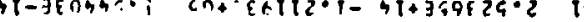

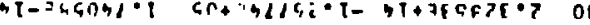

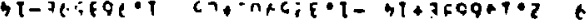

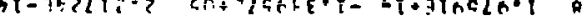

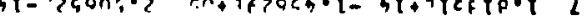

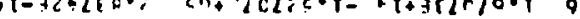

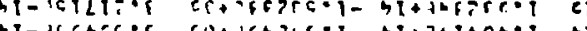

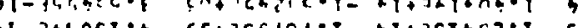

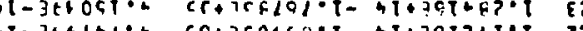

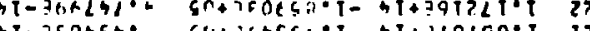

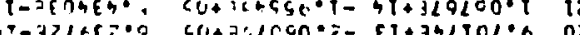

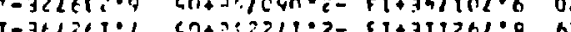

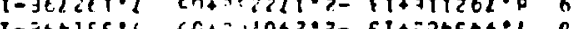

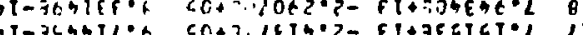

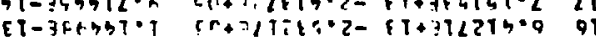
EI-J4 EI-35014L.

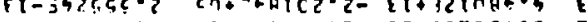

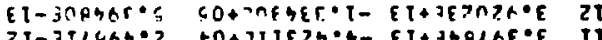

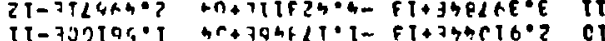

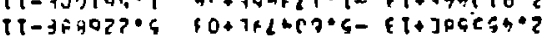
Ot-joOCha.t

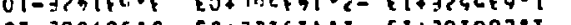
bo-3gtces 60-3ctssi

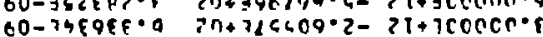
$0-30012+1$ $3 n+716409 \cdot 2-21+1000 c 0 \cdot 1$

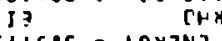

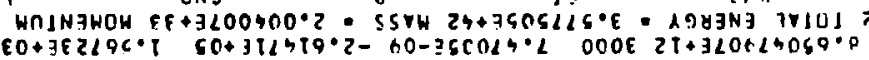

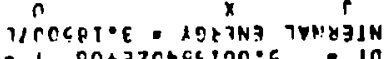
i ootzonagino.a 


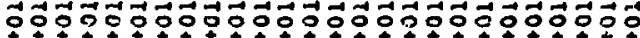

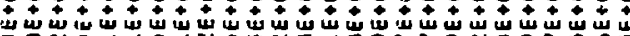

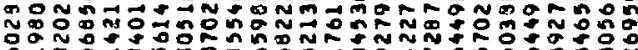

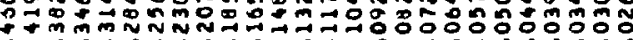

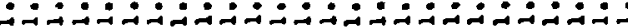

דี

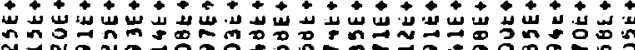

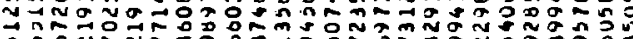

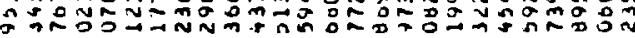

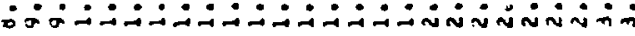

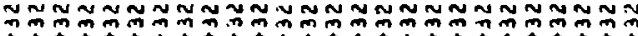

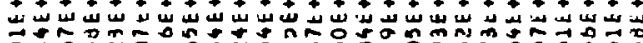

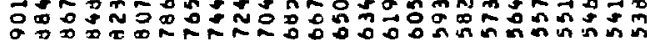

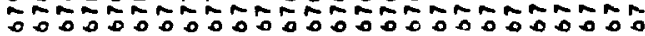

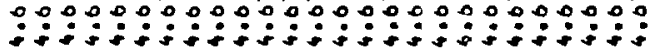

nמngn

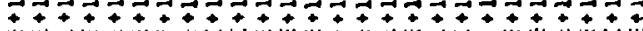

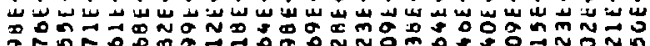

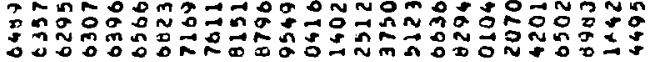

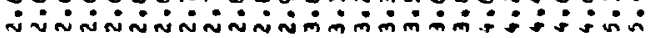

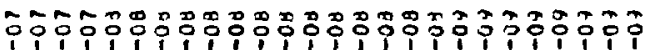

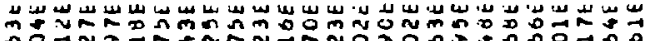

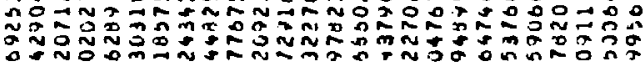

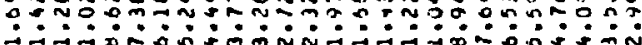

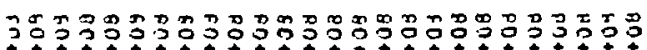

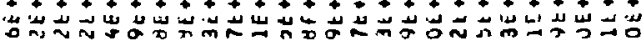

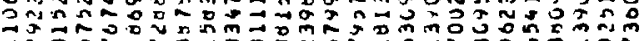

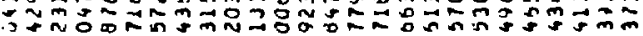

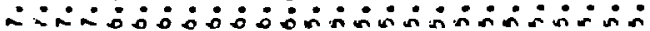

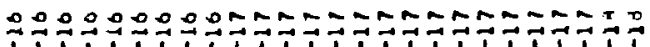

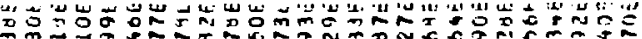

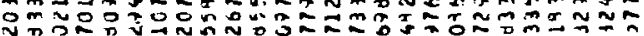

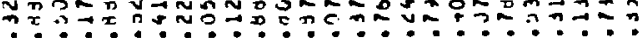
miñ

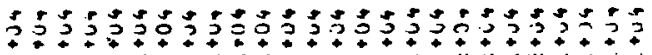

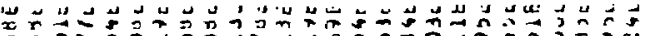

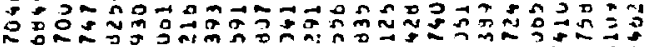

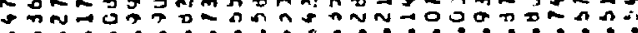

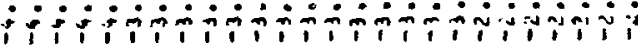

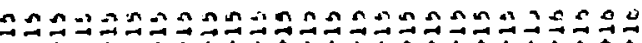

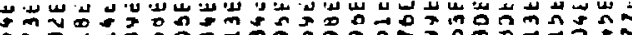

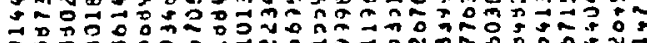

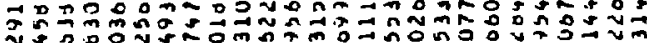

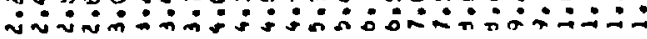

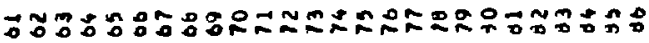

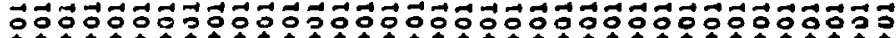

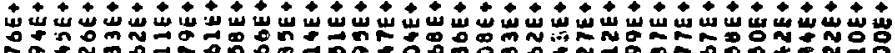

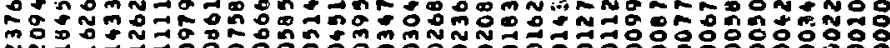

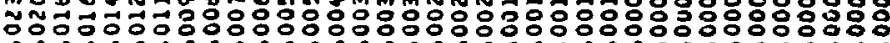

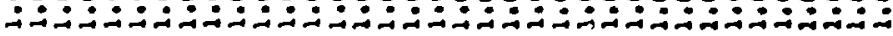

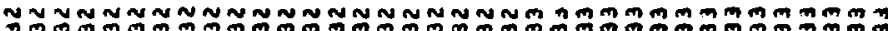

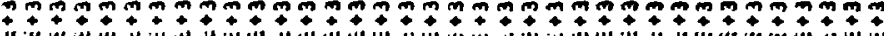

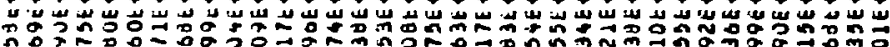

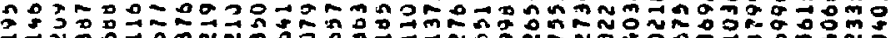

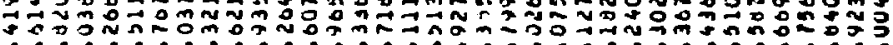

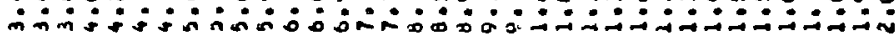

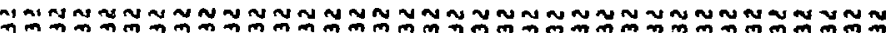

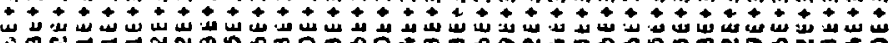

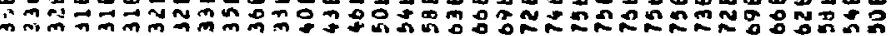

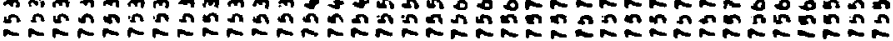

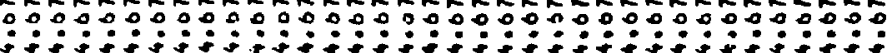

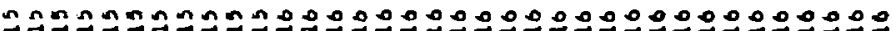

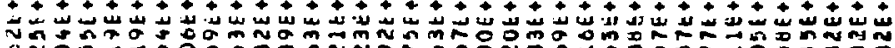

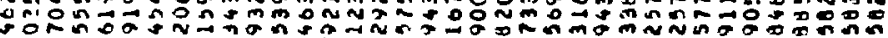

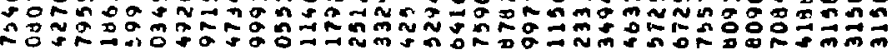

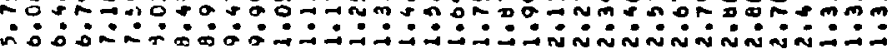

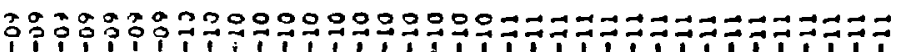

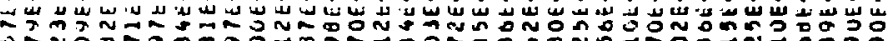

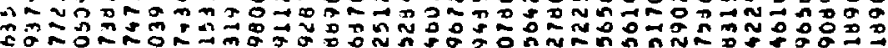

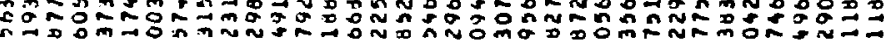

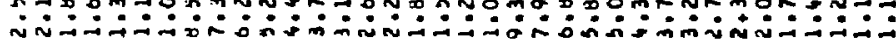

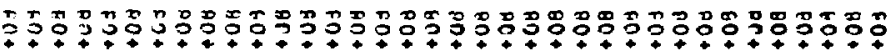

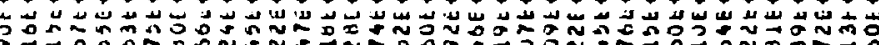

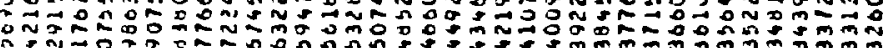

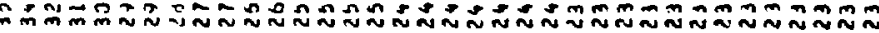
An

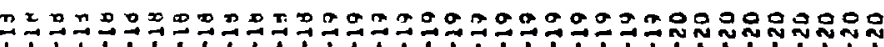

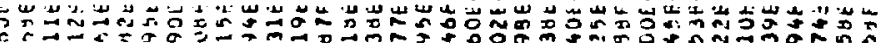

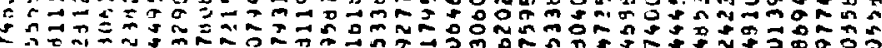

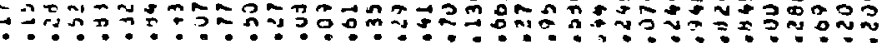

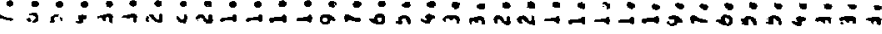

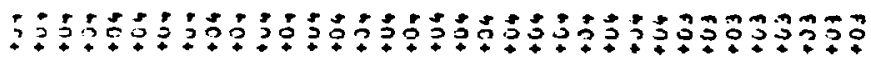

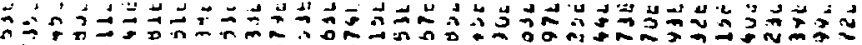

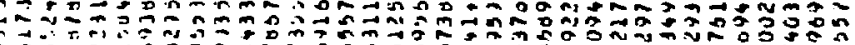

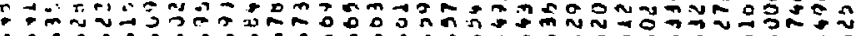

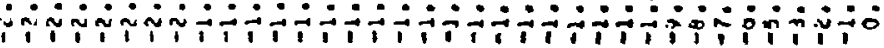

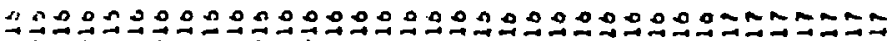

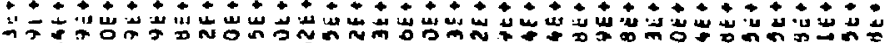

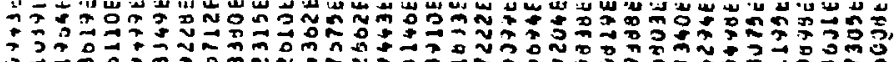

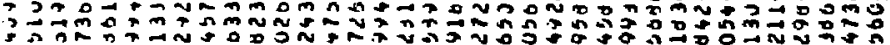

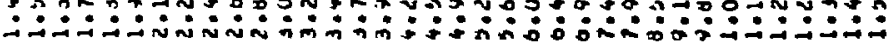


APPENDIX B 
*DELETE SV. 23 LATA LFILM,KPR,NM/10,1,20/

*DELETE SV.313 (J),J=1,2001/200*3.02232E-091

DATA (REV ( J), J

*DELETE SV. $370,5 V \cdot 37$

$\operatorname{RHO}(J)=2.4 E-0$,

$P(J)=1.79543 E+03$

IF (J.LE. 5) $P(J)=5.37152 E+14$

*DELETE SV.S13,SV.624

CND $(J)=0.5 *(\operatorname{MUVISC}(J-L)+\operatorname{MUVISC}(J)) / P R$

* DELETE SV.0II

DATA PR/100.1

HELETE SV.616,SV.617

MUVISC $(J)=2.4 E+07$

*DELETE SV.527

*DELETE SV.78

* INSERT SV.jog

IF (IUMAX GT, -1) RETURN

+ INSERT SV. 26

$G=0$.

*DELETE SV. 22

OATA POVCEN/O.S1I

* INSERT SV. 26

CALL UNJROP 


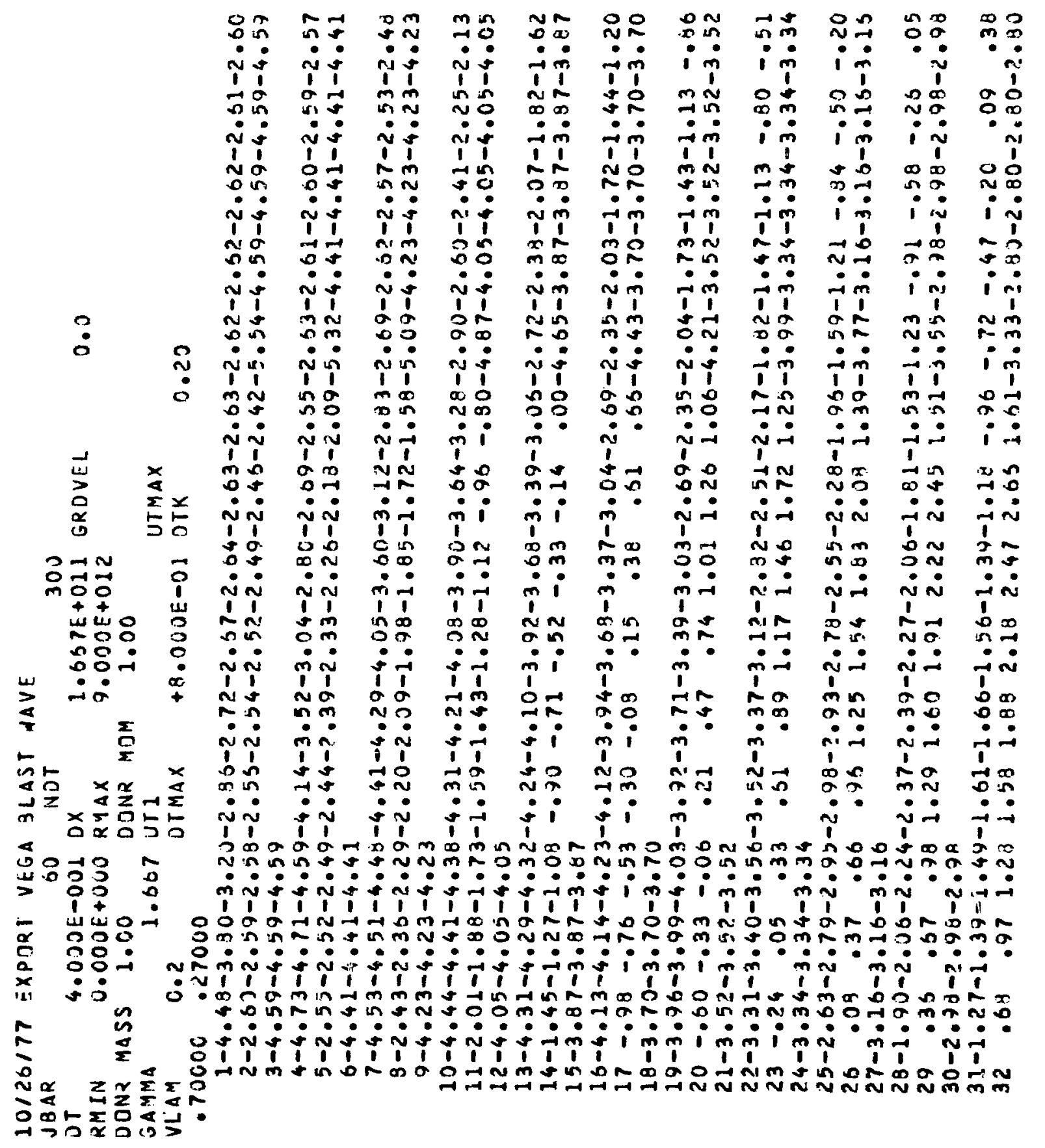

HNm $\operatorname{tin}$ D

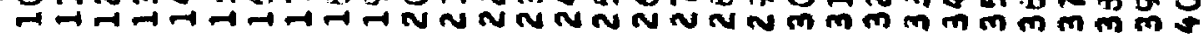




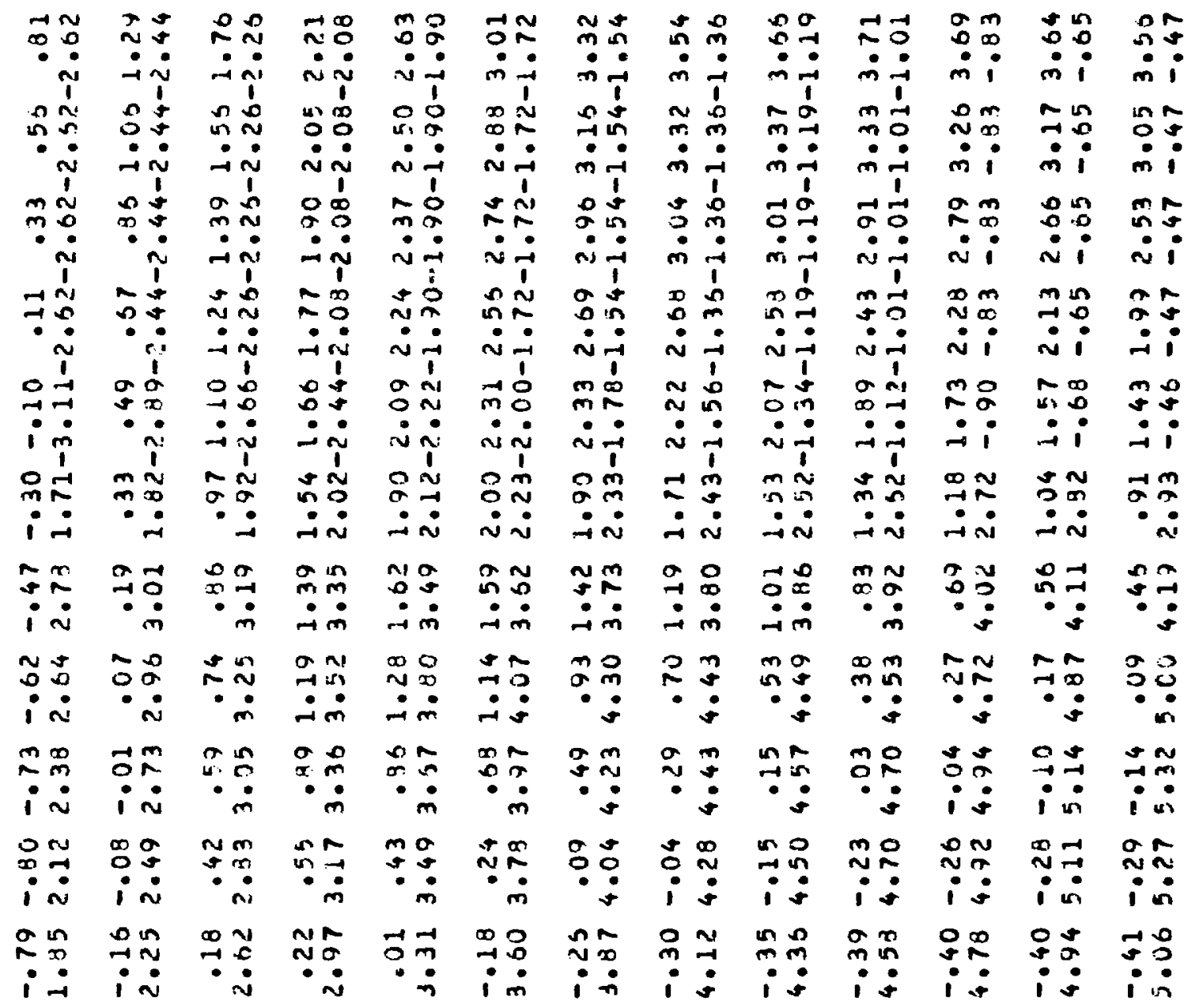

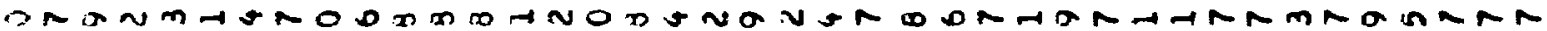

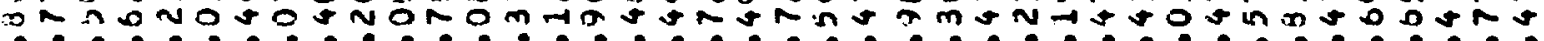

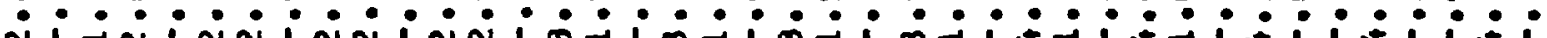
N IN Jan

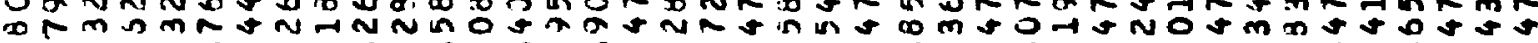

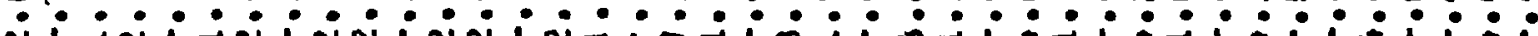
i 1 -

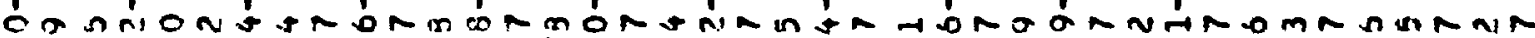

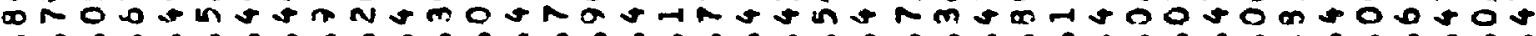

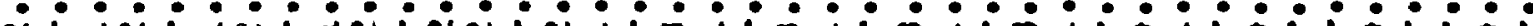
N1 $\rightarrow$ I I

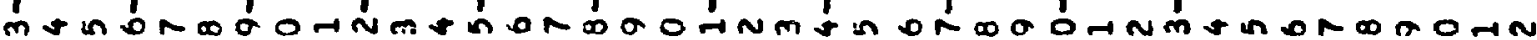

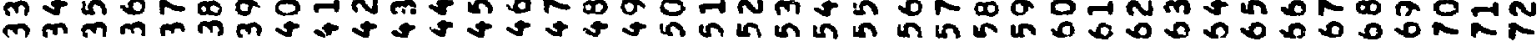




\begin{tabular}{|c|c|c|c|c|c|c|c|c|c|c|c|c|}
\hline $\begin{array}{ll}0 & a \\
+ & n \\
m & 1\end{array}$ & $\begin{array}{l}m=1 \\
m=1 \\
\dot{m}:\end{array}$ & $\begin{array}{l}n \\
\\
\dot{m}\end{array}$ & $\stackrel{0}{a} \stackrel{n}{m}$ & 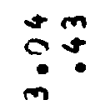 & $\begin{array}{l}\vec{n} \\
\therefore \\
\therefore !\end{array}$ & $\begin{array}{l}0 \\
\stackrel{R}{R} \\
\ddot{n}\end{array}$ & $\begin{array}{l}\vec{u} \tilde{r} \\
\dot{\mathrm{s}}\end{array}$ & 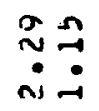 & $\vec{n} \tilde{n}$ & $\begin{array}{l}\dot{\infty} \tilde{N} \\
\dot{0}:\end{array}$ & $\overrightarrow{0}:$ & \\
\hline 弯 & 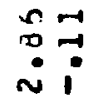 & $\tilde{\sim} \tilde{r}$ & \begin{tabular}{l}
$u N$ \\
\hdashline \\
$\sim$
\end{tabular} & in & & $\begin{array}{l}\sim a \\
\sim \\
\sim\end{array}$ & & & $\begin{array}{l}0 N \\
\because n \\
\because n\end{array}$ & & $\tilde{a}$ & \\
\hline $\begin{array}{l}N \\
\pm \\
\sim\end{array}$ & $\begin{array}{l}m \vec{l} \\
\dot{n} \\
\dot{v}\end{array}$ & $\begin{array}{l}\sim \\
\sim\end{array}$ & $\underset{n}{*} \cdot \stackrel{n}{*}$ & $\begin{array}{l}\tilde{y} \\
0 \\
0\end{array}$ & $\begin{array}{l}\sim 0 \\
\ddot{0} \\
-1\end{array}$ & $\begin{array}{l}\because 9 \\
\because \approx\end{array}$ & $\underset{m}{a}$ & $\stackrel{\infty}{\infty}$ थn & in & ? & $\because \stackrel{n}{\because}$ & $\begin{array}{l}N \tilde{N} \\
\dddot{N} \\
i \sim\end{array}$ \\
\hline $\begin{array}{ll}\infty & 0 \\
\infty & 1 \\
-1 & 0 \\
-1 & 1\end{array}$ & $\begin{array}{l}\ddot{0} \\
\because \ddot{1} \\
-1\end{array}$ & $\because \tilde{O}$ & $\stackrel{3}{0} \stackrel{n}{\sim}$ & \begin{tabular}{l}
$n=$ \\
$n$ \\
\hdashline
\end{tabular} & $\ddot{m}:$ & $\stackrel{2}{\rightarrow} \underset{0}{\longrightarrow}$ & & & $\because n$ & ? & $\begin{array}{l}m \Omega \\
i \approx\end{array}$ & \\
\hline $\begin{array}{l}m \\
n \\
n \\
n\end{array}$ & $\begin{array}{l}\because \\
\because i \\
\therefore:\end{array}$ & $\stackrel{0}{\rightarrow} \vec{u}$ & $\stackrel{9}{9}$ & $\begin{array}{l}0 \\
0 \\
0 \\
\vdots\end{array}$ & $\overrightarrow{0}$ & \begin{tabular}{l}
0 \\
0 \\
\hdashline
\end{tabular} & $\ddot{m} \vec{m}$ & $\because$ in & $\underset{r}{\sim} \underset{r}{\sim}$ & & & \\
\hline $\begin{array}{l}n \\
0 \\
\dot{m}\end{array}$ & $\stackrel{n}{2} \ddot{n}$ & $\begin{array}{l}m \\
0 \\
0\end{array}$ & On & in $\overrightarrow{5}$ & $\stackrel{n}{m} \cdot \vec{n}$ & $\because \stackrel{0}{\because}$ & $\begin{array}{l}10 \\
0 \\
0\end{array}$ & & $\begin{array}{l}\sim \tilde{n} \\
\because \ddot{n} \\
\dot{m}\end{array}$ & $\begin{array}{l}m \vec{r} \\
i \dot{m}\end{array}$ & & \\
\hline$\stackrel{m}{n}$ & $\dddot{n} m$ & $\stackrel{0}{\dddot{N}}$ & $\stackrel{2}{\sim}$ & $\because$ & $\overrightarrow{0}:$ & $\begin{array}{l}\rightarrow 0 \\
\Rightarrow ? \\
\because ?\end{array}$ & $\stackrel{v}{\sim}$ & & $n$ & 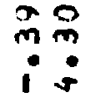 & & \\
\hline $\begin{array}{l}0 \\
0 \\
0 \\
0 \\
0\end{array}$ & $\begin{array}{ll}1 & 9 \\
0 & 3 \\
1 & \text { in }\end{array}$ & $\begin{array}{l}5 \tilde{0} \\
1:\end{array}$ & \begin{tabular}{l}
23 \\
\hdashline$: 3$ \\
10
\end{tabular} & \begin{tabular}{l}
0 \\
\hdashline$: 0$ \\
\hdashline$: 0$
\end{tabular} & $\begin{array}{l}m \vec{n} \\
\because \vec{s}\end{array}$ & $\stackrel{9}{m}$ & $\begin{array}{l}n \\
m \\
1\end{array}$ & & $\begin{array}{l} \pm \approx \\
\vdots \approx \\
1\end{array}$ & & & $\begin{array}{l}\tilde{s} \\
\mathrm{~s}\end{array}$ \\
\hline $\begin{array}{l}\infty \\
\overrightarrow{5} \\
i \\
1\end{array}$ & $\begin{array}{l}m \dot{n} \\
\because \dot{m}\end{array}$ & $\begin{array}{l}n \\
\sim \\
\sim\end{array}$ & $\tilde{N} N$ & 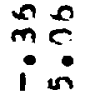 & 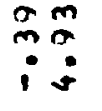 & $\begin{array}{l}10 \\
40 \\
\because:\end{array}$ & $\begin{array}{l}+\infty \\
0 \\
1\end{array}$ & $?$ & $\begin{array}{l}13 \\
18 \\
18\end{array}$ & & 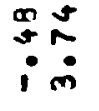 & $\begin{array}{l}33 \\
13\end{array}$ \\
\hline 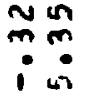 & $\ddot{m}$ & $\stackrel{0}{m}$ & $\begin{array}{l}50 \\
: \cong \\
10\end{array}$ & $\begin{array}{l}\because 50 \\
\because \because 8\end{array}$ & 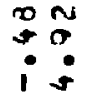 & & s: & & $\begin{array}{l}n=n \\
i= \\
i=\end{array}$ & & जu & \\
\hline$\stackrel{\square}{\because}$ & $\because 0$ & \& in & $\because \approx$ & $\stackrel{n}{n}$ & 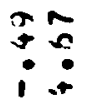 & $\because ?$ & $\begin{array}{l}0 \\
? \\
1\end{array}$ & $\stackrel{5}{\square} \stackrel{0}{\square}$ & F? & & $\because \vec{N}$ & $\because$ \\
\hline
\end{tabular}

ToGNOMNORN

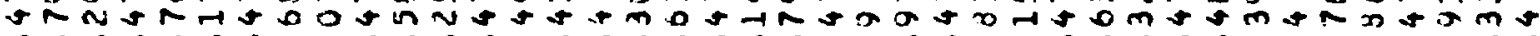

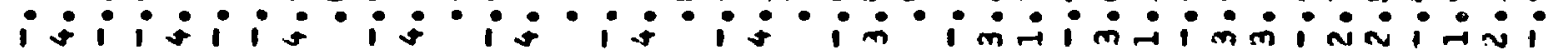

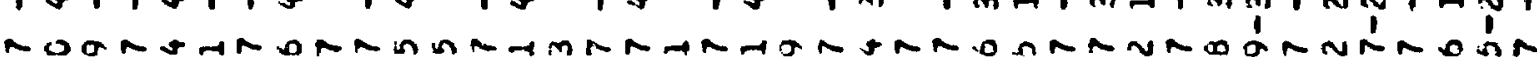
I TN

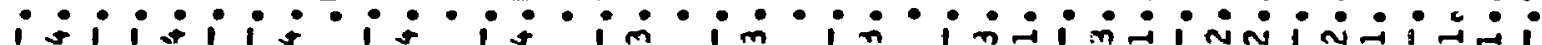
n F。

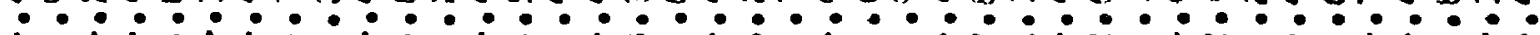

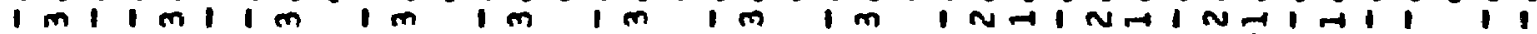
$m$ - In 0 -

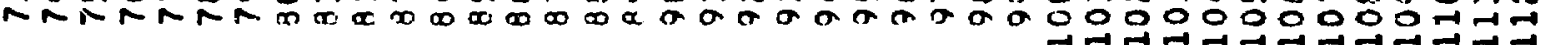




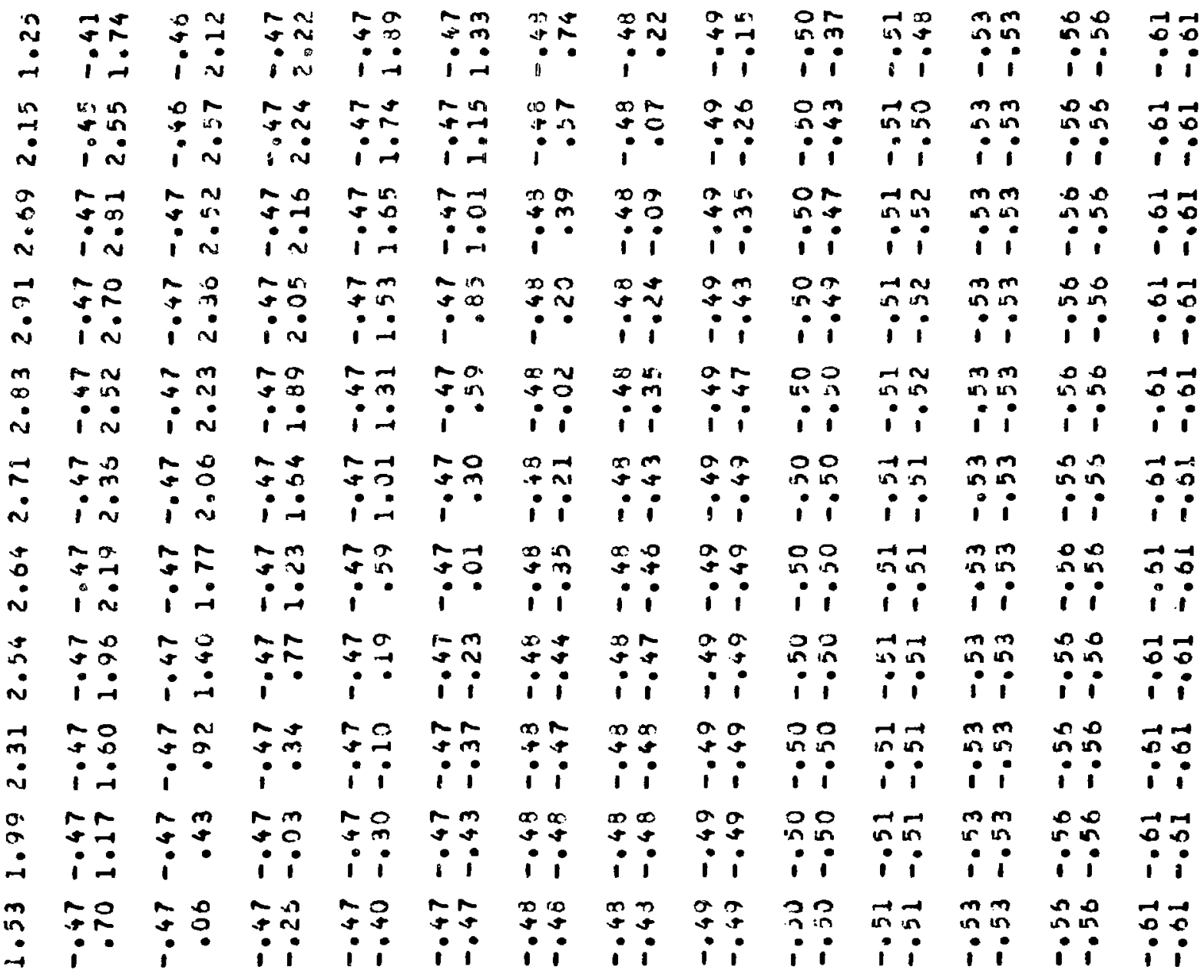

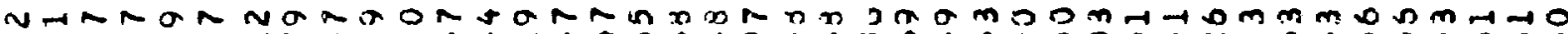

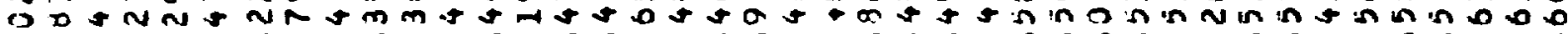
自:

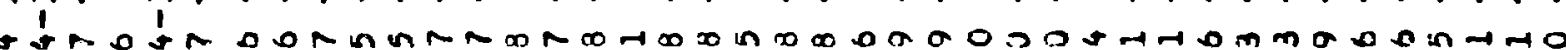

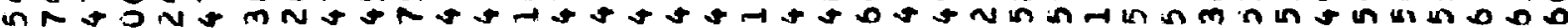

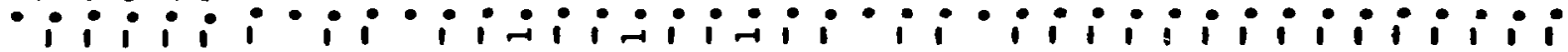

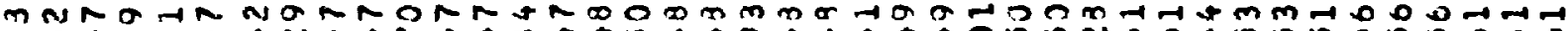

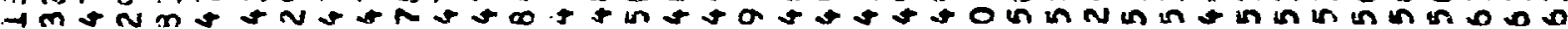

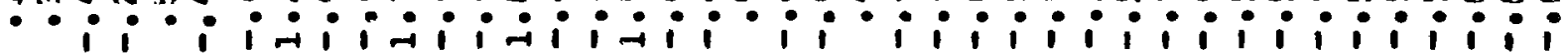
$m \sim$ u

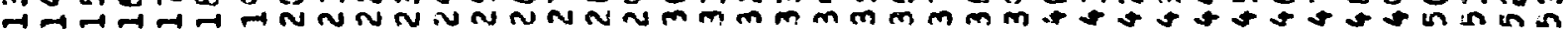

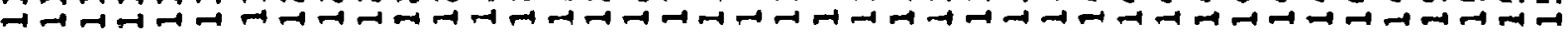

$\rightarrow N m$ t N N N $N$ N $N$ N 
APPENDIX 5 
1 *IDENT PRDB

2 *DELETE SV.23.

3

4

*DELETE SV.313

DATA LFILM,KPR,NM $150,5,200 /$

6 *DELETE SV.25\%

$7 \quad U(J P \geq)=U(J P I)+D E L U$

S $\quad U(J P 3)=U(J P 2)+D E L U$

$9 \quad R H O(1)=3.674 E-21$

$10 \quad E I(J P Z)=2$.*EI(JPI)-EI (JBAR)

11 *DELETE SV.201

12 EI (1) $=2.65 E+14$

13 RDELEJE SV.370,SV.373

$14 \quad R H O(J)=3.674 E-21$

$15 \quad E I(J)=2.65 E+14$

$16 \quad P(J)=($ GAMMA-1.)*R+O(J)*EI(J)

17 *DELETE SV.375

13 *OELETE SV.613,5V.624

$27 \quad C N D(J)=0.5 *(M U V I S C(J)+M U V I S C(J-1)) / P R$

20 *DELETE SV.130

$21 \quad H A(K+1, L-5)=-1$.

22 \#DELETE SV.78

23 *DELETE SV.527

24 *DELFTE SV.2?B

25 *DELETE $\$ V .374$

26 U(J) $\triangle U T 1$

27 *DELETE SV.87

$23 \quad S U M=1.33 E+26$

29 *DELETE SV.9J

$30 \quad D P H I(J P \geq)=S U M / X(J P \geq) * \# 2$

31 *OELETE SV.204

32 RHOL (1)=3.574E-21

$33 \quad t I(1)=2.65 E+14$

$34 \quad U T(J P 2)=U T(J P L)+3 E L U$

$35 \quad U T(J P 3)=U T(J P 2)+D E L U$

$36 \quad E I(J P 2)=2$.*EI (JPI)-EI(JQAR)

37 DELETE SV.552

39 DO $10 \mathrm{~J}=2, \mathrm{JP3}$

39 * LNSERT SVIZOS

40 SUBRJUTINE ASJLN(X,P,EI,RHT,U,GAMMA,JPI) 


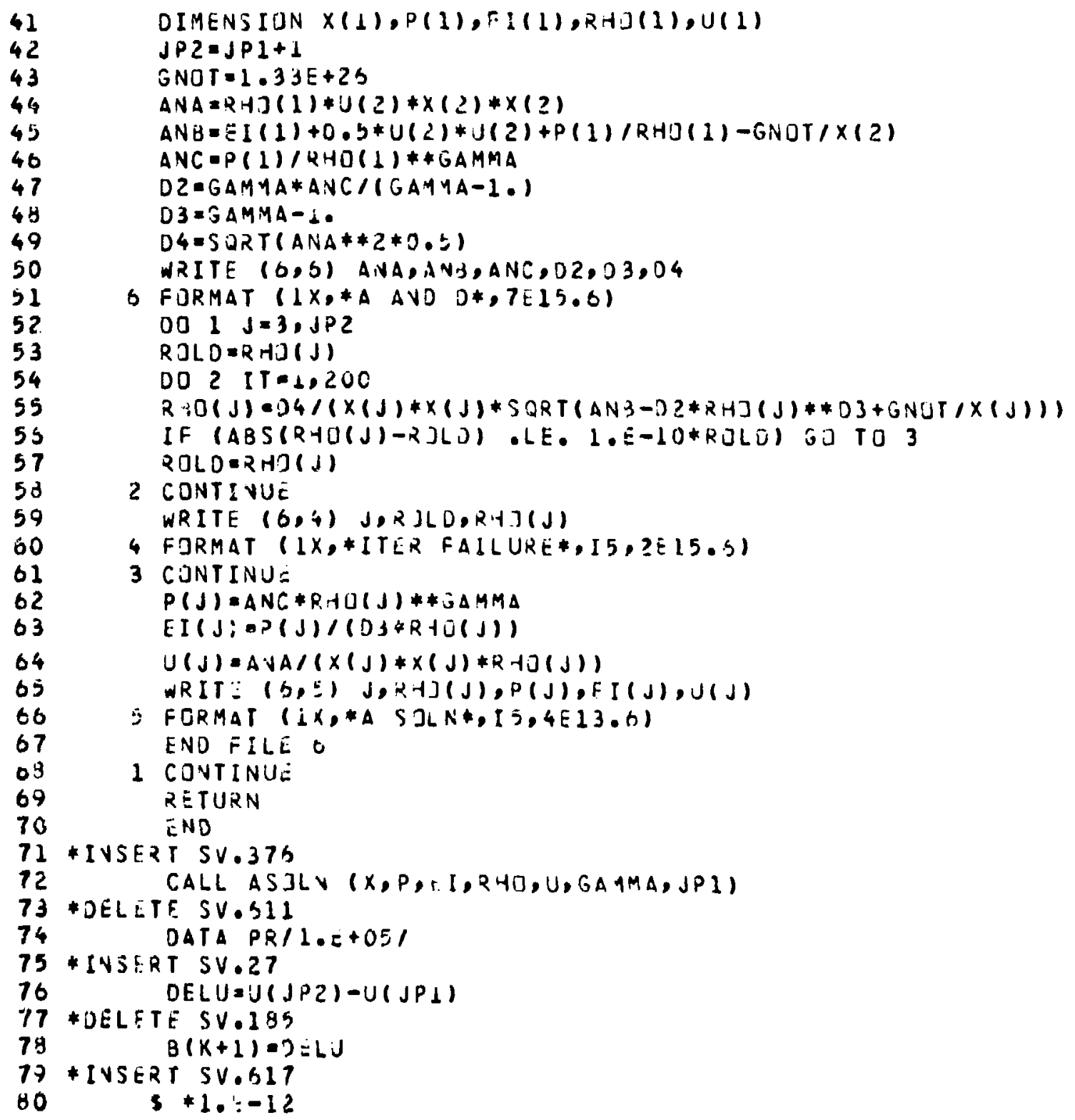




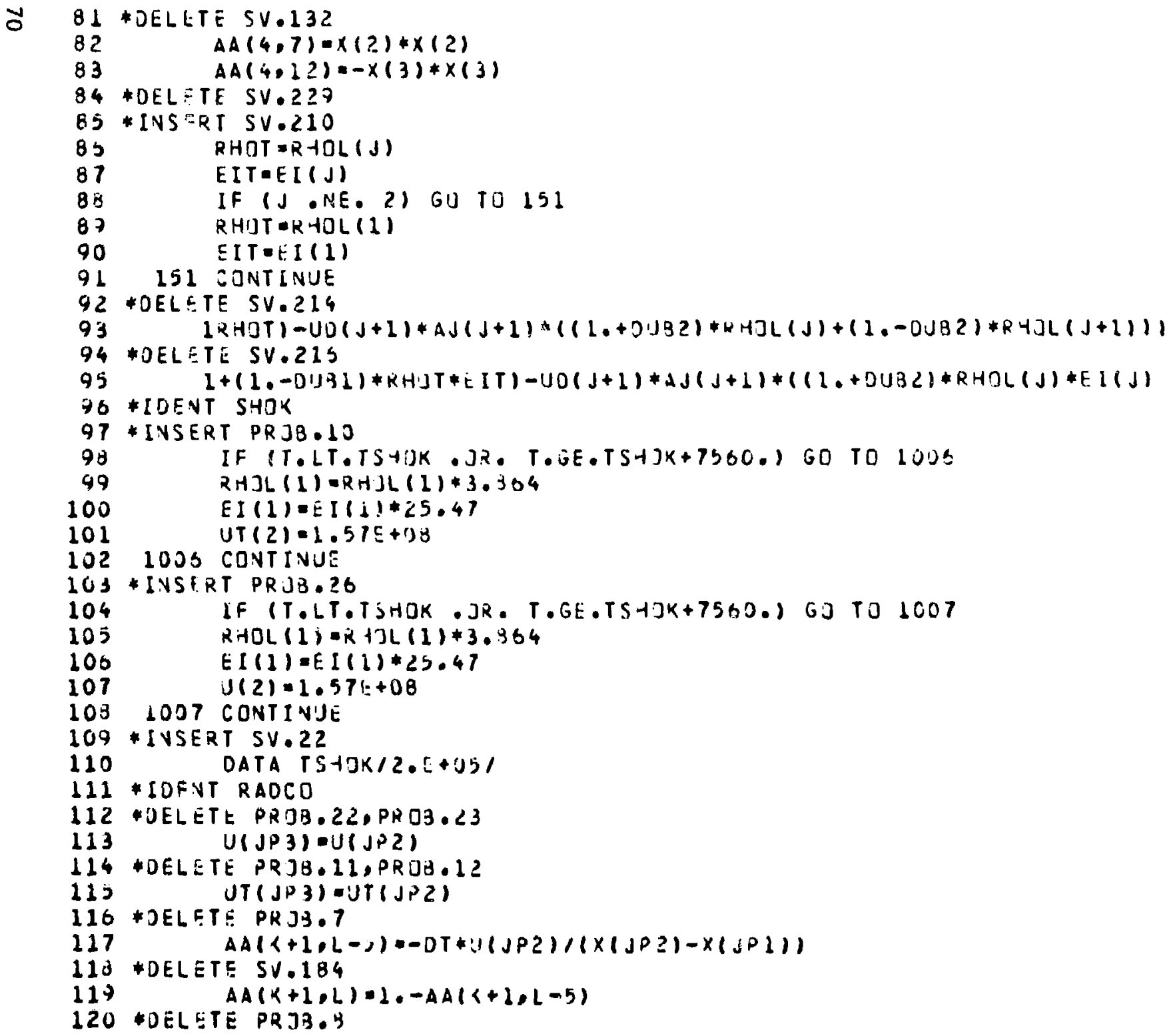




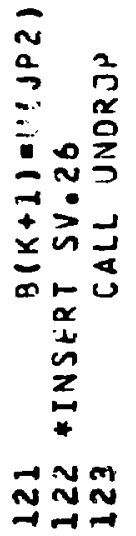


10/26/77 EXPORT VEGA SOLAR WINO

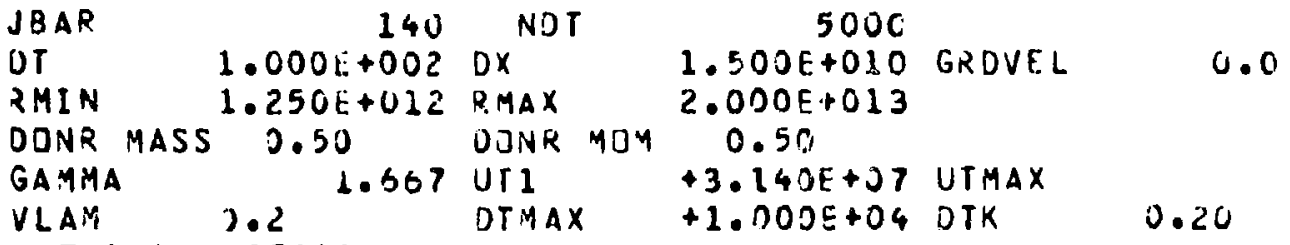

$.70000 \quad .27000$

$1-4.48-3.80-3.20-2.85-2.72-2.67-2.64-2.63-2.63-2.52-2.62-2.62-2.61-2.60$

$2-2.50-2.59-2.53-2.56-2.54-2.52-2.49-2.46-2.42-5.54-4.57-4.59-4.39-4.59$ $3-4.59-4.57-4.59$

$4-4.73-4.71-4.59-4.14-3.52-3.04-2.80-2.09-2.65-2.63-2.61-2.60-2.59-2.57$ $5-2.55-2.52-2.49-2.44-2.39-2 \cdot 33-2 \cdot 26-2 \cdot 18-2.09-5 \cdot 32-4.41-4 \cdot 41-4 \cdot 41-4 \cdot 41$ $6-4 \cdot 41-4,41-4,41$

$7-4.53-4.51-4.43-4.41-4.29-4.05-3.60-3.12-2.83-2.69-2 \cdot 62-2 \cdot 57-2.43-2 \cdot 43$ $8-2.43-2.36-2.29-2.20-2.09-1.78-1.85-1.72-1.58-5.09-4 \cdot 23-4 \cdot 23-4 \cdot 23-4.23$ $7-4 \cdot 2.3-4 \cdot 23-4 \cdot 23$

$10-4 \cdot 44-4.41-4.38-4.31-4.21-4.08-3 \cdot 7 j-3.34-3.28-2 \cdot 7 j-2 \cdot 3 j-2 \cdot 41-2 \cdot 25-2 \cdot 13$ $11-2.01-1.88-1.73-1.59-1.43-1.29-1.12-.90-.5 j-4.8754 .05-4.05-4.05-4.05$ $12-4.05-4.05-4.05$

$13-4.31-4.29-4.32-4.24-4 \cdot 10-3.92-3.69-3.39-3.06-2.72-2.38-2.07-1.92-1.62$ $14-1.45-1.27-1.03-.90-.71-.92-.33-.14 \quad .00-4.65-3.87-3.87-3.87-3.87$ $15-3.87-3.87-3.87$

$16-4.13-4.14-4.23-4 \cdot 12-3.94-3.58-3.37-3.04-2.69-2 \cdot 35-2 \cdot 03-1 \cdot 72-1 \cdot 44-1 \cdot 20$ $17-.98-.76-.53-.30-.08 \quad .15 .34 \quad .31 \quad .66-4.43-3.70-3.70-3.70-3.70$ $18-3 \cdot 7 \mathrm{C}-3.7 \mathrm{~V}-3.70$

$19-3.96-3.97-4.03-3.72-3.71-3.39-3.03-2.59-2.35-2.04-1.73-1.43-1.13-.86$ $20-.30-.33-.06 \quad .21 .47 .741 .011 .261 .06-4.21-3.52-3.52-3.52-3.52$ $21-3.52-3.52-3.52$

$2.2-3.31-3.40-3.55-3.52-3.37-3.1 ?-2.82-2.51-2.17-1.32-1.47-1.13-.80-.31$ $23-.24 \quad .05 .33 .61 .891 .1710461 .721 .25-3.99-3.34-3.34-3.34-3.34$ $24-3.34-3.34-3.34$

$25-2.63-2.79-2.95-2.98-2.93-2.78-2.55-2.25-1.96-1.59-1.21-.84-.50-.20$ $26.03 .37 .66 .981 .251 .541 .832 .041 .39-3.77-3.10-3.16-3.16-3.16$ $27-3 \cdot 16-3.16-3.16$

$28-1.90-2.06-2.24-2.37-2.39-2.27-2.06-1.81-1.53-1.23-.71-.58-.26 \quad .05$ $29.35 \quad .37 \quad .981 .291 .60 \quad 1.912 .22 \quad 2.45 \quad 1.51-3.55-2.98-2.98-2.98-2.98$ $30-2.73-2.98-2.98$

$31-1.27-1.39-1.49-1.61-1.66-1.56-1.39-1.18-.96-.72-.47-.20 \quad .09 \quad .38$ $32.63 .971 .281 .581 .892 .182 .472 .651 .61-3.33-2.80-2.80-2.80-2.80$ 


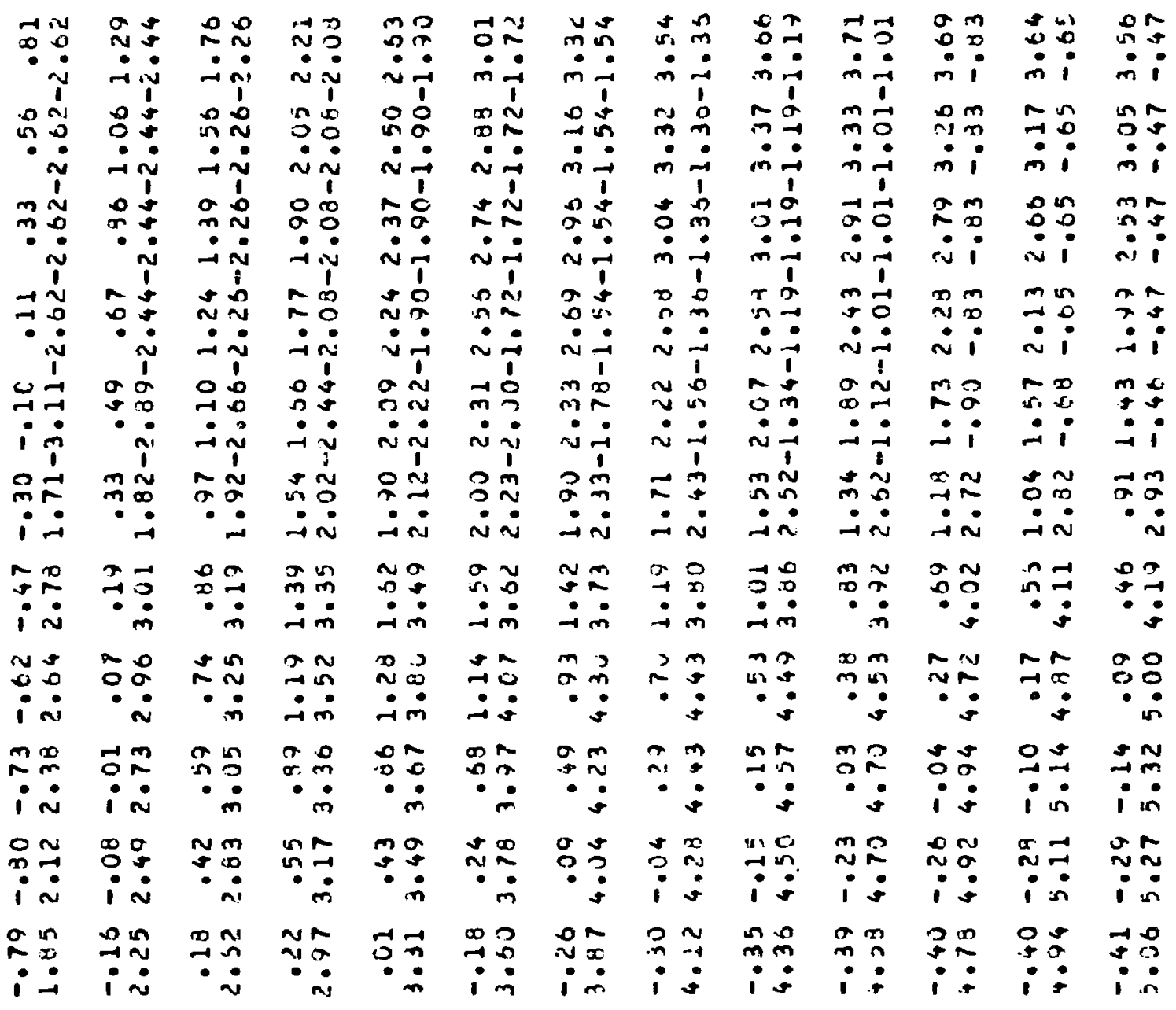

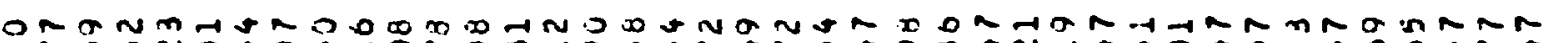

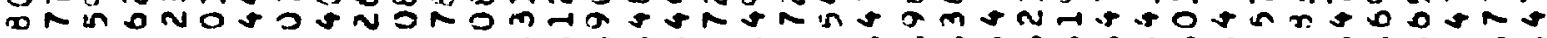

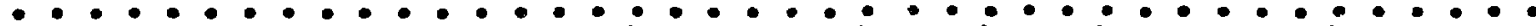
N 1 Tom

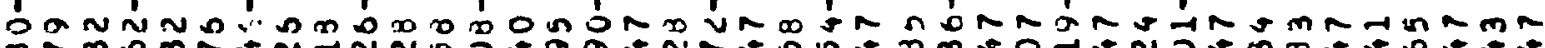

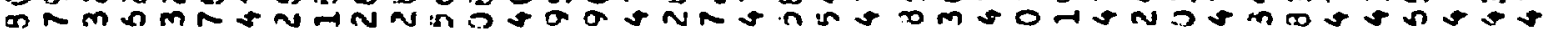

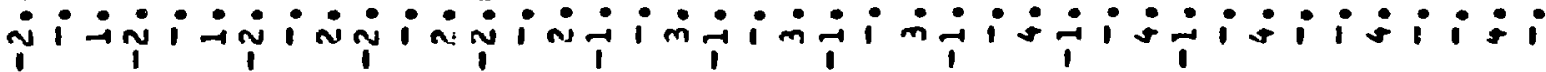
br

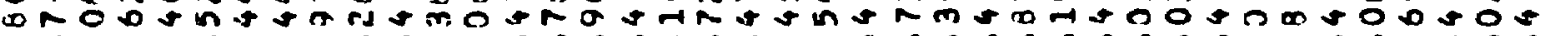

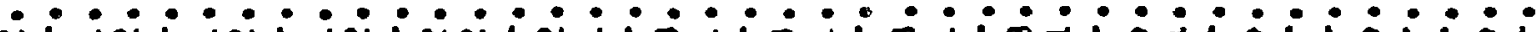

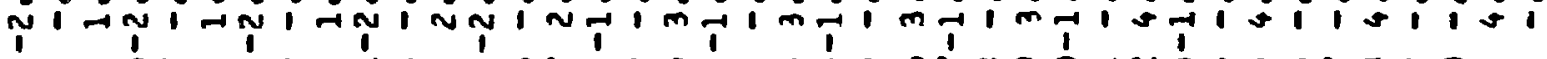

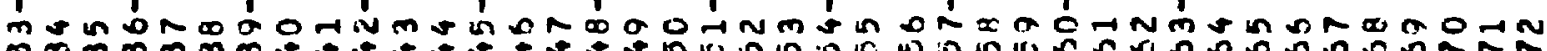

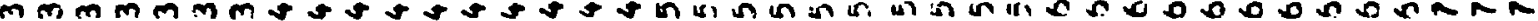




\begin{tabular}{|c|c|c|c|c|c|c|c|c|c|c|c|c|}
\hline $\begin{array}{l}0 \\
+9 \\
\dot{m} \\
\dot{m}\end{array}$ & $\stackrel{\infty}{m} \ddot{m}$ & $\begin{array}{l}a r \\
\sim \\
\ddot{m}\end{array}$ & $\begin{array}{l}0 \\
\dot{m} \\
\dot{m}\end{array}$ & $\begin{array}{ll}9 & m \\
0 & 0\end{array}$ & 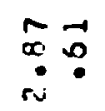 & $\underset{n}{R} R$ & $\begin{array}{l}\stackrel{P}{n} \\
\dot{n} \tilde{\sigma}\end{array}$ & $\begin{array}{ll}n & n \\
\sim & 0-1\end{array}$ & $\ddot{0} \stackrel{n}{m}$ & $\begin{array}{l}n \\
0 \\
0 \\
\dot{n}\end{array}$ & $\begin{array}{l}0 \\
0 \\
-\end{array}$ & $m \stackrel{5}{m}$ \\
\hline 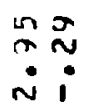 & $\begin{array}{ll}0 & -1 \\
0 & \overrightarrow{1} \\
\dot{N} & \bullet\end{array}$ & $\stackrel{n}{\sim} \tilde{0}$ & $\begin{array}{l}n: n \\
0: \\
\dot{v}\end{array}$ & $\begin{array}{l}\tilde{n} \\
\ddot{n}\end{array}$ & $\begin{array}{l}\vec{m} \\
\ddot{0}\end{array}$ & $\begin{array}{l}40 \\
\because \because\end{array}$ & $\begin{array}{l}\sigma \\
0 \\
-1\end{array}$ & $\stackrel{n}{\sim} \ddot{\square}$ & $\stackrel{0}{0} \stackrel{r}{m}$ & $\underset{\sim}{\stackrel{a}{n}}$ & 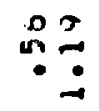 & $\cong ?$ \\
\hline 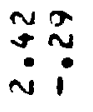 & $\begin{array}{l}m=1 \\
\ddot{n} \\
\dot{n}\end{array}$ & $\begin{array}{l} \pm 0 \\
\ddot{\sim}\end{array}$ & $\begin{array}{l} \pm n \\
\ddot{n} \\
\dot{n}\end{array}$ & $\begin{array}{l}\tilde{m} \\
\dot{n} \\
\dot{n}\end{array}$ & $\begin{array}{l}\infty \\
\infty \\
-1\end{array}$ & $\begin{array}{l}\overrightarrow{0} \\
\dot{-}\end{array}$ & $\stackrel{a}{n} \tilde{a}$ & $\stackrel{x}{\rightarrow} \stackrel{n}{\rightarrow} \ddot{\sim}$ & $\begin{array}{l}n \\
0 \\
0\end{array}$ & $\stackrel{n}{N} \cong$ & & $\begin{array}{l}\tilde{N} \\
\because \tilde{N}\end{array}$ \\
\hline 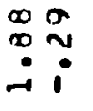 & $\begin{array}{l}0 \\
\propto \\
\bullet \\
-1\end{array}$ & $\begin{array}{l}N 0 \\
\dddot{N} \\
\ddot{0}\end{array}$ & $\begin{array}{l}m \\
n \\
-\end{array}$ & $\begin{array}{l}\text { is m } \\
\text { in ? } \\
-1\end{array}$ & $\vec{m} \stackrel{c}{a}$ & $\because 2$ & & & $\begin{array}{c}n \\
n \\
0\end{array}$ & mi & $\begin{array}{l}m \Omega \\
\because \ddot{0}\end{array}$ & \\
\hline \begin{tabular}{l}
$m$ \\
$m$ \\
\hdashline
\end{tabular} & $\begin{array}{l}\because 0 \\
\because i\end{array}$ & $\because \cong$ & $\stackrel{2 m}{\because}$ & $\begin{array}{ll}0 & n \\
0 & n \\
-1 & \end{array}$ & D? & $\begin{array}{ll}0 & 0 \\
0 & 0 \\
-1\end{array}$ & $\stackrel{m}{m}$ & 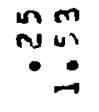 & $\underset{\sim}{\sim} \stackrel{n}{n}$ & $\begin{array}{l}10 \\
0\end{array}$ & :R & \\
\hline $\begin{array}{c}N \\
0 \\
0 \\
0 \\
m\end{array}$ & $\stackrel{n}{\sim} \underset{m}{\sim}$ & $\begin{array}{l}\infty \\
0 \\
0 \\
\ddot{n}\end{array}$ & $\stackrel{0}{0} \stackrel{0}{m}$ & 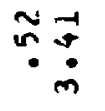 & $\stackrel{n}{m} \stackrel{n}{n}$ & $\because \stackrel{0}{?}$ & $\begin{array}{r}\vec{t} \cdot 0 \\
\dot{0} \\
\dot{m}\end{array}$ & $\begin{array}{l}9 \\
3 \\
i\end{array}$ & $\begin{array}{l}\because N \\
i m \\
i m\end{array}$ & $\stackrel{m}{n}$ & $\begin{array}{l}9 \\
9 \\
1\end{array}$ & \\
\hline$\because \tilde{N}$ & m & 足罢 & $? \approx$ & & $\overrightarrow{0}:$ & $\exists:$ & $\cong ّ$ & m: & & 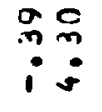 & $\begin{array}{l}92 \\
: \ddot{8} \\
i m\end{array}$ & \\
\hline $\begin{array}{ll}n & 0 \\
0 & 0 \\
0\end{array}$ & $\begin{array}{ll}N & 0 \\
0 & 0 \\
1 & 0\end{array}$ & $\begin{array}{l}05 \\
0 \text { in }\end{array}$ & $\begin{array}{l}\geq 8 \\
\because 8 \\
i \therefore\end{array}$ & $\begin{array}{l}0 r \\
\because r \\
i:\end{array}$ & $\begin{array}{l}\vec{n} \vec{a} \\
i \vec{s}\end{array}$ & $\because:$ & 00 & & & $\begin{array}{l}n \\
\vdots \\
i:\end{array}$ & $\begin{array}{l}3 \\
i m \\
i m\end{array}$ & \\
\hline $\begin{array}{ll}0 & -1 \\
-1 & -1 \\
1 & \text { in }\end{array}$ & $\begin{array}{l}m \\
\because n \\
i n\end{array}$ & $\stackrel{\infty}{\sim} \stackrel{n}{n}$ & $\begin{array}{l}\sim \\
m \sim \\
i \sim\end{array}$ & in & $\begin{array}{l}a \\
m \\
i:\end{array}$ & $\begin{array}{l}\simeq 5 \\
\vdots:\end{array}$ & $\begin{array}{l} \pm \infty \\
\pm: \\
i:\end{array}$ & $\begin{array}{l}0 \\
0 \\
:\end{array}$ & & $\begin{array}{l}a \\
0 \% \\
i=\end{array}$ & $\begin{array}{l}T= \\
i \stackrel{0}{n}\end{array}$ & $\begin{array}{l}\vec{c} \\
\dot{m}\end{array}$ \\
\hline $\begin{array}{l}\sim \\
m \\
i\end{array}$ & m & $\begin{array}{l}0 \\
0 \\
1 \\
1\end{array}$ & $\begin{array}{l} \pm 0 \\
\because \cong \\
\because \because\end{array}$ & $\begin{array}{l}\tilde{0} 0 \\
i \text { in }\end{array}$ & $\begin{array}{l}0 \pi \\
0 \\
i \\
0\end{array}$ & \begin{tabular}{l}
$\infty$ \\
\hdashline \\
$i$
\end{tabular} & $s$ & 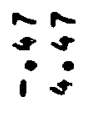 & & $\stackrel{\sim}{\because}:$ & $\begin{array}{l}n \\
\because \\
i\end{array}$ & $\because \tilde{R}$ \\
\hline $\begin{array}{l}\pi \\
\square \\
1\end{array}$ & $\begin{array}{ll}5 & 0 \\
: & 0\end{array}$ & $\begin{array}{l}0 \\
0 \\
5 \\
0\end{array}$ & $=\frac{\pi}{7}$ & $g$ ip & $\begin{array}{ll}9 & 0 \\
-? & 0 \\
1 & 0\end{array}$ & $\begin{array}{l}0 \\
7 \text { in } \\
i:\end{array}$ & $\begin{array}{ll}\infty & 5 \\
\mathfrak{m} & m\end{array}$ & $\begin{array}{ll}\infty & 0 \\
5 & 1\end{array}$ & $\begin{array}{l}n \\
\div \\
1\end{array}$ & $\vec{r} \vec{T}$ & $\begin{array}{l}\ddot{n} \\
\ddot{n} \dot{m}\end{array}$ & $\begin{array}{l}5 \\
i \geq\end{array}$ \\
\hline
\end{tabular}

ra

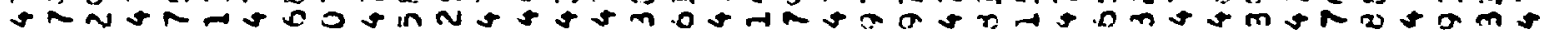

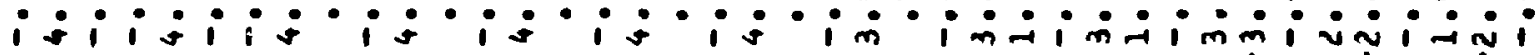
- IN

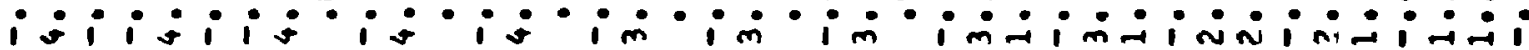

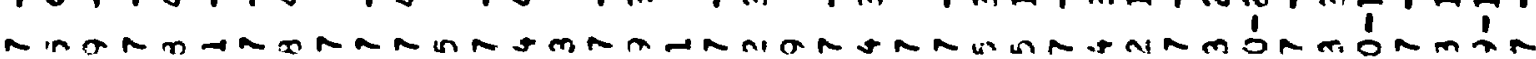

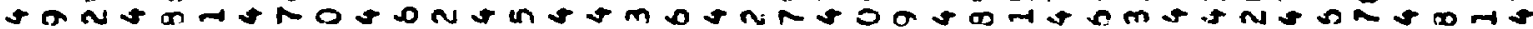

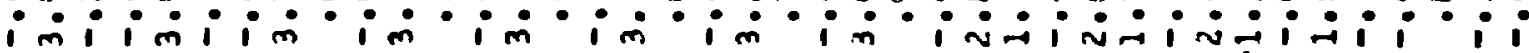

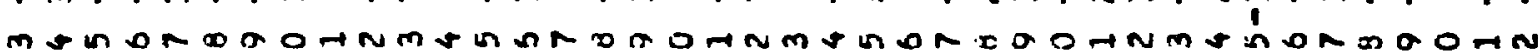
rRMNARR w

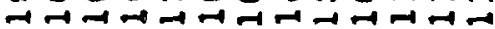

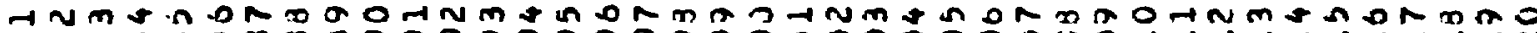

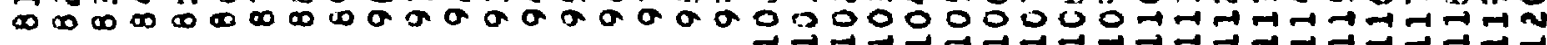




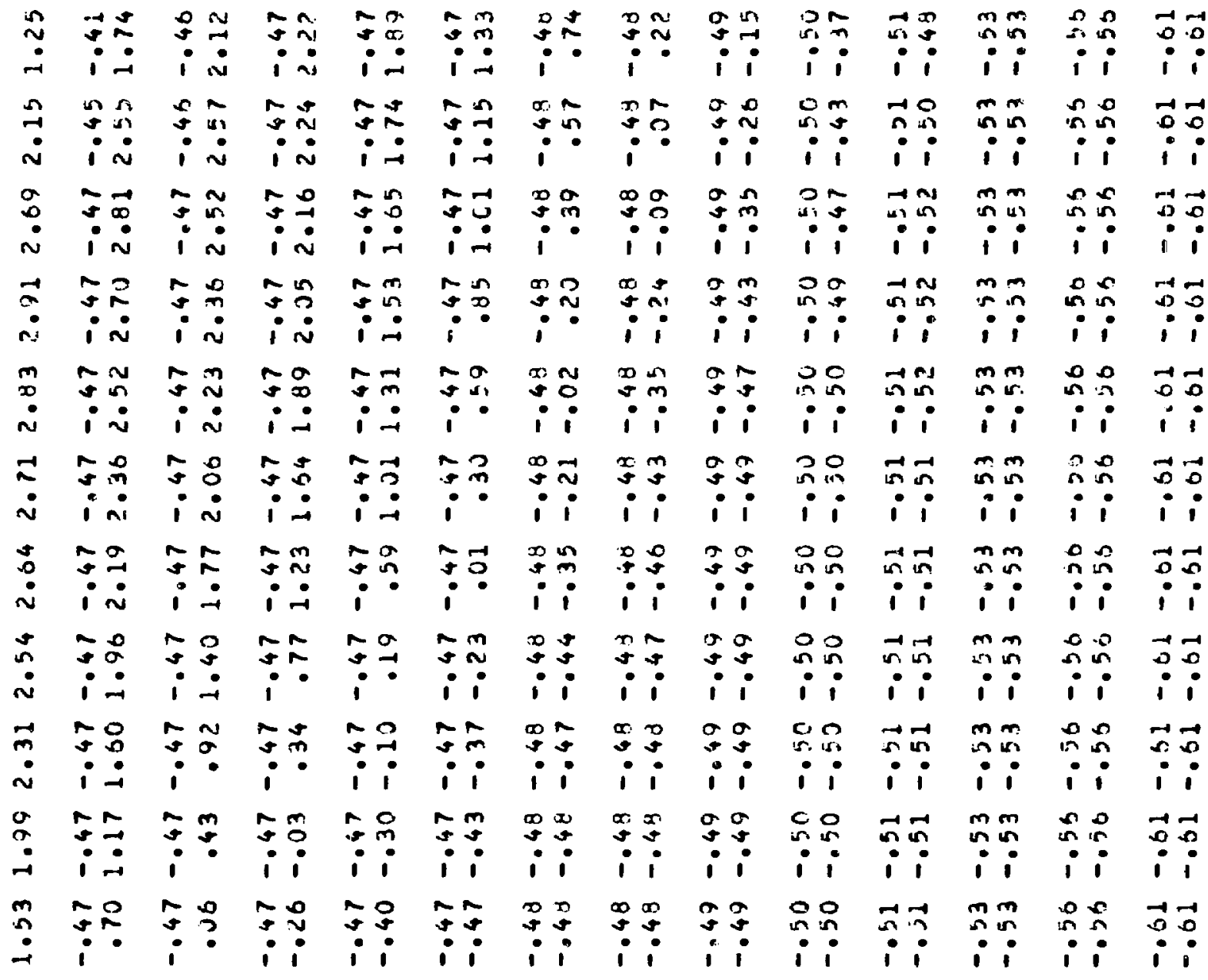

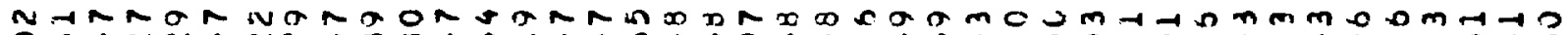
0 D T N N

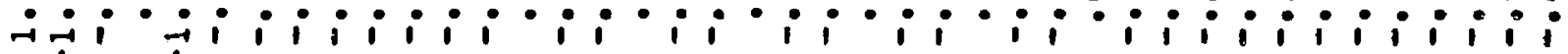
I

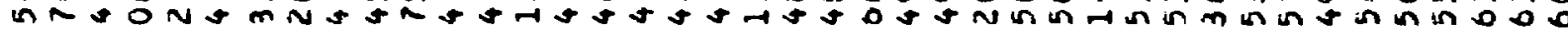

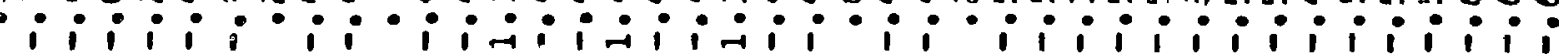

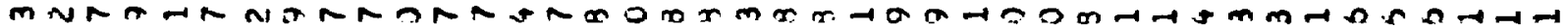

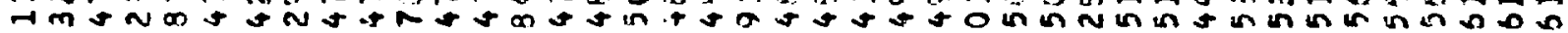

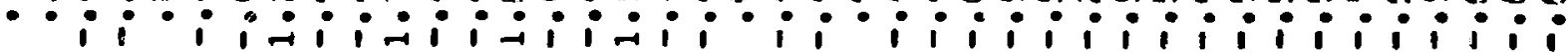

$m+n$ N

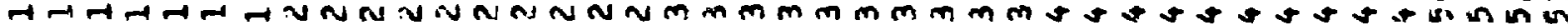
न्व

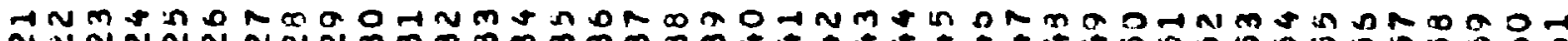

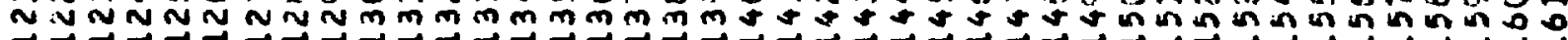

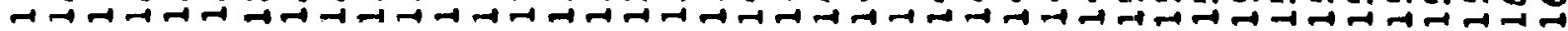

